草

Ramp-rate Sensitivity of SSC Dipole Magnet Prototypes

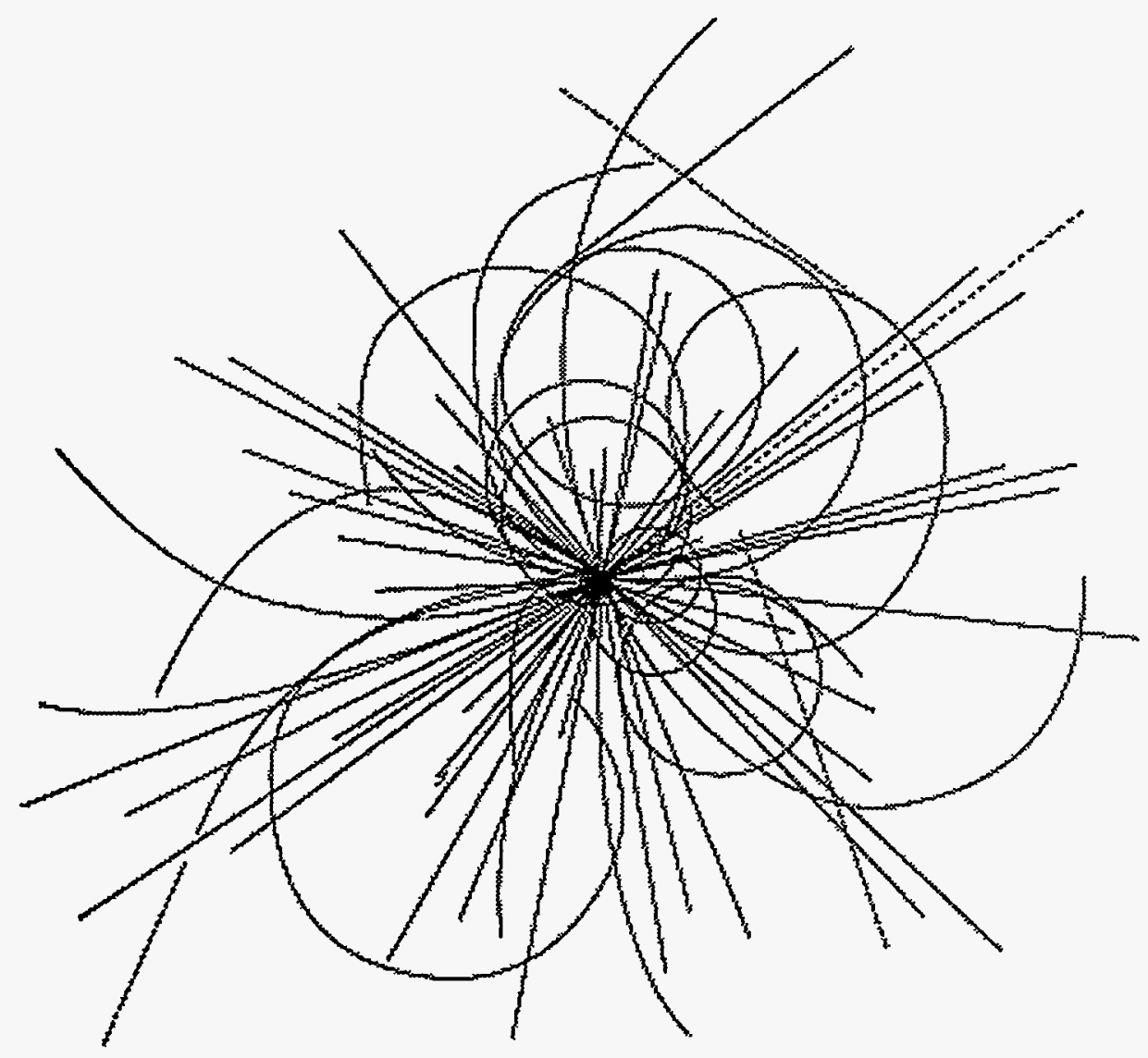

\section{Superconducting Super Collider Laboratory}

SSCL-Preprint-568

July 1994

Distribution Category: 414

A. Devred

T. Ogitsu
APPROVED FOR RELEASE OR PUBLIGETION-O.R. PATENTT GROUP BY.0.5. 


\section{Disclaimer Notice}

This report was prepared as an account of work sponsored by an agency of the United States Government. Neither the United States Government or any agency thereof, nor any of their employees, makes any warranty, express or implied, or assumes any legal liability or responsibility for the accuracy, completeness, or usefulness of any information, apparatus, product, or process diselosed, or represents that its use would not infringe privately owned rights. Relerence herein to any specific commercial product, process, or service by trade name, trademark. manulacturer, or otherwise, does not necessarily constitute or imply its endorsement, recommendation, or favoring by the United Stales Government or any agency thereof. The views and opinions of authors expressed herein do not necessarily state or reflect those of the United States Govemment or any agency thereol.

Superconducting Super Collider Laboratory is an equal opportunity employer. 


\section{DISCLAIMER}

Portions of this document may be illegible in electronic image products. Images are produced from the best available original document. 
SSCL-Preprint-568

\title{
Ramp-rate Sensitivity of SSC Dipole Magnet Prototypes*
}

\author{
A. Devred ${ }^{\dagger}$ and T. Ogitsu $\ddagger$ \\ Superconducting Super Collider Laboratory§ \\ 2550 Beckleymeade Ave. \\ Dallas, TX 75237
}

July 1994

\footnotetext{
"To be submitted to Particle Accelerators.

†Now at Centre d'Etudes de Saclay, 91191 Gif-sur-Yvette Cédex, France.

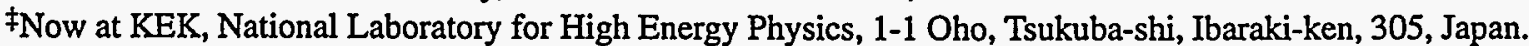

SOperated by the Universities Research Association, Inc., for the U.S. Department of Energy under Contract No. DE-AC35-89ER40486.
} 


\title{
RAMP-RATE SENSITIVITY OF \\ SSC DIPOLE MAGNET PROTOTYPES
}

\author{
A. DEVRED ${ }^{1}$ AND T. OGITSU2 \\ Superconducting Super Collider Laboratory* \\ 2550 Beckleymeade Avenue \\ Dallas, TX 75237, USA
}

\begin{abstract}
One of the major achievements of the magnet R\&D program for the Superconducting Super Collider (SSC) is the fabrication and test of a series of $205-\mathrm{cm}$ aperture, $15-\mathrm{m}$ long dipole magnet prototypes. The ramp rate sensitivity of these magnets appears to fall in at least two categories that can be correlated to the manufacturer and production batch of the strands used for the inner-coil cables. The first category, referred to as type- $A$, is characterized by a strong quench current degradation at high ramp rates, usually accompanied by large distortions of the multipole fields and large energy losses. The second category, referred to as type- $B$, is characterized by a sudden drop of quench current at low ramp rates, followed by a much milder degradation at larger rates. The multipole fields of the type-B magnets show little ramp-rate sensitivity, and the energy losses are smaller than for the type-A
\end{abstract}

1. Now at Centre d'Etudes de Saclay, 91191 Gif-sur-Yvette Cédex, France.

2. Now at KEK, National Laboratory for High Energy Physics, 1-1 Oho, Tsukuba-shi, Ibaraki-ken, 305, Japan.

* Operated by the Universities Research Association, Inc. for the U.S. Department of Energy under Contract No. DE-AC35-89ER40486.

$-1-$ 
magnets. The behavior of the Type-A magnets can be explained in terms of inter-strand eddy currents arising from low and non-uniform resistances at the crossovers between the strands of the two-layer Rutherford-type cable. Anomalies in the transport-current repartition among the cable strands are suggested as a possible cause for the type- $\mathrm{B}$ behavior. The origins of these anomalies have not yet been clearly identified. The SSC project was canceled by decision of the United States Congress on October 21, 1994.

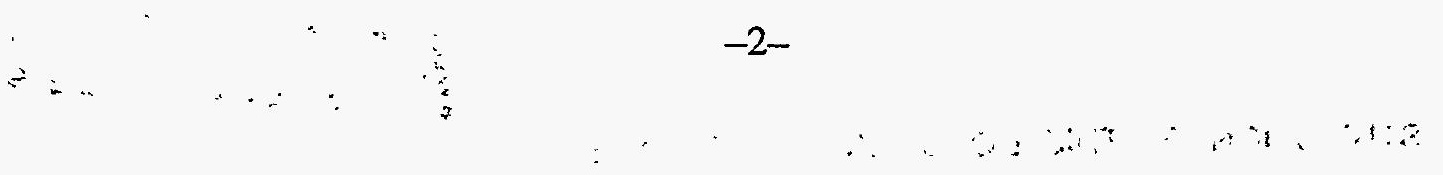


The SSC would have consisted of a chain of five accelerators, the last two of which relied on superconducting magnets. ${ }^{1}$ The penultimate stage, called the High Energy Booster (HEB), would have accelerated protons from $0.2 \mathrm{TeV}$ to $2.0 \mathrm{TeV}$ and would have served as an injector for the final stage, called the Collider. In the Collider, protons in two counterrotating rings would have been accelerated to $20 \mathrm{TeV}$ and stored for up to 24 hours while collisions occurred at several interaction points.

The design requirements for the HEB and Collider dipole magnets were very similar, save for the length ( $13 \mathrm{~m}$ for the HEB vs. $15 \mathrm{~m}$ for the Collider), the sagitta (18 $\mathrm{mm}$ for the HEB versus $1.8 \mathrm{~mm}$ for the Collider), and the current operating cycle. The injection time from the HEB to the Collider was fixed to 1 hour during which the HEB was to be cycled eight times between $-6650 \mathrm{~A}$ and $+6650 \mathrm{~A}$, at a ramp rate of $62 \mathrm{~A} / \mathrm{s}$. At the end of injection, the Collider was to be ramped from the injection current (of the order of $650 \mathrm{~A}$ ) to $6700 \mathrm{~A}$ at a ramp rate of $4 \mathrm{~A} / \mathrm{s}$. Hence, the HEB magnets were supposed to be operated in a bipolar mode and at a relatively high ramp rate, while the Collider magnets were supposed to be operated only in a monopolar mode and at a low ramp rate. The HEB would have required a little over 500 dipole magnets to be placed in a tunnel close to $11 \mathrm{~km}$ in circumference, while the Collider would have required more than 8000 dipole magnets, to be placed in a tunnel of the order of $87 \mathrm{~km}$ in circumference. The accelerator complex was to be built near the picturesque town of Waxahachie, 35 miles south of Dallas, Texas, USA.

An important step in the SSC magnet R\&D program was reached in 1992 with the completion of $205-\mathrm{cm}$ aperture, $15-\mathrm{m}$ long dipole magnet prototypes. Among these 
prototypes, aimed at both the Collider and the HEB, 7 were built and cold-tested at Brookhaven National Laboratory (BNL), while the 13 others were built and cold-tested at Fermi National Accelerator Laboratory (FNAL). The BNL magnets were designated as DCA207 through DCA213, while the FNAL magnets were designated as DCA311 through DCA323. These prototypes were also used as vehicles of technology transfer between the National Laboratories and the industrial contractors selected by the SSCL. Magnets DCA313 through DCA319 were assembled at FNAL by personnel from General Dynamics Space Systems (GDSS), while magnets DCA209 through DCA213 were assembled at BNL by personnel from Westinghouse Electric Company (WEC). GDSS was the main contractor for the Collider dipole magnets, while WEC was the main contractor for the HEB dipole magnets. Five of the industrially-assembled dipole magnets (magnets DCA313, DCA314, DCA315, DCA316, and DCA319), along with a 4-cm aperture, 5-m long quadrupole magnet and a spool piece, were used in an Accelerator System String Test (ASST) performed at the SSCL during the Summer of 1992. The magnet string reached the design current with no spontaneous quenches. ${ }^{2}$

After assembly completion, and prior to shipment to the SSCL, all the prototypes were individually cold-tested following similar run plans. The run plans typically called for two testing cycles separated by a warm-up to room temperature. Both cycles included quench testing and ramp rate sensitivity study as well as an extensive set of magnetic measurements. The magnetic measurements were performed either at constant current, to understand the field errors related to magnet geometry, or while ramping, to observe the dynamic field behavior during current cycles representative of the Collider or the HEB. It 
appeared that the quench performance at $4 \mathrm{~A} / \mathrm{s}^{3-7}$ and the geometric field errors ${ }^{8-12}$ were quite satisfactory; demonstrating, along with the ASST, the feasibility of the Collider. However, it also appeared that most of the prototypes exhibited a strong ramp rate sensitivity, resulting in a severe degradation of their quench current at $62 \mathrm{~A} / \mathrm{s}$, as well as sometimes very large field distortions while ramping. ${ }^{13,14}$ These unexpected dynamic behaviors raised some questions about the feasibility of the HEB and an aggressive program was undertaken, in cooperation with WEC, to determine their origins and find possible cures. The ramp-rate sensitivity program included the retest at BNL of a number of prototypes (magnets DCA312, DCA317, and DCA318), in-depth investigations of cable properties and manufacturing processes, and the fabrication of a series of short model magnets by WEC. 15

In this paper, after recalling the salient features of the BNL and FNAL 5-cm aperture, 15-m long dipole magnet prototypes, we review their ramp-rate sensitivity data, and we present some of the analyses that were carried out to interpret them.

\section{DESIGN AND FABRICATION}

The BNL and FNAL magnets use the same magnetic design 16 and rely on similar concepts for their mechanical design. ${ }^{3,17}$ Figure 1(a) displays a cross-sectional view of the BNL cold mass, while Fig. 1(b) displays a cross-sectional of the FNAL cold mass. The field of $6.6 \mathrm{~T}$ at $6500 \mathrm{~A}$ is produced by four racetrack-type coils that in their long straight sections approximate a two-layer cosine-theta distribution of conductors. The two inner coils (referred to as lower inner and upper inner) contain 19 turns and 3 copper wedges, while 


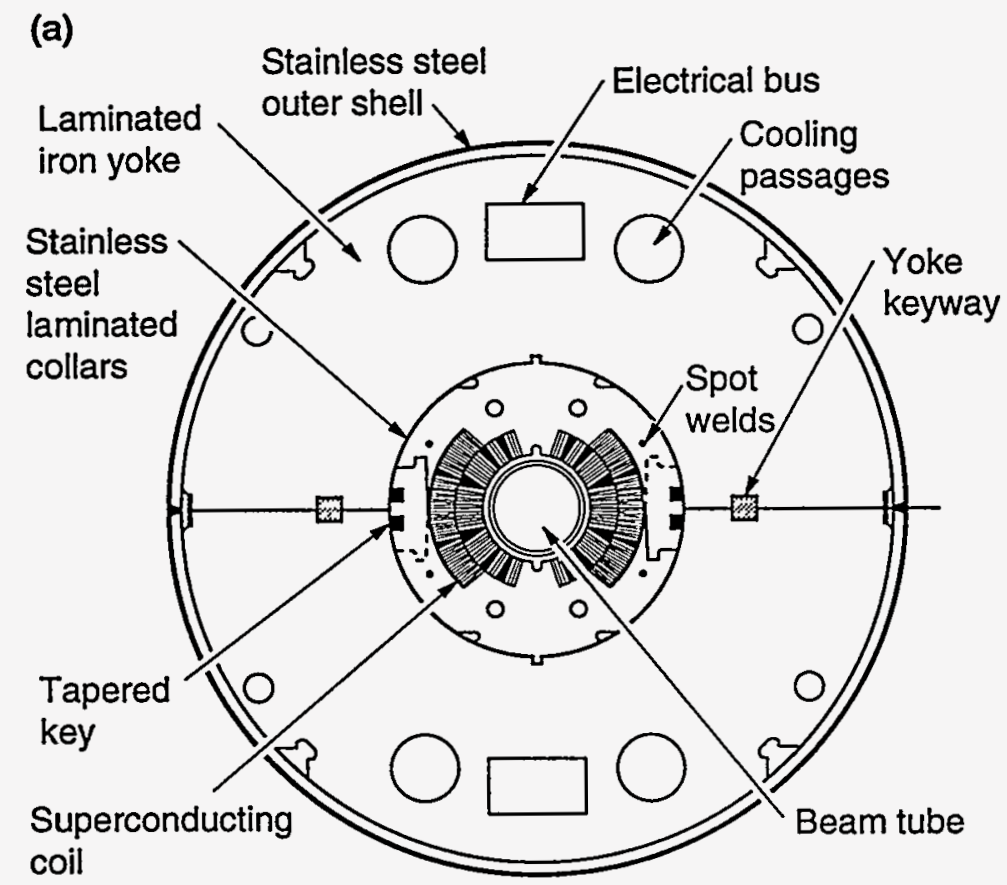

(b)

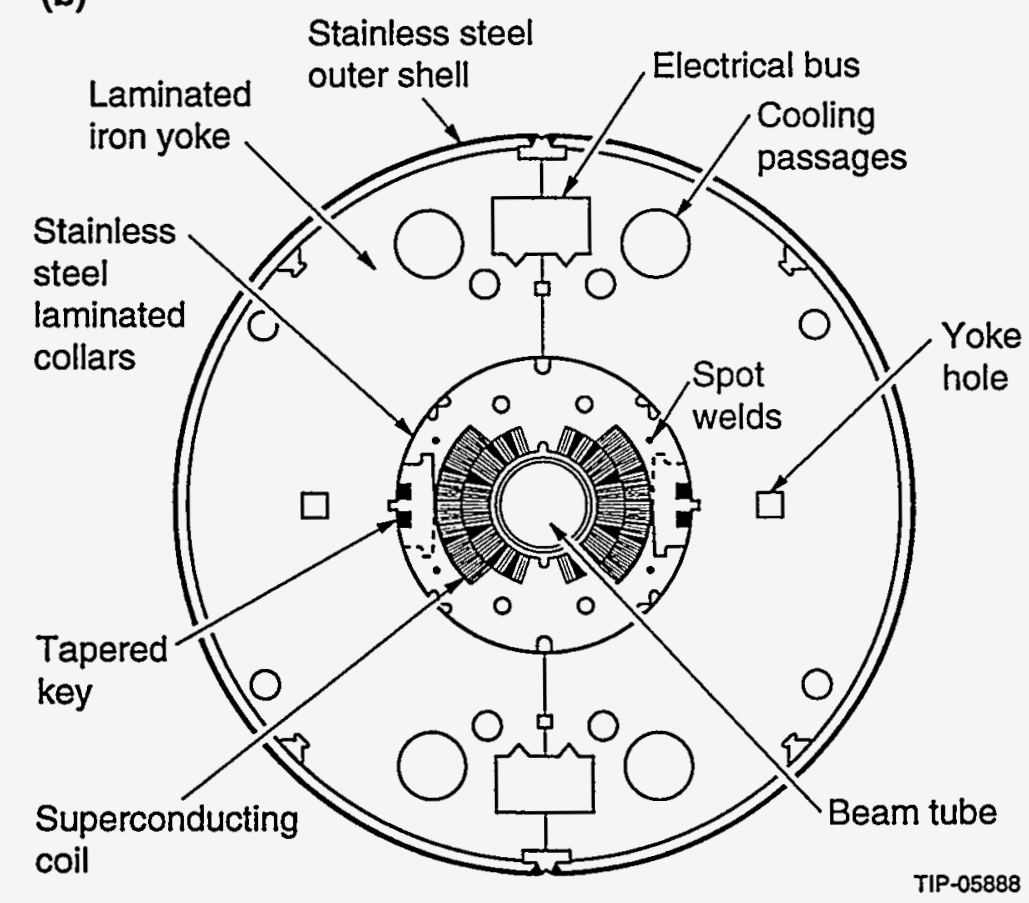

FIGURE 1: Cross-sectional view of the cold mass of 5-cm aperture SSC dipole magnet prototypes: a) BNL design, and b) FNAL design. 
the two outer coils (referred to as lower outer and upper outer) contain 26 turns and 1 copper wedge. The coils are mechanically restrained by means of laminated stainless steel collars that are designed to provide a large azimuthal pre-compression in order to compensate for the effects of the azimuthal component of the Lorentz force. ${ }^{18}$ The collared-coil assembly is encased in a laminated iron yoke around which a stainless steel outer shell is welded. The iron yoke provides a return path for the magnetic flux and enhances the field by about $20 \%$. The outer shell delimits the region of circulation for the 4.35-K, 0.4-MPa forced flow of supercritical helium. Yoke and shell are designed to tightly clamp the collared-coil assembly in order to stiffen the support against the radial and axial components of the Lorentz force.

The main difference between the BNL and FNAL mechanical designs is in the way the clamping of the collared-coil assembly by the yoke is realized. In the BNL design, the yoke is split horizontally and the clamping results from a positive interference along the vertical diameter. In the FNAL design, the yoke is split vertically, and the collar-yoke interference is along the horizontal diameter. Other specific features of the BNL design include internal splices between the conductors of the inner and outer coils, located at the radius of the outer coils and the fact that the coil ends are supported radially by collars similar to that of the magnet body. In the FNAL design, the splices are made at a radius larger than that of the outer coils and the coil ends are supported by a four-piece G10 collet that is compressed radially by a tapered aluminum cylinder. In both designs, the coil ends are loaded axially by four screws mounted into a thick stainless steel end plate that is welded to the outer shell. The BNL magnets also involved an improved cooling scheme, 
known as cross-flow cooling, 19 that involves the radial circulation of helium at set intervals along the magnet length from the yoke cooling passage to the coil cooling passage. Crossflow cooling was not implemented on the FNAL magnets.

Both inner and outer coils are wound from flat, two-layer, slightly keystoned, Rutherford-type cables. ${ }^{20}$ The inner-coil cable is made of 30 strands (strand diameter $0.808 \mathrm{~mm}$ ). It has a radial width of $12.34 \mathrm{~mm}$ and a mid-thickness of $1.458 \mathrm{~mm}$. Its keystone angle is $1.2^{\circ}$ and its pitch length is $86 \mathrm{~mm}$. The outer-coil cable is made of 36 strands (strand diameter $0.648 \mathrm{~mm}$ ). It has a radial width of $11.68 \mathrm{~mm}$ and a midthickness of $1.156 \mathrm{~mm}$. Its keystone angle is $1.05^{\circ}$ and its pitch length is $94 \mathrm{~mm}$. The strands themselves consist of an inner core and an outer sheath of copper sandwiching an annular multifilamentary composite. The inner core cross-sectional area is no more than $10 \%$ of the strand cross sectional area. The annular composite is made of thousands of $\mathrm{Nb}-46.5 \mathrm{wt} \%$ Ti filaments (filament diameter $6 \mu \mathrm{m}$ ), twisted together (twist pitch $13 \mathrm{~mm}$ ), and embedded in a copper matrix (interfilament spacing $1 \mu \mathrm{m}$ ). Each filament is surrounded by a niobium barrier to prevent the formation of copper-titanium intermetallic compound. ${ }^{21}$ The inner (outer) strands have a copper-to-superconductor ratio of 1.5 to 1 (1.8 to 1), except for the inner-coil cables of magnets DCA208, DCA320, and DCA321, which have a ratio of 1.3 to 1 .

The inner strands were purchased from three different manufacturers and were cabled on the same machine without mixing their origins. The same is true for the outer strands which were purchased from four different manufacturers. The critical current of the inner cables at $4.2 \mathrm{~K}$ and $7.0 \mathrm{~T}$ and that of the outer cables at $4.22 \mathrm{~K}$ and $5.6 \mathrm{~T}$ were measured to 
be in excess of 10000 A. 22 The strand manufacturers are reported to have used a copper with a Residual Resistivity Ratio (RRR) of the order of $300 .{ }^{23}$ (The RRR is defined as the ratio of the resistivity at $273 \mathrm{~K}$ and $0 \mathrm{~T}$ to the resistivity at $10 \mathrm{~K}$ and $0 \mathrm{~T}$ ). However, due to heavy cold-work during the operations of extrusion, drawing, and cabling, and the absence of an annealing step on the final strand or cable, the RRR of the as-received cable was measured to be 35 to 40 . No coating was applied on the strand surface, the state of which was only determined by the layer of copper oxide that developed during the various manufacturing steps. Tabs. I(a) through I(c) summarize the salient parameters of the cables used for the inner coils of the BNL and FNAL prototypes.

The bulk of the 20 prototypes (BNL magnets DCA207 through DCA211, and FNAL magnets DCA311 through DCA319) use a cable insulation consisting of a $0.025 \mathrm{~mm} \times$ $9.5 \mathrm{~mm}$ layer of $\mathrm{Kapton}^{\circledR}$ type-H film helically wrapped with a $50 \%$ overlap, completed by a $0.1 \mathrm{~mm} \times 9.5 \mathrm{~mm}$ layer of glass tape impregnated with B-stage epoxy wrapped with a 0.5-mm gap. Upon winding completion, the coils are heated under pressure to a temperature of $135^{\circ} \mathrm{C}$ for 90 minutes in order to cure the epoxy. Four of the remaining prototypes rely on a so-called all-Kapton ${ }^{\circledR}$ insulation scheme with either a polyimide adhesive coating (BNL magnets DCA212 and DCA213) or a B-stage epoxy coating (FNAL magnets DCA320 and DCA321). ${ }^{24}$ The last two FNAL prototypes (DCA322 and DCA323) also avoid the use of glass tape but rely on alternate materials to replace Kapton ${ }^{\circledR}$ and epoxy. The polyimide adhesive coating used in BNL magnets DCA212 and DCA213 required a short step at $225^{\circ} \mathrm{C}$ in the cure cycle, while the coils of FNAL magnets DCA320 through DCA323 were cured at $170^{\circ} \mathrm{C}$. Curing was always accompanied by a strong 
TABLE I(a): Salient parameters of the inner coil cables used in 5-cm aperture, 15-m long SSC/BNL dipole magnet prototypes with standard Kapton ${ }^{\circledR} /$ glass-tape insulation.

\begin{tabular}{|c|c|c|c|c|c|c|}
\hline $\begin{array}{c}\text { Magnet Name } \\
\text { and Coil }\end{array}$ & Cable Id & $\begin{array}{c}\text { Billet } \\
\text { Number }\end{array}$ & $\mathrm{Cu}: \mathrm{NbTi}$ & $\begin{array}{l}I_{\mathrm{c}} \text { at } 4.22 \mathrm{~K} \\
\text { and } 7 \mathrm{~T}(\mathrm{~A})^{\mathrm{a}}\end{array}$ & $\begin{array}{l}\text { Cable } \\
\text { RRR }^{a}\end{array}$ & $\begin{array}{r}\text { Coil } \\
\text { RRR }^{b}\end{array}$ \\
\hline DCA207 Upper & SSC-3-I-00054 & $819-821$ & 1.55 & 10904 & 39 & 220 \\
\hline DCA207 Lower & SSC-3-I-00055 & $819-822$ & 1.54 & 10836 & 38 & 222 \\
\hline DCA208 Upper & SSC-3-I-00067 & 1096 & 1.34 & 11371 & 37 & 133 \\
\hline DCA208 Lower & & & " & $"$ & 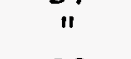 & 132 \\
\hline $\begin{array}{l}\text { DCA209 Upper } \\
\text { DCA209 Lower }\end{array}$ & SSC-3-O-O & $\underset{11}{2594-7}$ & 1.50 & 11265 & $\begin{array}{c}38 \\
" 1\end{array}$ & \\
\hline DCA210 Upper & SSC-3-0-00046 & $2596-7$ & 1.49 & 11331 & 38 & 196 \\
\hline DCA210 Lower & SSC-3-O-00045 & $2596-7$ & 1.48 & 11224 & 38 & 193 \\
\hline DCA211 Upper & SSC-3-O-00040 & $2593-5$ & 1.53 & 10873 & 40 & 189 \\
\hline DCA211 Lower & SSC-3-O-00050 & $2593-7$ & 1.48 & 11283 & 38 & 177 \\
\hline
\end{tabular}

a Measured on cable short sample.

$\mathrm{b}$ Measured on magnet during cold-testing. 
TABLE I(b): Salient parameters of the inner coil cables used for in 5-cm aperture, 15-m long SSC/FNAL dipole magnet prototypes with standard Kapton ${ }^{\circledR /}$ glass-tape insulation.

\begin{tabular}{|c|c|c|c|c|c|c|}
\hline $\begin{array}{c}\text { Magnet Name } \\
\text { and Coil }\end{array}$ & Cable Id & $\begin{array}{c}\text { Billet } \\
\text { Number }\end{array}$ & $\mathrm{Cu}: \mathrm{NbTi}$ & $\begin{array}{l}I_{\mathrm{c}} \text { at } 4.22 \mathrm{~K} \\
\text { and } 7 \mathrm{~T}(\mathrm{~A})^{\mathrm{a}}\end{array}$ & $\begin{array}{l}\text { Cable } \\
\text { RRR }^{a}\end{array}$ & $\begin{array}{c}\text { Coil } \\
\text { RRR }^{b}\end{array}$ \\
\hline $\begin{array}{l}\text { DCA311 Upper } \\
\text { DCA311 Lower }\end{array}$ & $\underset{n}{\mathrm{SSC}-3-\mathrm{S}-0023}$ & $\underset{11}{2605}$ & 1.51 & ${ }^{10079}$ & 37 & $\begin{array}{l}175 \\
175\end{array}$ \\
\hline DCA312 Upper & SSC-3-I-00047 & $819-822$ & 1.53 & 10764 & 40 & 167 \\
\hline DCA312 Lower & SSC-3-I-00035 & 642 & 1.53 & 10673 & 42 & 165 \\
\hline $\begin{array}{l}\text { DCA313 Lower } \\
\text { DCA313 Upper }\end{array}$ & SSC-3-I-I-00052 & $\underset{11}{822 / 857}$ & 1.52 & 10512 & 42 & $\begin{array}{l}170 \\
173\end{array}$ \\
\hline DCA314 Upper & SSC-3-I-00052 & $822 / 857$ & 1.52 & 10512 & 42 & 174 \\
\hline DCA314 Lower & SSC-3-I-00049 & $819-822$ & 1.52 & 10869 & 39 & 171 \\
\hline DCA315 Upper & SSC-3-I- -00049 & $819-822$ & 1.52 & 10869 & 39 & 162 \\
\hline $\begin{array}{l}\text { DCA315 Lower } \\
\text { DCA316 Upper }\end{array}$ & SSC-3-O-00034 & 2593 & & & & 177 \\
\hline DCA316 Lower & SSC-3-O-00037 & $\begin{array}{c}2593 \\
2593-4\end{array}$ & $\begin{array}{l}1.21 \\
1.55\end{array}$ & $\begin{array}{l}11085 \\
10985\end{array}$ & $\begin{array}{l}39 \\
39\end{array}$ & $\begin{array}{l}67 \\
70\end{array}$ \\
\hline DCA317 Upper & SSC-3-I-00052 & $822 / 857$ & 1.52 & 10512 & 42 & $\begin{array}{c}78 \\
157\end{array}$ \\
\hline DCA317 Lower & SSC-3-I-00049 & $819-822$ & 1.52 & 10869 & 39 & 163 \\
\hline DCA318 Upper & SSC-3-O-00038 & $2593-5$ & 1.54 & 10851 & 40 & 102 \\
\hline $\begin{array}{l}\text { DCA318 Lower } \\
\text { DCA319 Inner }\end{array}$ & & & & & & 105 \\
\hline $\begin{array}{l}\text { DCA319 Upper } \\
\text { DCA319 Lower }\end{array}$ & $\begin{array}{l}\text { SSC-3-O-00043 } \\
\text { SSC-3-O-00049 }\end{array}$ & $2594-7$ & 1.51 & 11038 & 39 & 105 \\
\hline DCA319 Lower & SSC-3-0-00049 & $2594-7$ & 1.50 & 11210 & 37 & 108 \\
\hline
\end{tabular}

\footnotetext{
${ }^{a}$ Measured on cable short sample.

b Measured on magnet during cold-testing.
} 
TABLE I(c): Salient parameters of the inner coil cables used in 5-cm aperture, 15-m long SSC dipole magnet prototypes with all-polyimide insulation.

\begin{tabular}{|c|c|c|c|c|c|c|}
\hline $\begin{array}{l}\text { Magnet Name } \\
\text { and Coil }\end{array}$ & Cable Id & $\begin{array}{c}\text { Billet } \\
\text { Number }\end{array}$ & $\mathrm{Cu}: \mathrm{NbTi}$ & $\begin{array}{l}I_{\mathrm{c}} \text { at } 4.22 \mathrm{~K} \\
\text { and } 7 \mathrm{~T}(\mathrm{~A})^{\mathrm{a}}\end{array}$ & $\begin{array}{l}\text { Cable } \\
\text { RRR }^{a}\end{array}$ & $\begin{array}{c}\text { Coil } \\
\text { RRR }^{b}\end{array}$ \\
\hline DCA212 Upper & SSC-3-S-00034 & $2735-2,3$ & 1.51 & 10368 & 35 & 137 \\
\hline DCA212 Lower & SSC-3-S-00033 & $2735-2,3$ & 1.51 & 10308 & 35 & 136 \\
\hline DCA213 Upper & SSC-3-S-00034 & $2735-2,3$ & 1.51 & 10368 & 35 & 139 \\
\hline DCA213 Lower & SSC-3-S-00040 & $2735-1,2,3$ & 1.54 & 10039 & 36 & 140 \\
\hline DCA320 Upper & SSC-3-I-00066 & 1095 & 1.37 & 11266 & 36 & 81 \\
\hline DCA320 Lower & SSC-3-I-00068 & 1097 & 1.34 & 11095 & 37 & 182 \\
\hline DCA321 Upper & SSC-3-S-00025 & $2444-1,2$ & 1.31 & 11101 & 39 & 143 \\
\hline DCA321 Lower & & & & & & 133 \\
\hline DCA322 Upper & SSC-3-S-00035 & $2735-1,3$ & 1.48 & 10452 & 34 & 66 \\
\hline DCA322 Lower & SSC-3-S-00036 & $2735-1,3$ & 1.48 & 10535 & 35 & 66 \\
\hline DCA323 Upper & SSC-3-S-00040 & $2735-1,2,3$ & 1.54 & 10039 & 36 & 61 \\
\hline DCA323 Lower & SSC-3-S-00046 & & 1.58 & 10216 & 36 & 62 \\
\hline
\end{tabular}

a Measured on cable short sample.

$b$ Measured on magnet during cold-testing. 
annealing of the conductor copper and the coil RRRs measured during magnet cold test were usually in excess of 100 (see Tab. I).

All the prototypes were instrumented with voltage taps on the turns the closest to the collar poles for quench start localization.

\section{QUENCH PERFORMANCE}

\subsection{Training}

Figure 2(a) presents a summary plot of the quench performance at $4.35 \mathrm{~K}$ of the BNL prototypes, while Fig. 2(b) presents a similar plot for the FNAL prototypes. For each magnet, the data correspond to the first ramps to quench after the first two cooldowns, and the dashed line marks the thermal cycle to room temperature. Detailed discussions of these data can be found elsewhere. ${ }^{3-7}$ At BNL, magnet DCA213 went directly to plateau, while the other magnets exhibited one or two training quenches. The training quench currents were all above $7100 \mathrm{~A}$, except for the first quench of magnet DCA211, which occurred at 6692 A. Three of the FNAL magnets (magnets DCA313, DCA314, and DCA317) exhibited a training quench below $6000 \mathrm{~A}$. These three quenches were localized in the same area, and are attributed to a discrepancy in the assembly of a two-piece G10 key supporting the turnaround of the inner-coil pole turn from the inside. After the first quench, the three magnets went above $7200 \mathrm{~A}$ and the problem did not resurface. With the exception of magnet DCA316, which had its first quench at $6410 \mathrm{~A}$, the remaining FNAL magnets with the standard Kapton ${ }^{\circledR} /$ glass-tape insulation (magnets DCA311, DCA312, DCA315, DCA318, and DCA319) reached a current near their expected conductor limit on the first quench. (The second quench of magnet DCA319, which occurred at $6415 \mathrm{~A}$ while ramping 
at $16 \mathrm{~A} / \mathrm{s}$, is considered as ramp-rate related.) The four magnets with the all-polyimide insulation (magnets DCA320 through DCA323) that were built while the SSC magnet program at FNAL was being shut down exhibited poorer quench performance than their predecessors. All the magnets reached a stable plateau at $4.35 \mathrm{~K}$, and the plateau quenches were all localized in the inner coil pole turn where the magnetic field is the largest.

(a)

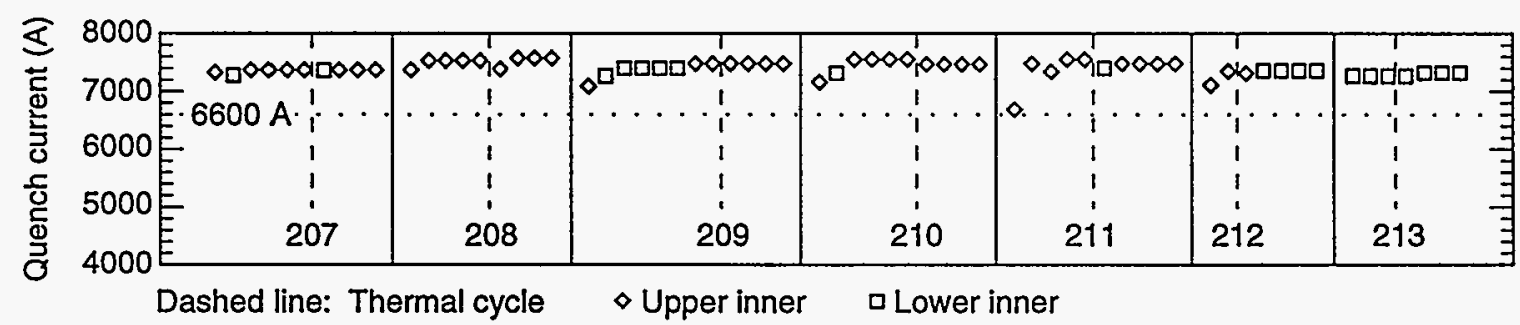

(b)

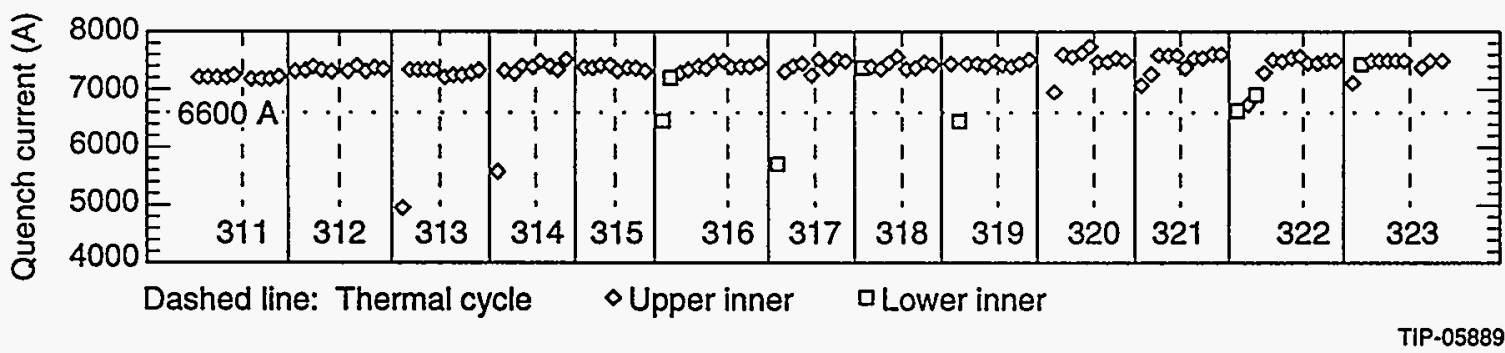

FIGURE 2: Quench performance at $4.35 \mathrm{~K}$ of $5-\mathrm{cm}$ aperture, $15-\mathrm{m}$ long SSC dipole magnet prototypes at $4.35 \mathrm{~K}$ : a) BNL magnets, b) FNAL magnets. For each magnet, the data correspond to the first ramps to quench after the first two cooldowns, and the dashed line marks the thermal cycle to room temperature.

\subsection{Ramp rate sensitivity}

Once the quench plateau is established, the test plan calls for a series of quenches at increasing ramp rates. Figures 3(a) and 3(b) display summary plots of quench current versus ramp rate for selected BNL and FNAL prototypes. The selection was made 
(a)

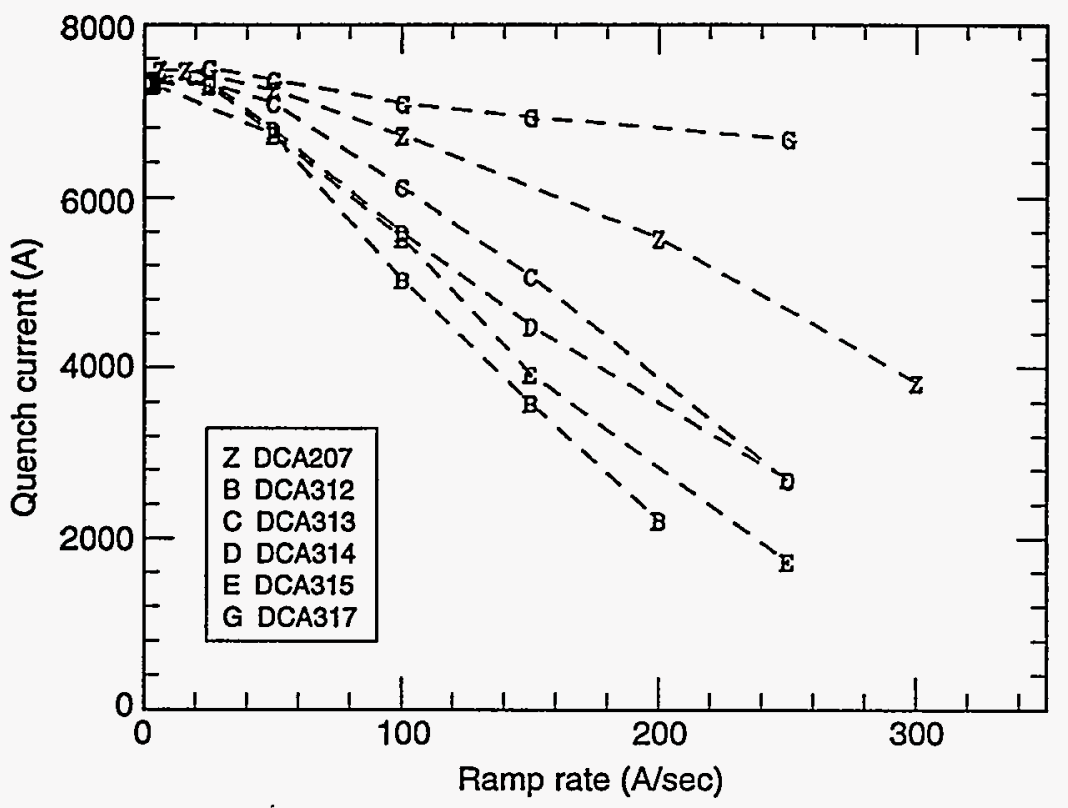

(b)

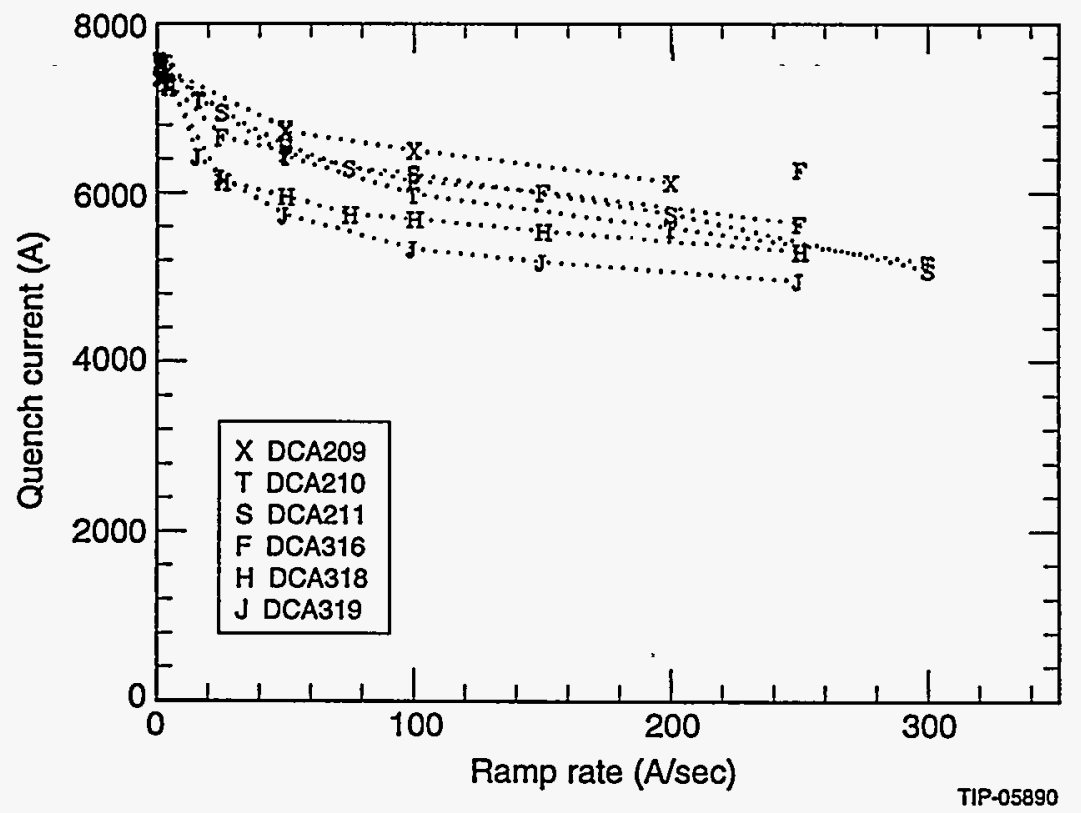

FIGURE 3: Ramp rate sensitivity of selected 5-cm aperture, 15-m long SSC dipole magnet prototypes. The magnets are grouped according to the manufacturer and the production batch of their inner cable strands: a) magnets with IGC inner strands (800-series billets), and b) magnets with OST inner strands (2500-series billets). 
according to the manufacturer and the production batch of the inner cable strands. With the exception of the lower inner coil of magnet DCA312, the inner cable strands of the Fig. 3(a) magnets were manufactured by Intermagnetics General Corporation (IGC) from 800-series billets (see Tab. I). The inner cable strands of the Fig. 3(b) magnets were manufactured by Oxford Superconducting Technology (OST) from 2500-series billets. Note that all the magnets in Figs. 3(a) and 3(b) rely on the standard Kapton®/glass-tape insulation with a curing temperature of $135^{\circ} \mathrm{C}$. The magnets not included in Fig. 3 use inner cable strands from disparate origins and/or rely on alternate insulation schemes with disparate curing cycles.

It appears that, for the magnets of Fig. 3(a), the quench current remains roughly constant for ramp rates up to $25 \mathrm{~A} / \mathrm{s}$, above which it starts to decrease linearly as a function of ramp rate. The worst case is magnet DCA312, which at $200 \mathrm{~A} / \mathrm{s}$, quenches at $2180 \mathrm{~A}$, corresponding to about $30 \%$ of its initial quench current. In comparison, the behavior of the magnets in Fig. 3(b) is quite different. The quench current starts by dropping significantly at low ramp rates, while the degradation at large ramp rates is much milder. The worst case is magnet DCA319, for which the quench current decreases from $7334 \mathrm{~A}$ at $1 \mathrm{~A} / \mathrm{s}$ to $6156 \mathrm{~A} / \mathrm{s}$ at $25 \mathrm{~A} / \mathrm{s}$, but is still of the order of $5000 \mathrm{~A}$ at $250 \mathrm{~A} / \mathrm{s}$. The quench behavior of the Fig. 3(a) magnets is referred to as type-A, while that of the Fig. 3(b) magnets is referred to as type-B.

As mentioned in the introduction, three of the magnets initially tested at FNAL (magnets DCA312, DC317, and DCA318) were retested at BNL. The BNL and FNAL test facilities are quite similar except for the magnet end from which the helium is fed and for 
the helium mass-flow rate. At BNL, the helium is fed from the magnet lead end (LE), where the current leads are located, while, at FNAL, it is fed from the opposite end, called the non-lead end (NLE). Typical helium mass flow rates are 40 to $50 \mathrm{~g} / \mathrm{s}$ at FNAL and 140 to $150 \mathrm{~g} / \mathrm{s}$ at BNL. Figure 4 presents a comparison of the ramp-rate sensitivity data taken at BNL and FNAL. Here, the quench currents have been normalized to the average plateau quench current of the given magnet at the given temperature. Looking first at the 4.35- $\mathrm{K}$ data, it appears that, despite the difference in helium mass flow rate, the quench currents measured at BNL are similar to those measured at FNAL. (Note that the 25-A/s

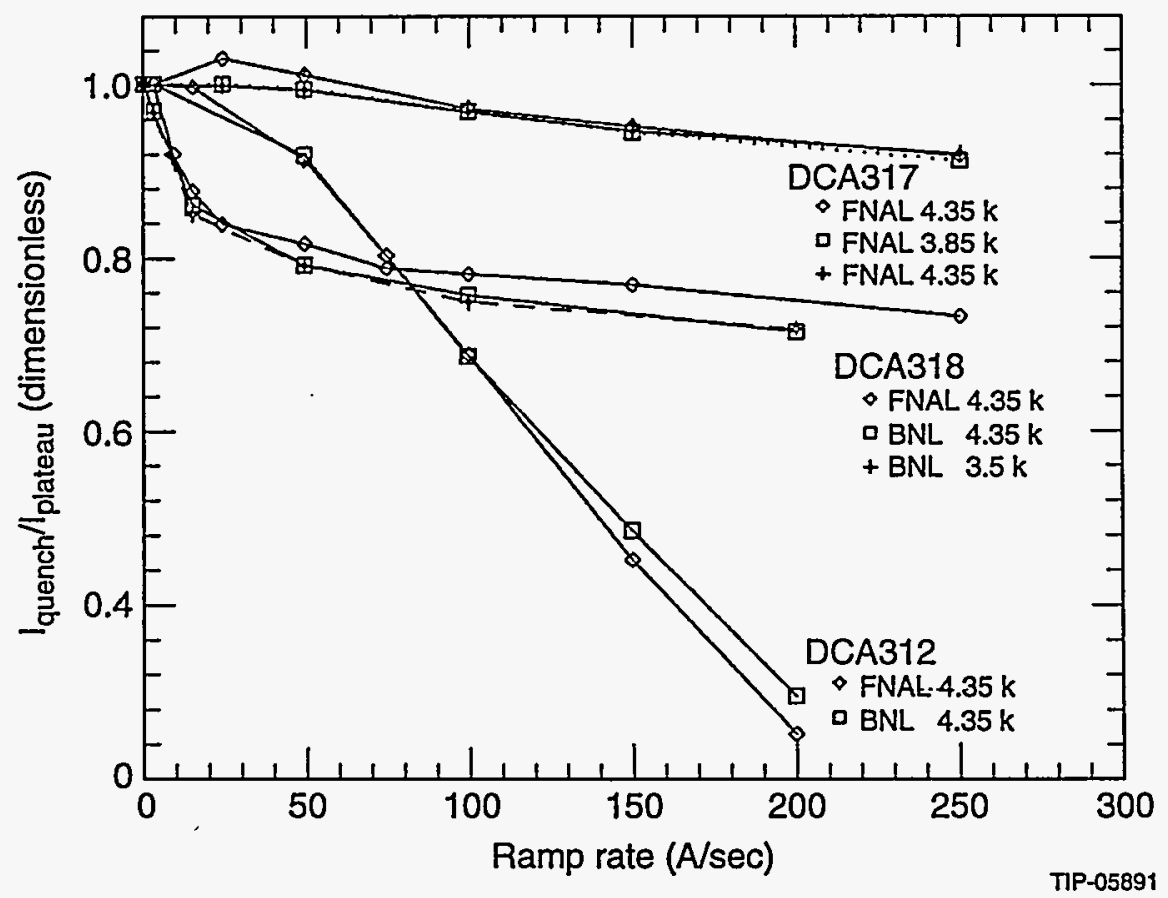

FIGURE 4: Comparison of the ramp rate sensitivity of 5-cm aperture, $15-\mathrm{m}$ long SSC dipole magnet prototypes between tests at $4.35 \mathrm{~K}$ at FNAL and at BNL (continuous lines), and tests at lower temperatures (dashed and dotted lines). The quench currents are normalized to the plateau quench current of the given magnet at the given temperature. 
and $50-\mathrm{A} / \mathrm{s}$ quenches of magnet DCA317 at FNAL were at a slightly lower temperature than the other 4.35-K nominal quenches.) Also displayed in Fig. 4 are some ramp-rate sensitivity data taken at lower temperatures $(3.85 \mathrm{~K}$ for magnet DCA317 at FNAL and $3.5 \mathrm{~K}$ for magnet DCA318 at BNL). It appears that, after normalization to the average plateau quench current at the given temperature, the lower-temperature data lay atop of the 4.35-K data.

\subsection{Localization of ramp-rate related quenches}

A first indication of the quench start localization is given by the voltage tap data. For all magnets, the lowest ramp-rate quenches are localized in the inner coil pole turn. For the type-A magnets, the quenches keep originating in the inner coil pole turn for ramp rates up to $25 \mathrm{~A} / \mathrm{s}$. For rates larger than $50 \mathrm{~A} / \mathrm{s}$, however, the quench origin shifts towards the inner coil midplane, between turn 1 and turn 13, where there are no voltage taps. (The turns are counted starting from the coil midplane; the inner coil pole turn is turn 19.) For the type-B magnets, a similar shift in quench start localization is observed, but it occurs much sooner-between $1 \mathrm{~A} / \mathrm{s}$ and $4 \mathrm{~A} / \mathrm{s}$ - and is concomitant with the sudden drop in quench current.

More accurate localizations of the high ramp-rate quenches can be obtained from the retest data of magnets DCA312, DCA317, and DCA318 at BNL. During these tests, a series of investigations were carried out using an array of stationary pickup coils inserted in the magnet bore. This array, which detects the field distortions resulting from quench development, was inspired by a technique recently developed by the European Organization for Nuclear Research (CERN), ${ }^{25}$ and is described elsewhere. ${ }^{26}$ The 
experiments were quite successful, and allowed determination of both the axial and azimuthal localizations of the quench start.

Table II summarizes the localizations of the $4.35-\mathrm{K}$ ramp-rate quenches of magnets DCA312, DCA317, and DCA318 estimated from the pickup coils' data. It was verified that the quench currents and the quench start localizations were very reproducible. It was also verified that the pickup coil array did not introduce any noticeable perturbations. (After introduction of the array, the bore tube was sealed and evacuated as for regular quench tests.) All the quenches listed in Tab. II originated in the inner coils. The coil quadrants are defined by facing the magnet from the non-lead end and are counted counter-clockwise starting from the top-right quadrant. For all three magnets, it appears that, at a given ramp rate, the quenches always originate at the same location but that the location varies from one ramp rate to the other. For instance, in the case of magnet DCA312, the $16 \mathrm{~A} / \mathrm{s}$ quenches originate in the inner coil pole turn close to the non-lead end. At $100 \mathrm{~A} / \mathrm{s}$, the quenches shift towards the coil midplane and the magnet lead end. At 150 and $200 \mathrm{~A} / \mathrm{s}$, however, the quenches are again localized towards the non-lead end. It appears also that, for the quenches originating in turns instrumented with voltage taps, the localizations estimated from the pickup coils' data are consistent with that estimated from the voltage taps' data. 
TABLE II: Estimated localizations of ramp-rate related quenches during the 4.35-K re-tests of magnets DCA312, DCA317, and DCA318 at BNL. (All quenches originated in the inner coils.)

\begin{tabular}{|c|c|c|c|c|}
\hline Magnet & Ramp & Quench & Axial Distance & Turn \\
\hline \multirow[t]{2}{*}{ Name } & Rate & Current & from Center & Number \\
\hline & $(\mathrm{A} / \mathrm{s})$ & (A) & (Direction) & (Quadrant) \\
\hline \multirow[t]{4}{*}{ DCA312 } & 16 & 7250 & $7 \mathrm{~m}$ (NLE) & $19(\mathrm{Q} 2)$ \\
\hline & 100 & 5000 & $5.5 \mathrm{~m}$ (LE) & 3 11 (Q1) \\
\hline & 150 & 3290 & $5.5 \mathrm{~m}$ (NLE) & $6 \sim 12(\mathrm{Q} 2)$ \\
\hline & 200 & 1860 & $4 \mathrm{~m}$ (NLE) & $6 \sim 10(\mathrm{Q} 2)$ \\
\hline \multirow[t]{5}{*}{ DCA317 } & 25 & 7100 & $2 \mathrm{~m}$ (NLE) & $19(\mathrm{Q} 1)$ \\
\hline & 50 & 7060 & $6.5 \mathrm{~m}$ (NLE) & 8 9 (Q2) \\
\hline & 100 & 6880 & $2 \mathrm{~m}$ (NLE) & $1 \sim 4(\mathrm{Q} 1)^{\mathrm{a}}$ \\
\hline & 150 & 6720 & $6.5 \mathrm{~m}$ (NL.E) & $1 \sim 4(\mathrm{Q} 1)^{\mathrm{a}}$ \\
\hline & 250 & 6700 & $3.5 \mathrm{~m}$ (NLE) & $1 \sim 4(\mathrm{Q} 1)^{\mathrm{a}}$ \\
\hline \multirow[t]{6}{*}{ DCA318 } & 1 & 7450 & $6 \mathrm{~m}$ (NLE) & $19(\mathrm{Q} 2)$ \\
\hline & 4 & 7230 & $6.5 \mathrm{~m}$ (NLE) & $1 \sim 5(\mathrm{Q} 4)$ \\
\hline & 16 & 6500 & $6 \mathrm{~m}$ (LE) & 5 9 (Q2) \\
\hline & 50 & 5900 & $5 \mathrm{~m}$ (LE) & 5 9 (Q2) \\
\hline & 100 & 5600 & $5.5 \mathrm{~m}$ (NLE) & 1 7 (Q1) \\
\hline & 200 & 5300 & $5 \mathrm{~m}$ (NLE) & $2 \sim 9(\mathrm{Q} 2)$ \\
\hline
\end{tabular}

a Voltage taps' data show that the first coil to go resistive is the lower inner coil (Q3/Q4), followed, within $10 \mathrm{~ms}$, by the upper inner coil (Q1/Q2). The localizations reported here are that derived from the pickup coils' data. 
During the tests of magnets DCA312, DCA317, and DCA318, a number of special ramps to quench were performed (all at $4.35 \mathrm{~K}$ ) in order to better qualify the mechanisms underlying the type- $\mathrm{A}$ and type-B behaviors.

In the following, $r_{0}$ designates a ramp rate, and $I_{\mathrm{q}}\left(r_{0}\right)$ designates the quench current at ro. As it appears that the quench current depends on the magnet excitation history, the test sequence is always: quench, special ramp to quench.

\subsection{Paused ramps}

The first series of ramps, referred to as paused ramps, are intended to measure the effect of a pause a few hundred amperes below the quench current. In a paused ramp, the magnet is first ramped at $r_{0}$ to a current of the order of $I_{\mathrm{q}}\left(r_{0}\right)$ minus $200 \mathrm{~A}$. The ramp is then paused for a duration, $\tau$, after which, the magnet is ramped to quench at $r_{0}$. The corresponding quench current is noted $I_{r_{0}}(\tau)$. During the pause, the magnet coil, which may have been heated during the ramp, is expected to cool off, resulting in an increase in temperature margin and, subsequently, in quench current.

Figure 5 presents a summary plot of quench current versus pause duration for a series of paused ramps on magnets DCA312 and DCA318 where $r_{0}=100 \mathrm{~A} / \mathrm{S}$. For magnet DCA312 the pause was at $4800 \mathrm{~A}$, while for magnet DCA318 it was at $5400 \mathrm{~A}$. The continuous and dashed lines of Fig. 5 correspond to a least-square fitting of the quench data by the function, $f_{\mathrm{r}_{0}}$, defined as

$$
f_{\mathrm{r}_{0}}(\tau)=I_{0}+I_{1}\left[1-\exp \left(-\frac{\tau}{\tau_{1}}\right)\right]+I_{2}\left[1-\exp \left(-\frac{\tau}{\tau_{2}}\right)\right]
$$


where the parameters $I_{0}, I_{1}, \tau_{1}$, and $\tau_{2}$, along with the $\chi^{2}$ of $\left(I_{\mathrm{r}_{0}}(\tau)-f_{\mathrm{r}_{0}}(\tau)\right)$ are given in Tab. III.

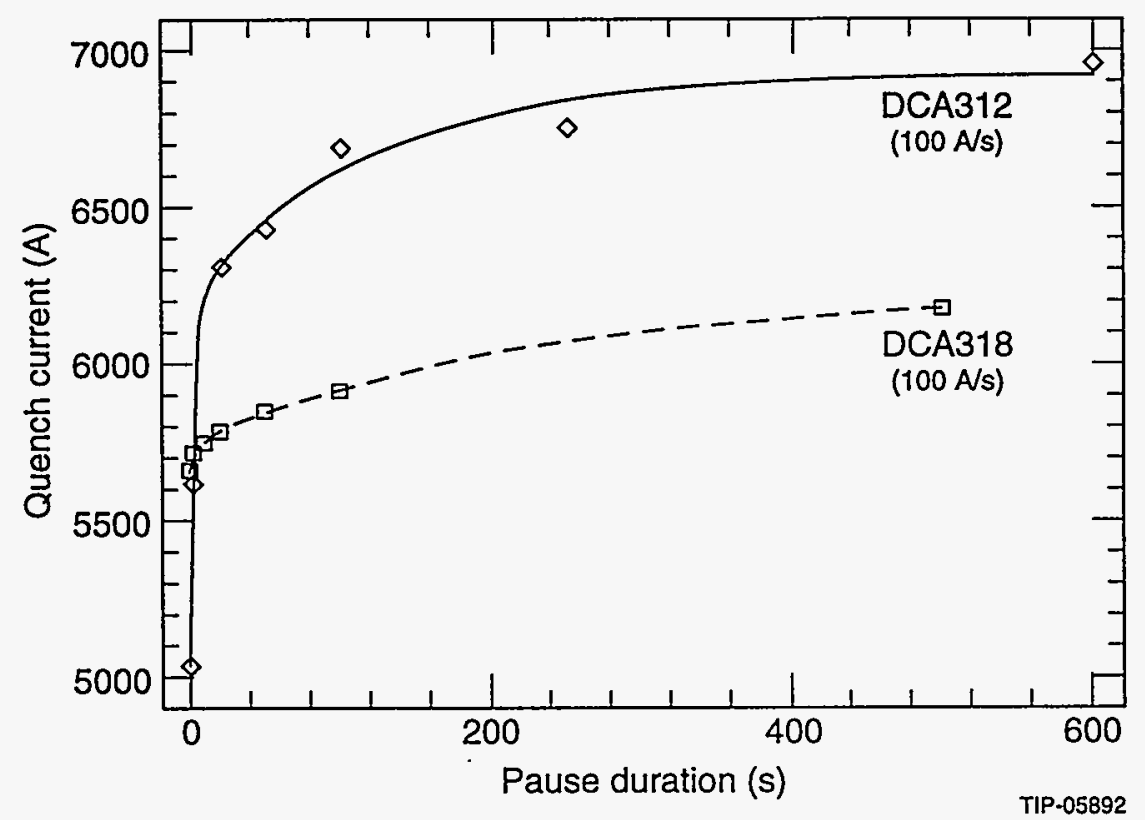

FIGURE 5: Quench current versus pause duration for a series of paused ramps at $100 \mathrm{~A} / \mathrm{s}$ on 5-cm aperture, 15-m long SSC dipole magnet prototypes DCA312 and DCA318. The pause current is $4800 \mathrm{~A}$ for magnet DCA312 and $5400 \mathrm{~A}$ for magnet DCA318.

TABLE III: Fitting parameters of the paused-ramp data of Fig. 5 by the function of Eq. (1).

\begin{tabular}{ccccccc}
\hline Magnet & $I_{0}$ & $I_{1}$ & $t_{1}$ & $I_{2}$ & $t_{2}$ & $\chi^{2}$ \\
Name & (A) & $(\mathrm{A})$ & $(\mathrm{s})$ & $(\mathrm{A})$ & $(\mathrm{s})$ & \\
\hline & & & & & & \\
DCA312 & 5032.1 & 1177.1 & 2.96 & 720.1 & 119.9 & 144 \\
DCA318 & 5664.0 & 78.1 & 4.14 & 481.1 & 215.8 & 0.88 \\
\hline
\end{tabular}


Figure 5 shows that, for both magnets, the quench current increases as a function of pause duration, but that the effect is much more accentuated for the type-A magnet (magnet DCA312) than for the type-B magnet (magnet DCA318). For magnet DCA312, a pause of 10 minutes results in a quench current increase of the order of $2000 \mathrm{~A}$, while, for magnet DCA318, the quench current barely increases by $1000 \mathrm{~A}$ after a 1-hour dwell. As we mentioned earlier, the main cause of this quench current recovery is thought to be cooling of the magnet coil during the pause. Since the two types of magnets are cooled the same way, a difference in behavior can be explained only if the heating during the ramp preceding the pause is different. Such interpretation thus implies that the ramp-induced heating is much more significant in the type-A magnet than in the type-B magnet.

Table III shows that, for both magnets, the time constants of the fitting functions are very similar, but that the amplitude of $I_{1}$ is much larger for magnet DCA312 than for magnet DCA318. The speculation here is that these time constants correspond to the cooling time constants of the system, $\tau_{1}$ being associated with the helium circulating in the coil cooling passage, between the coil and the beam tube, and $\tau_{2}$ being associated with the helium circulating in the yoke cooling passages, at the top and bottom of the iron yoke (see Figs. 1(a) and 1(b)). The fact that the time constants are nearly the same for magnets DCA312 and DCA318 confirm our assertion that these two magnets are cooled the same way, while the fact that the amplitude of $I_{1}$ is much larger for magnet DCA312 than for magnet DCA318 is consistent with the interpretation about the ramp-induced heating given above. 


\subsection{Staged ramps}

The second series of ramps, referred to as staged ramps are intended as control experiments for the so-called V-ramps described below. In a staged ramp, the magnet is first ramped to $I_{\mathrm{q}}\left(r_{0}\right)$ at a low ramp rate that does not cause the magnet to quench. The current is then held constant at $I_{\mathrm{q}}\left(r_{0}\right)$ for 10 minutes, after which the magnet is ramped to quench at $r_{0}$. The corresponding quench current is noted $I_{\mathrm{s}}\left(r_{0}\right)$.

Staged ramps were performed on both magnet DCA312 and magnet DCA318 for $r_{0}=$ $100 \mathrm{~A} / \mathrm{s}$. In the case of magnet DCA312, the initial ramp was to $5013 \mathrm{~A}$ at $4 \mathrm{~A} / \mathrm{s}$, and the final quench current was $7054 \mathrm{~A}$. For magnet DCA318, the initial ramp was to $5600 \mathrm{~A}$ at $16 \mathrm{~A} / \mathrm{s}$, and the final quench current was $6290 \mathrm{~A}$.

\subsection{V-ramps}

The third series of ramps, referred to as V-ramps, are intended to be compared to the stage ramps in order to determine if the dominant mechanism is eddy current heating. In a $V$-ramp, the magnet is first ramped to $I_{\mathrm{s}}\left(r_{0}\right)$ at a low ramp rate that does not cause the magnet to quench. The current is then held constant at $I_{\mathrm{s}}\left(r_{0}\right)$ for 10 minutes. After the pause, the magnet is ramped down from $I_{\mathrm{s}}\left(r_{0}\right)$ to $I_{\mathrm{q}}\left(r_{0}\right)$ at $r_{0}$, and ramped up again from $I_{\mathrm{q}}\left(r_{0}\right)$ to quench at $r_{0}$. The corresponding quench current is noted $I_{\mathrm{v}}\left(r_{0}\right)$.

The main difference between staged ramps and $V$-ramps is that, in the case of staged ramps, the ramp from $I_{\mathrm{q}}\left(r_{0}\right)$ to quench takes place just after a pause, while, in the case of $V$-ramps, it is preceded by a down ramp at $r_{0}$. If the dominant mechanism is eddy-current heating, the eddy currents generated during the down ramp are expected to pre-heat the magnet coil, reducing the temperature margin and the quench current. On the contrary, if $I_{\mathrm{v}}$ 
turns out to be larger than $I_{\mathrm{s}}$, it is a strong indication that the mechanism is not dominated by eddy current heating.

As for staged-ramps, $V$-ramps were performed on both magnet DCA312 and magnet DCA318 for $r_{0}=100 \mathrm{~A} / \mathrm{s}$. In the case of magnet DCA312, the initial ramp was at $4 \mathrm{~A} / \mathrm{s}$ up to $7059 \mathrm{~A}$. After the pause, the magnet was ramped down to $5013 \mathrm{~A}$, and the final quench current was $6840 \mathrm{~A}$, thus about $200 \mathrm{~A}$ below $I_{\mathrm{s}}(100 \mathrm{~A} / \mathrm{s})$. In the case of magnet DCA318, the initial ramp was at $16 \mathrm{~A} / \mathrm{s}$ up to $6250 \mathrm{~A}$. After the pause, the magnet was ramped down to $5600 \mathrm{~A}$, and the final quench current was $6700 \mathrm{~A}$, thus more than $400 \mathrm{~A}$ above $I_{\mathrm{S}}(100 \mathrm{~A} / \mathrm{s})$.

The above results show that, for the type-A magnet (magnet DCA312), the introduction of the down-ramp caused a reduction in quench current, while, for the type-B magnet (magnet DCA318), it caused an increase. The behavior of the type-A magnet is thus consistent with what can be expected from eddy current heating, while that of the type-B magnet shows that it cannot be the dominant mechanism. This interpretation of the $V$-ramp data corroborates that of the paused-ramp data, from which it was concluded that the ramp-induced heating played a more significant role in the type-A magnet than in the type-B magnet.

\subsection{Pre-cycled ramps}

The fourth series of ramps, referred to as pre-cycled ramps, are intended to study the effect of a pre-cycle on the quench current. Let $I_{\mathrm{f}}$ designate a current value below the plateau quench current at $4.35 \mathrm{~K}$. In a pre-cycled ramp, the magnet is first ramped to $I_{\mathrm{f}}$ at a rate (or a succession of rates) which does not (do not) cause the magnet to quench, without being 
too time consuming. The current is then held constant at $I_{\mathrm{f}}$ for a duration, $\tau_{\mathrm{f}}$, before being ramped down to $25 \mathrm{~A}$ at $r_{0}$. After another pause of 10 minutes at $25 \mathrm{~A}$, the magnet is ramped up to quench at $r_{0}$.

Pre-cycled ramps were performed on magnet DCA317, with $r_{0}=250 \mathrm{~A} / \mathrm{s}$, and on magnet DCA318, with $r_{0}=100 \mathrm{~A} / \mathrm{s}$. In the case of magnet DCA317, the ramp rate of the pre-cycles' up-ramps was $16 \mathrm{~A} / \mathrm{s}$, while, for magnet $\mathrm{DCA} 318$, it was $16 \mathrm{~A} / \mathrm{s}$ up to $6000 \mathrm{~A}$, and $1 \mathrm{~A} / \mathrm{s}$ above $6000 \mathrm{~A}$. Note, however, that it was found after the tests, that the ramp rate of the pre-cycles' down ramps of magnet DCA317 was only $110 \mathrm{~A} / \mathrm{s}$ instead of the intended $250 \mathrm{~A} / \mathrm{s}$.

Figure 6(a) presents a summary plot of quench current versus pre-cycle flattop current for a pre-cycle flattop duration of 10 minutes. It appears that, for both magnets, the quench current increases as a function of pre-cycle flattop current but that the effect is much more accentuated for the type-B magnet (magnet DCA318) than for the type-A magnet (magnet DCA317). In the case of magnet DCA318, the quench current increases by about $1400 \mathrm{~A}$ between a ramp with no pre-cycle and a ramp with a $7300 \mathrm{~A}$ pre-cycle, while, in the case of magnet DCA312, the increase is only of the order of $300 \mathrm{~A}$ between a ramp with no pre-cycle and a ramp with a $7000 \mathrm{~A}$ pre-cycle. (As a comparison, the plateau quench current of magnet DCA318 at $4.35 \mathrm{~K}$ is $7450 \mathrm{~A}$, while that of magnet DCA317 is 7375 A.)

Figure 6(b) presents a summary plot of quench current versus pre-cycle flattop duration for selected pre-cycle flattop currents (7000 A for magnet DCA317; $6000 \mathrm{~A}$ and 
$7300 \mathrm{~A}$ for magnet DCA318). In all cases, the quench current appears to increase as a function of pre-cycle flattop duration.

In the case of magnet DCA317, a series of ramps with identical pre-cycles $\left(I_{\mathrm{f}}=\right.$ $7000 \mathrm{~A}$ and $\tau_{\mathrm{f}}=600 \mathrm{~s}$ ), but varying $25-\mathrm{A}$ pause duration were also performed. The results are displayed in Fig. 6(c). Here, it appears that the quench current decreases as a function of 25-A pause duration.

Finally, magnet DCA318 was subjected to a ramp with a pre-cycle to $7300 \mathrm{~A}$ for $600 \mathrm{~s}$ at the standard ramp rates, followed by a $600-\mathrm{s}$ pause at $25 \mathrm{~A}$; and a ramp to quench at $16 \mathrm{~A} / \mathrm{s}$. The resulting quench current $(7447 \mathrm{~A})$ and quench start localization (inner coil pole turn) are similar to that of the plateau quenches. This last ramp thus shows that the introduction of a 7300-A pre-cycle prior to a ramp, which normally would cause a degraded quench, greatly improves the performance and leads to a plateau-like quench.

The above results provide further evidences that the type- $\mathrm{B}$ behavior is not dominated by ramp-induced heating. They also show that the quench currents of magnet DCA318 are strongly affected by the excitation history and by how high in current the magnet has been cycled prior to the ramp to quench. Magnet DCA317 also exhibits similar symptoms, but they are very attenuated, and they do not rule out eddy current heating as the dominant mechanism of type-A behavior. 
(a)

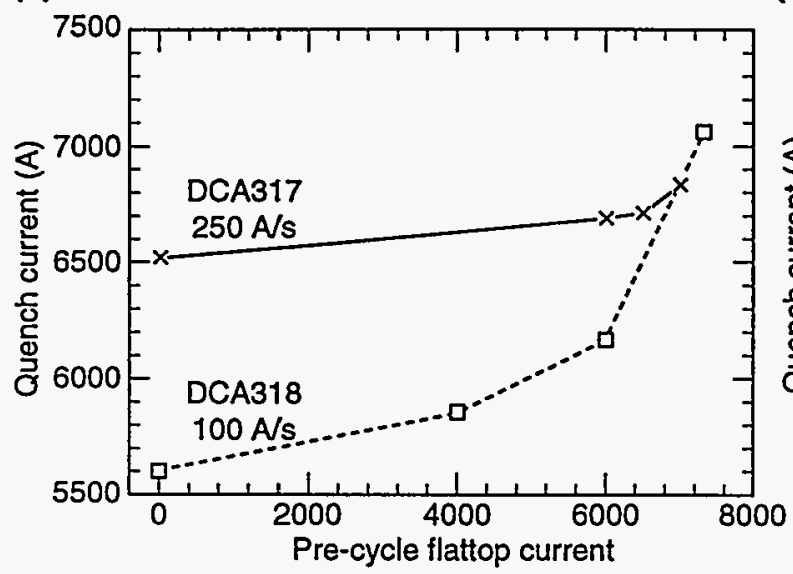

(c)

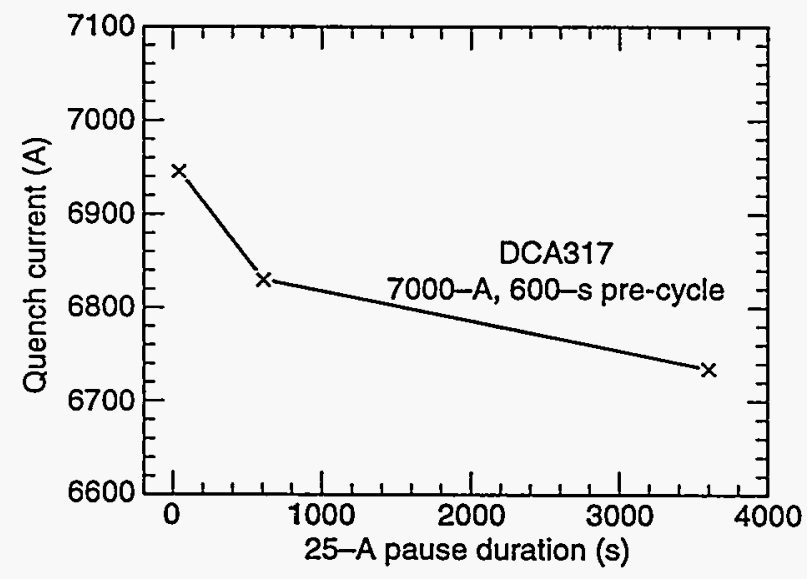

(b)

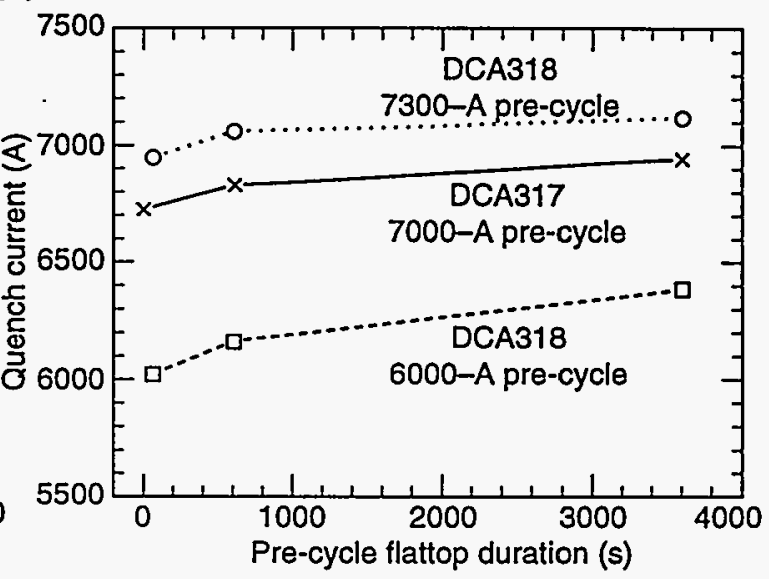

FIGURE 6: Quench current versus pre-cycle parameters for a series of pre-cycled ramps on 5-cm aperture, 15-m long SSC dipole magnet prototypes DCA317 and DCA318: a) influence of pre-cycle flattop current, b) influence of pre-cycle flattop duration, and c) influence of 25-A pause duration. If not otherwise specified, the pre-cycle flattop current is $7000 \mathrm{~A}$ for magnet DCA317 and $7300 \mathrm{~A}$ for magnet DCA318, and the durations of the pre-cycle flattop and 25-A pause are $600 \mathrm{~s}$. The rate of the final ramp to quench is $250 \mathrm{~A} / \mathrm{s}$ for magnet DCA317 and $100 \mathrm{~A} / \mathrm{s}$ for magnet DCA318. 


\subsection{Definitions}

In the long, almost straight, section of the magnet, the field, $\mathbf{B}$, can be considered as twodimensional, and is conveniently represented by a multipole expansion

$$
\mathbf{B}(x+\mathrm{i} y)=B_{\mathrm{y}}(x, y)+\mathrm{i} B_{\mathrm{x}}(x, y)=\sum_{n=0}^{+\infty}\left(B_{\mathrm{n}}+\mathrm{i} A_{\mathrm{n}}\right)\left(\frac{x+\mathrm{i} y}{R_{\mathrm{ref}}}\right)^{\mathrm{n}},
$$

where $B_{\mathrm{x}}$ and $B_{\mathrm{y}}$ are the $\mathrm{x}$ - and $\mathrm{y}$-components of the field, $B_{\mathrm{n}}$ and $A_{\mathrm{n}}$ are the normal and skew $2(\mathrm{n}+1)$-pole fields, and $R_{\text {ref }}$ is the reference radius. (For the SSC magnets, $R_{\text {ref }}=$ $1 \mathrm{~cm}$.) The rectangular coordinate system $(0, x, y, z)$ is defined so that the $z$-axis is parallel to the ideal beam orbit and $O$ is at the magnet center.

The symmetries of a cosine-theta distribution of conductors are such that only even normal multipole fields, also called allowed multipole fields, can be non-zero. In real magnets, however, manufacturing errors and non-uniformities in the material properties can result in violations of these symmetries and lead to non-zero un-allowed multipole fields. Typical examples of such violations are: a top/bottom asymmetry, which results in a nonzero skew quadrupole field $\left(A_{1}\right)$, and a left/right asymmetry, which results in a non-zero normal quadrupole field $\left(B_{1}\right)$.

For SSC dipole magnets, the dipole field is expected to be about $10^{4}$ times larger than any other multipole fields. Hence, it is customary to introduce the dimensionless multipole coefficients, $a_{\mathrm{n}}$ and $b_{\mathrm{n}}$, defined as

$$
a_{\mathrm{n}}=10^{4} \frac{A_{\mathrm{n}}}{B_{0}},
$$


and

$$
b_{\mathrm{n}}=10^{4} \frac{B_{\mathrm{n}}}{B_{0}}
$$

\subsection{Magnetic measurements}

The magnetic measurements reported here were performed using a rotating coil system developed by BNL. 27 This system, called the mole, consists of a tangential winding and two dipole-bucking windings. The tangential winding of the $F$-series moles (used for most of the measurements) has 30 turns and a $15^{\circ}$ opening angle; it is sensitive to all multipole fields of interest. The diametrical dipole-bucking windings have 3 turns; they are mainly sensitive to dipole fields and their allowed harmonics. The three windings have about the same mean radius, of the order of $1.2 \mathrm{~cm}$, and about the same mean length, of the order of $1 \mathrm{~m}$; variations are measured and corrected in software. The coil array rotates with a 3.5-s period. The voltages induced in the three windings, together with the magnet current, are measured simultaneously by means of digital multimeters. The multimeters have a 7.5- or 8.5-digit resolution and are configured to integrate the input readings over 1 power line cycle. The data acquisition is triggered by an incremental optical angle encoder with a 12-arc-second resolution. There are 128 equally spaced acquisitions per rotation. Some of the FNAL measurements were taken using an older version of the mole, referred to as $B$-series. The windings of the $B$-series mole have a length of about $0.6 \mathrm{~m}$; the tangential winding has 40 turns and the dipole-bucking windings have 4 turns.

Multipole fields of order up to 32 are estimated by taking Fast Fourier Transforms (FFT) ${ }^{28}$ of the voltage data from every other rotation. To determine the dipole fields, the data from one of the dipole-bucking windings are sufficient. Higher-order multipole fields 
are determined from a linear combination of the three voltages, such that the dipole terms of the combined FFTs are forced to be zero. This technique, referred to as digital bucking, has proven to be successful in achieving the required accuracy for multipole coefficients above the dipole. ${ }^{29}$ Bucking is needed for a number of reasons but most importantly to reduce sensitivity to mechanical vibrations and power supply ripple.

In preparation for the measurements, the magnet is mounted on the test stand so that the dipole field is approximately vertical. Also, the mole, which is equipped with two high resolution gravity sensors, is positioned within the magnet bore so that the housing of the angle encoder is aligned with respect to gravity. After processing the data of a given rotation, it is customary to perform a number of transformations to report the multipole fields in a standard coordinate system. The first is a translation to a system where either the 16-pole fields $\left(A_{7}\right.$ and $\left.B_{7}\right)$ or the 20-pole fields $\left(A_{9}\right.$ and $\left.B_{9}\right)$ are forced to be zero. This centers the mole data in the magnet aperture. It is followed by a rotation to a system where the skew dipole field $\left(A_{0}\right)$ is forced to be zero. This aligns the $y$-axis with the dipole field. Finally, the directions of the axes are changed as required so that the $x$-axis points towards the right-hand side, and the $y$-axis points upward when facing the magnet from the non-lead end.

The test plan calls for a number of magnetic measurements as a function of current and ramp rate. It includes measurements following a test sequence representative of a Collider operating cycle. This sequence starts with a cleansing quench to erase any previous superconductor magnetization (see below). The magnet is then pre-cycled to a current of $6500 \mathrm{~A}$, for a duration of 5 minutes, simulating a colliding beam cycle. Next, it is 
ramped down to $115 \mathrm{~A}$ for a 2 -minute dwell, ramped up to $620 \mathrm{~A}$ for a 10-minute preinjection porch, and ramped up again to $635 \mathrm{~A}$ for a 1-hour injection porch, simulating a beam injection cycle from the HEB to the Collider. At the end of the injection porch, the current is ramped up again to $6500 \mathrm{~A}$, and then ramped down to $115 \mathrm{~A}$, to simulate the next colliding beam cycle. The ramp rate is $4 \mathrm{~A} / \mathrm{s}$ except for the ramp from 620 to $635 \mathrm{~A}$ that is performed at $1 \mathrm{~A} / \mathrm{s}$. The test plan also includes a test sequence aimed at measuring the ramp-rate sensitivity of the multipole fields. This test sequence starts by a cleansing quench and a pre-cycle identical to that of the previous test sequence. However, the 115-A dwell is now followed by a series of sawtooth ramps between $115 \mathrm{~A}$ and $6500 \mathrm{~A}$ executed at increasing ramp rates (typically: $4 \mathrm{~A} / \mathrm{s}, 8 \mathrm{~A} / \mathrm{s}, 16 \mathrm{~A} / \mathrm{s}, 32 \mathrm{~A} / \mathrm{s}$ and $64 \mathrm{~A} / \mathrm{s}$ ).

\subsection{Expected current dependence of magnetic field}

The main contribution to the field of superconducting particle accelerator magnets comes from the transport current circulating in the coils. The transport-current field is expected to increase linearly as a function of current (see section 7.2). Its multipole coefficients, referred to as geometric multipole coefficients, are expected to assume constant values. In real magnets, however, a number of current-dependent effects are known to take place that distort the transport-current field and result in current-dependent multipole coefficients. Among them are the effects of persistent magnetization currents and of iron yoke saturation.

According to the critical-state model, ${ }^{30}$ persistent magnetization currents are induced at the periphery of the superconducting filaments each time the field to which the filaments are exposed is varied. These magnetization currents distribute themselves, with a density 
equal to the critical current density of the superconductor, in order to screen the filaments' cores from the local field change. The filaments, with their shells of persistent magnetization currents, then behave as magnetic doublets which contribute to-and distort-the central field. A model, originally developed at Deutsches ElektronenSynchrotron Laboratory (DESY), ${ }^{31-33}$ allows the orientation and strength of these magnetic doublets, and their contributions to the central field, to be determined. The computation relies on the critical state model but the persistent-magnetization-current shells are assumed to be elliptical. The distribution of magnetic doublets follows the symmetries of the transport-current field and, if the properties of the superconductor are uniform, only the allowed multipole fields are affected. Also, since the doublets' strength is proportional to the critical current density, the effect decreases as the transport current, and, thus, the transport-current field, increase. The DESY model was very successful in predicting the current dependence of the multipole coefficients of the superconducting magnets for the Hadron Electron Ring Accelerator (HERA). It is also quite successful in predicting the behavior of most of the SSC dipole and quadrupole magnet prototypes. ${ }^{14}$

Above $4000 \mathrm{~A}$, the transport-current field produced by the coil is large enough to saturate the iron yoke. Since the field is the largest at the pole, iron saturation is first felt there. This results in a positive contribution to the normal sextupole coefficient $\left(b_{2}\right)$. The return flux through the midplane causes it to saturate as well. At currents of the order of $6500 \mathrm{~A}$, midplane saturation overcomes pole saturation resulting in a net negative $b_{2} \cdot{ }^{34} \mathrm{The}$ present magnetic design, however, includes cut-outs at the midplane of the iron yoke to force the midplane saturation to occur sooner, and thus, to compensate partially the effect 
on $b_{2}$ of the pole saturation. ${ }^{16}$ The iron saturation is known also to affect the normal decapole coefficient $\left(b_{4}\right)$ but to a lesser extent. The observed $b_{2}$ and $b_{4}$ saturations conform to predictions for both the BNL and the FNAL design.

Another predicted effect is that, at high current, flux lines start to leak out of the cold mass. As the cold mass is not centered within the cryostat, and the cryostat is made of low carbon steel, the flux lines become slightly distorted. This distortion, which violates the top/bottom symmetry, results in a decrease of the skew quadrupole coefficient $\left(a_{1}\right)$. The observed $a_{1}$ saturation of the FNAL magnets is within the prediction range. For the BNL magnets, however, the $a_{1}$ saturation appears to vary magnet to magnet, as well as within the magnets' lengths. These variations can be shown to be correlated with local top/bottom asymmetries in the weight of the laminated iron yoke modules, which, in the BNL design, are horizontally split. ${ }^{35}$ Similar distortions are seen at high current in the normal quadrupole coefficient $\left(b_{1}\right)$ of the FNAL magnets. They are attributed to left/right asymmetries in the packing factor of the laminated iron yoke modules, which, in the FNAL design, are vertically split.

\subsection{Observed ramp-rate dependence of magnetic field}

Figures 7(a) through 7(f) present summary plots of quadrupole, sextupole, and decapole coefficients versus current for a series of sawtooth ramps executed at increasing ramp rates on magnet DCA318. Figures 8(a) through 8(f) present similar plots for magnet DCA312. 
(a)

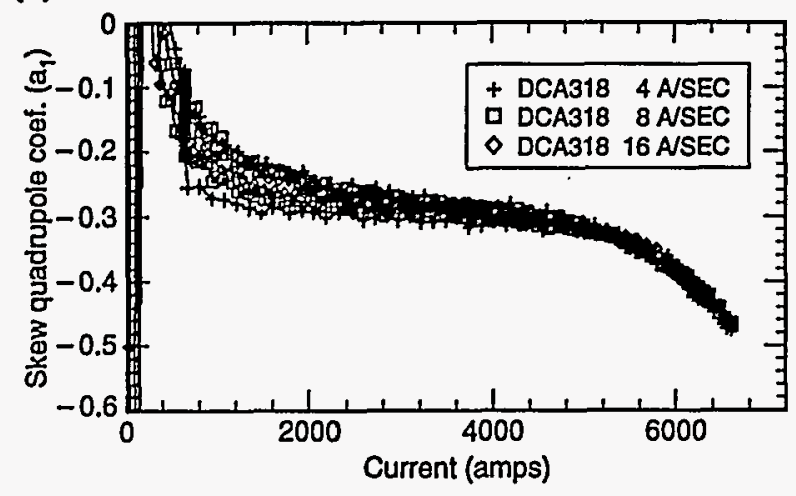

(c)

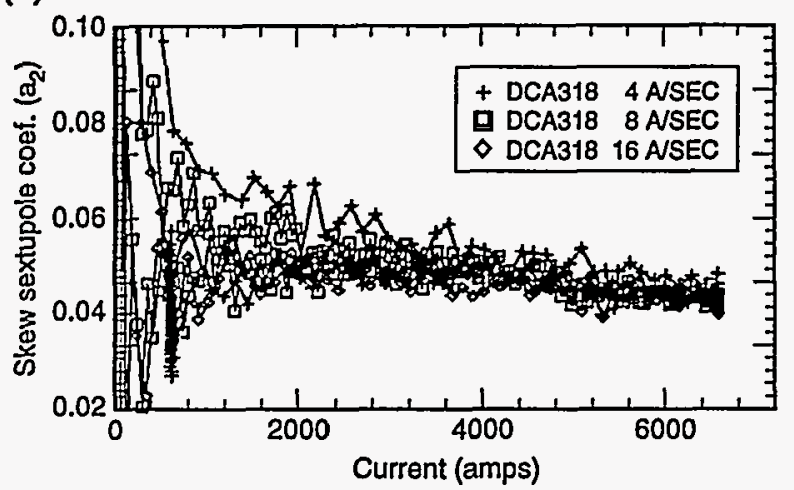

(e)

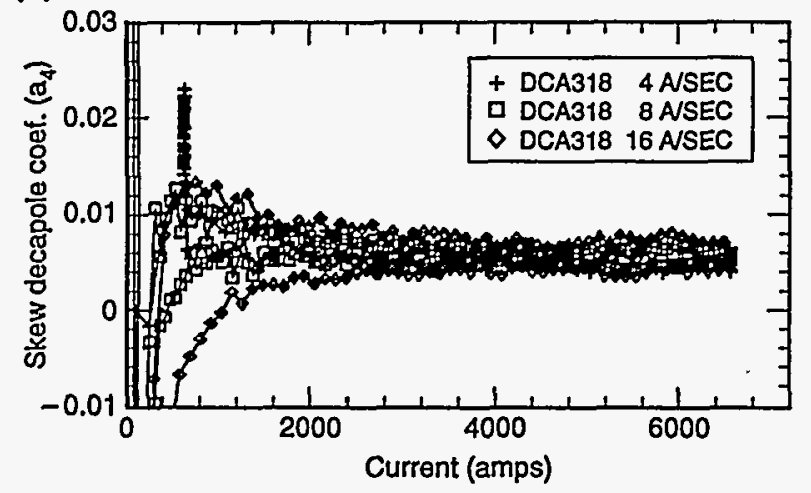

(b)

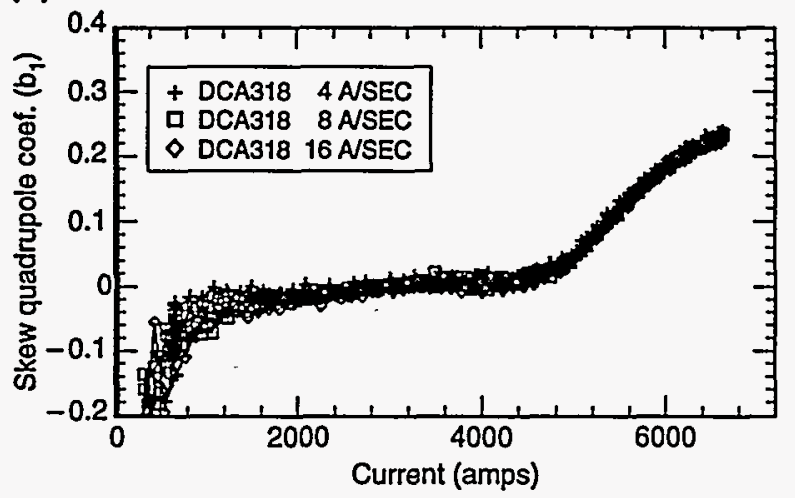

(d)

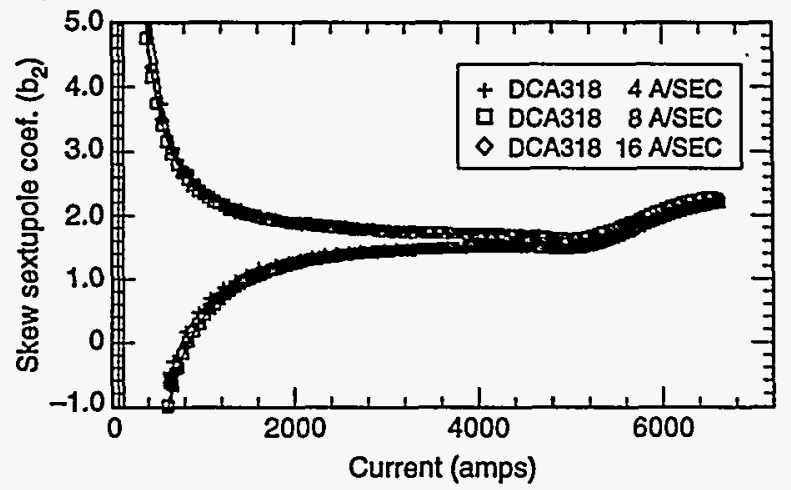

(f)

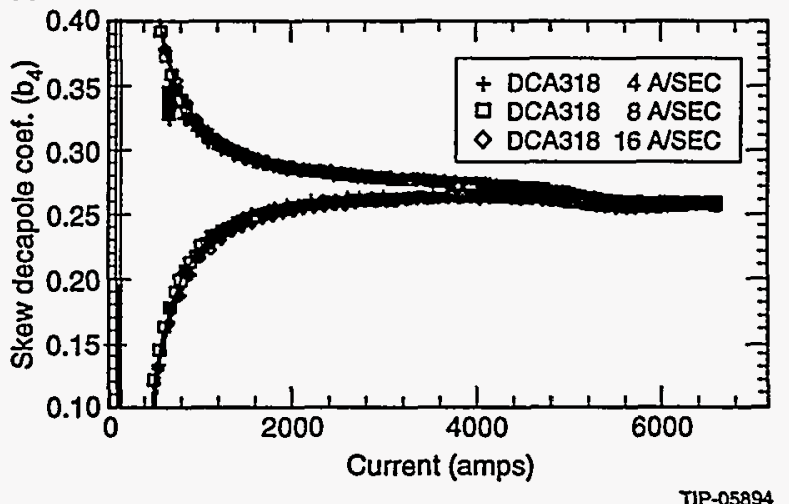

FIGURE 7: Multipole coefficients as a function of current as measured on magnet DCA318 during a series of sawtooth ramps at increasing ramp rates: a) skew quadrupole coefficient $\left(a_{1}\right)$, b) normal quadrupole coefficient $\left(b_{1}\right)$, c) skew sextupole coefficient $\left(a_{2}\right)$, d) normal sextupole coefficient $\left(b_{2}\right)$, e) skew decapole coefficient $\left(a_{4}\right)$, and f) normal decapole coefficient $\left(b_{4}\right)$. The multipole coefficients are in dimensionless units (see Eqs. (3)). 
(a)

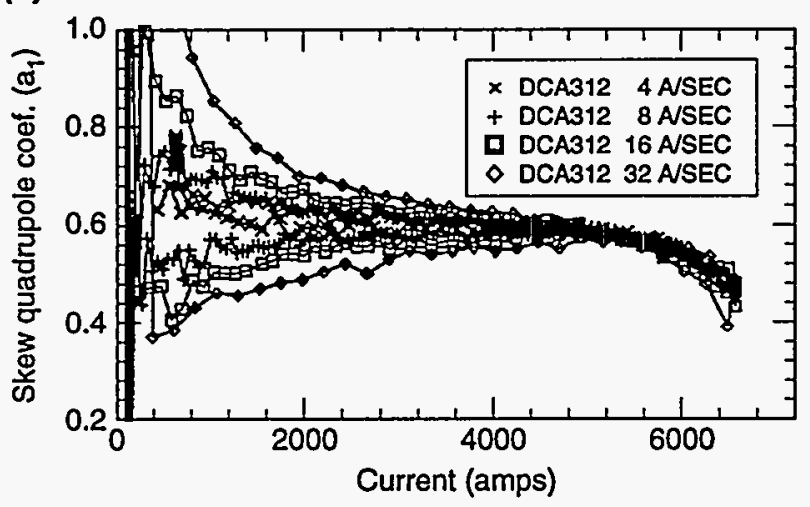

(c)

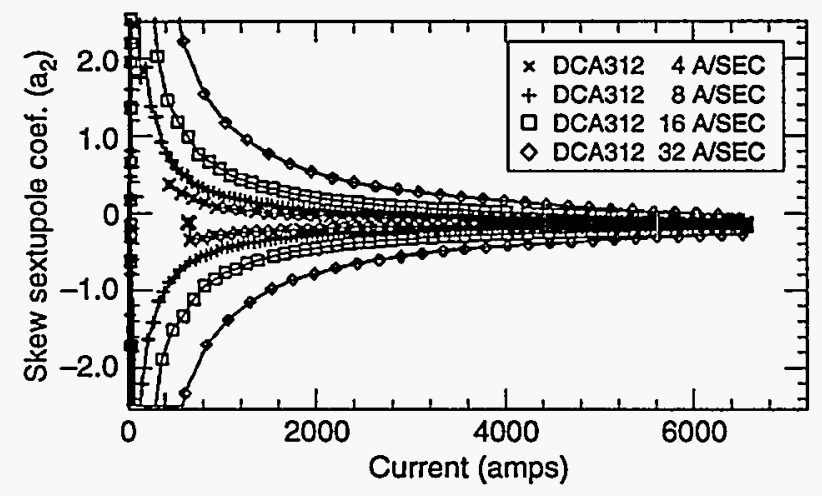

(e)

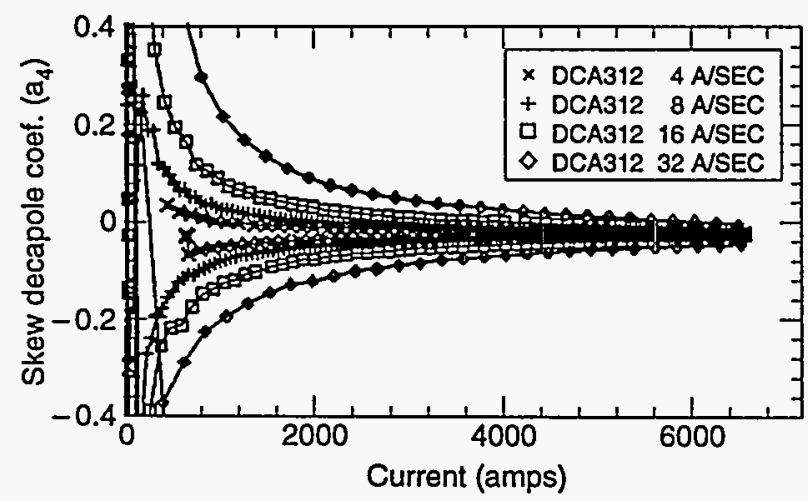

(b)

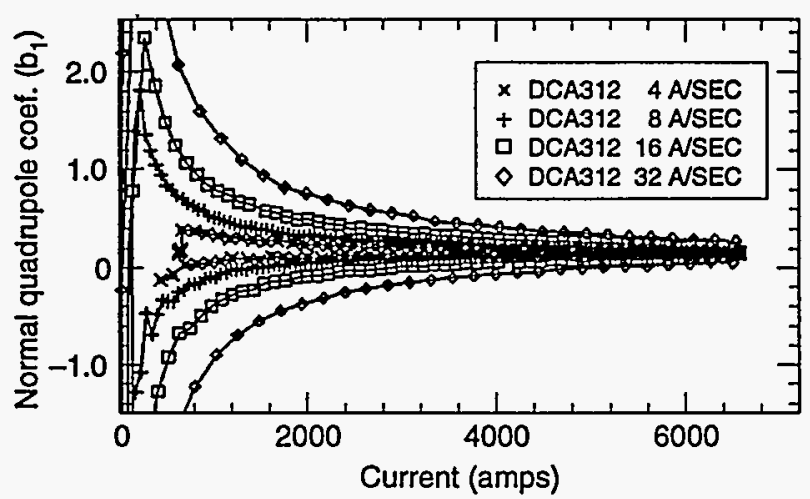

(d)

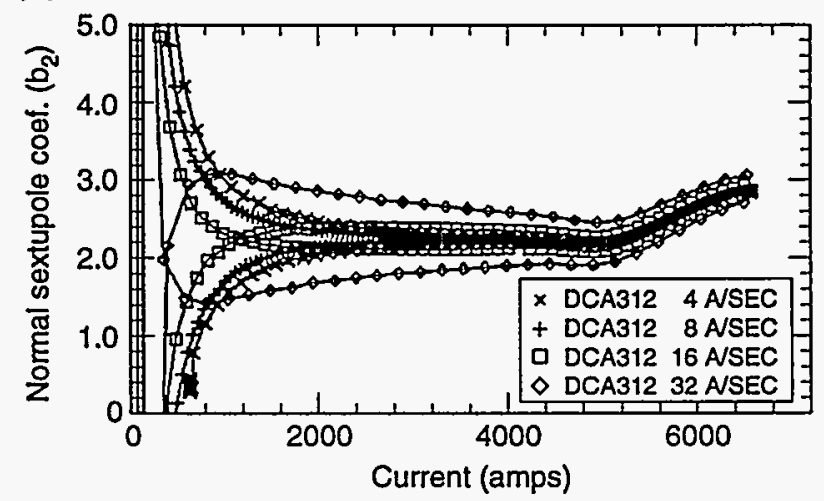

(f)

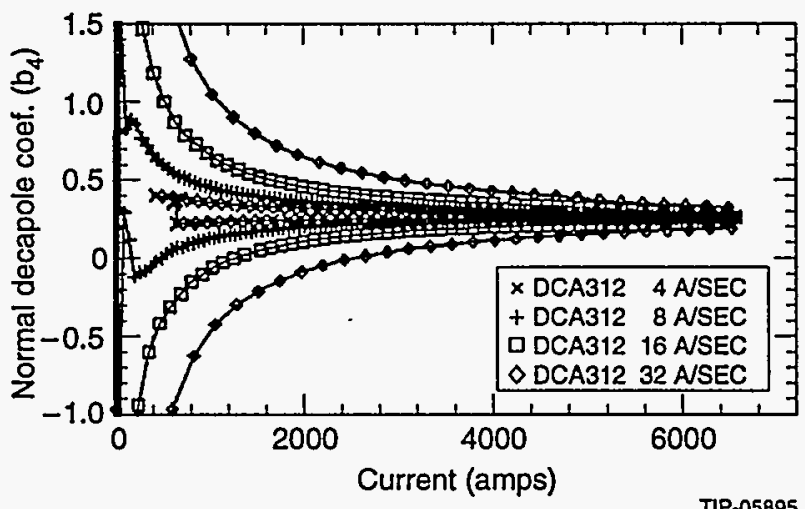

FIGURE 8: Multipole coefficient as a function of current as measured on magnet DCA312 during a series of sawtooth ramps at increasing ramp rates: a) skew quadrupole coefficient $\left(a_{1}\right)$, b) normal quadrupole coefficient $\left(b_{1}\right)$, c) skew sextupole coefficient $\left(a_{2}\right)$, d) normal sextupole coefficient $\left(b_{2}\right)$, e) skew decapole coefficient $\left(a_{4}\right)$, and f) normal decapole coefficient $\left(b_{4}\right)$. The multipole coefficients are in dimensionless units (see Eqs. (3)). 
Looking first at Fig. 7, it appears that the multipole coefficients of magnet DCA318 show little sensitivity to the ramp rate. It appears also that the allowed sextupole and decapole coefficients ( $b_{2}$ and $\left.b_{4}\right)$ exhibit an hysteretic behavior as a function of current, while none of the un-allowed multipole coefficients do. This behavior is consistent with what is expected from the effects of persistent magnetization currents, and the amplitudes of the $b_{2}$ and $b_{4}$ hystereses are in good agreement with the predictions. ${ }^{14}$ In addition, most of the multipole coefficients exhibit distortions for currents above $4000 \mathrm{~A}$. These distortions are consistent with what is expected from the iron yoke saturation effects discussed above.

Looking now at Fig. 8 , it appears that, along with $b_{2}$ and $b_{4}$, most of the un-allowed multipole coefficients of magnet DCA312 exhibit an hysteretic behavior as a function of current. It appears also that, in the current range where the iron saturation effects are negligible, the hystereses are symmetrical about an axis parallel to the current-axis and that their widths strongly depend on the ramp rate. In addition, it appears that, at 16 and $32 \mathrm{~A} / \mathrm{s}$ in the case of $b_{2}$, and for all the measured ramp rates in the case of $b_{4}$, the hystereses are described in a direction opposite to what is expected from the effects of persistent magnetization currents.

The behavior of magnet DCA318 is typical of the type-B magnets. Magnets DCA313 through DCA315 exhibit behaviors similar to that of magnet DCA312, with anomalous hystereses of most of the multipole coefficients, even at $4 \mathrm{~A} / \mathrm{s}$. These magnets were the type-A magnets with the most serious quench current degradation at high ramp rates. Magnet DCA207 was only measured at $4 \mathrm{~A} / \mathrm{s}$, and does not exhibit anomalous hystereses. 
Magnet DCA317, which was the type-A magnet with the mildest quench current degradation, has a dynamic field behavior resembling that of magnet DCA318.

\subsection{Investigation of type-A behavior}

Let us now investigate in more detail the dynamic field behavior of the type-A magnets. Figures 9(a) and 9 (b) present plots of skew quadrupole and normal decapole coefficients for currents in the range [300 A, $900 \mathrm{~A}]$ as measured on type-A magnets DCA315 (Fig. 9(a)) and DCA312 (Fig. 9(b)) during a test sequence representative of the Collider operating cycle. The data reported here correspond to measurements taken after the 115-A dwell following the pre-cycle. The multipole coefficients have been shifted along the $y$-axis using

$$
a_{\mathrm{n}, \mathrm{s}}=a_{\mathrm{n}}-\frac{a_{\mathrm{n}, \mathrm{up}}+a_{\mathrm{n}, \mathrm{dwn}}}{2},
$$

and

$$
b_{\mathrm{n}, \mathrm{s}}=b_{\mathrm{n}}-\frac{b_{\mathrm{n}, \mathrm{up}}+b_{\mathrm{n}, \mathrm{dwn}}}{2} \text {, }
$$

where $a_{\mathrm{n}, \mathrm{up}}$ and $b_{\mathrm{n} \text {,up }}$ designate the average values of $a_{\mathrm{n}}$ and $b_{\mathrm{n}}$ during the up-ramp from $2 \mathrm{kA}$ to $3 \mathrm{kA}$ and $a_{\mathrm{n}, \mathrm{dwn}}$ and $b_{\mathrm{n}, \mathrm{dwn}}$ designate the average values of $a_{\mathrm{n}}$ and $b_{\mathrm{n}}$ during the down-ramp from $3 \mathrm{kA}$ to $2 \mathrm{kA}$. This shift is applied to center the hystereses about the current-axis and can be interpreted as a subtraction of the geometric components of the multipole coefficients. For comparison, the dashed lines correspond to the predicted effects of the persistent magnetization currents. 
(a)

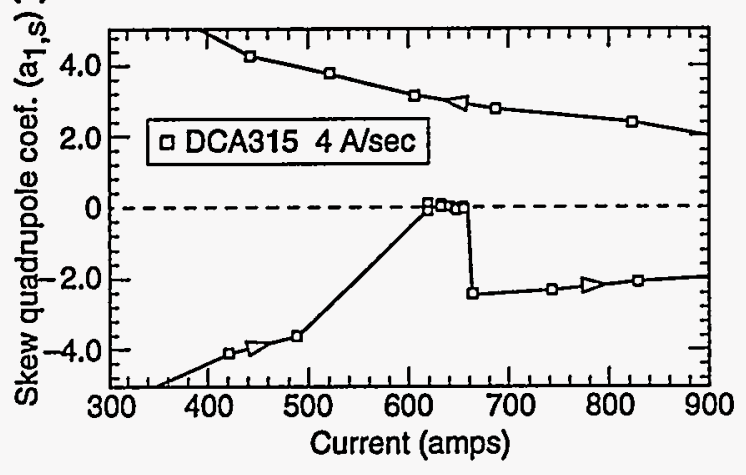

(b)

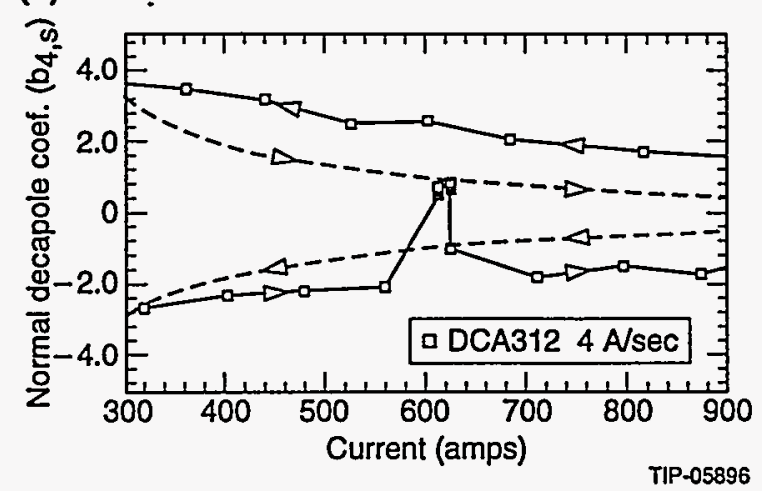

FIGURE 9: Multipole coefficients as a function of current as measured during a test sequence representative of the Collider operating cycle: a) skew quadrupole coefficient (magnet DCA315), and b) normal decapole coefficient (magnet DCA312). The multipole coefficients are in dimensionless units (see Eqs. (3)). The plots are shifted along the $y$-axis to remove the geometric component. The dashed lines correspond to the predicted effects of the persistent magnetization currents.

Looking first at Fig. 9(a) and following the current ramp at $4 \mathrm{~A} / \mathrm{s}$ between $300 \mathrm{~A}$ and $620 \mathrm{~A}$, it is seen that the skew quadrupole coefficient describes the lower branch of an hysteresis curve. Upon reaching the pre-injection porch, the ramp is stopped, and $a_{1, \mathrm{~s}}$ appears to go to zero. It stays at zero for the duration of the pre-injection and injection porches, during which the current is held constant. For the particular set of data presented here, at the end of the injection porch, the current was first increased by steps of $1 \mathrm{~A}$ from 635 to $655 \mathrm{~A}$, and magnetic measurements were taken on each of the steps. Throughout the step-by-step increase, $a_{1, \mathrm{~s}}$ appears to stay at zero. Upon reaching $655 \mathrm{~A}$, the $4-\mathrm{A} / \mathrm{s}$ ramp was resumed and $a_{1, \mathrm{~s}}$ appears to leap back to the anomalous hysteresis, which it keeps describing until the end of the cycle. In summary, the anomalous behavior of the skew 
quadrupole coefficient is only observed while the current is ramped and it ceases when the current ramp is stopped.

Similar observations can be made when looking at Fig. 9(b). As mentioned above, the normal decapole coefficient of magnet DCA312 appears to describe, while ramping at $4 \mathrm{~A} / \mathrm{s}$, a hysteresis of direction opposite to what is expected from the effects of persistent magnetization currents. The data of Fig. 9(b) show that, upon reaching the pre-injection porch, $b_{4, \mathrm{~s}}$ leaps toward the predicted curve and stays in its neighborhood for the duration of both the pre-injection and injection porches. They show also that, upon resumption of the 4-A/s ramp, $b_{4, \mathrm{~s}}$ leaps back to the anomalous hysteresis. As for the skew quadrupole coefficient, the anomalous behavior of the normal decapole coefficient is only observed while the current is ramped and it ceases when the current ramp is stopped.

The above observations provide further evidences that the anomalous field behavior of the type-A magnet is ramp-rate related. They also show that the time constant of the phenomenon here involved is less than $20 \mathrm{~s}$, which corresponds to the time between two successive data points on the plots.

Let us now go back to the multipole fields by re-scaling the data of Fig. 8 using

$$
A_{\mathrm{n}, \mathrm{I}}=B_{0} a_{\mathrm{n}, \mathrm{s}},
$$

and

$$
B_{\mathrm{n}, \mathrm{r}}=B_{0} b_{\mathrm{n}, \mathrm{s}}
$$

where $A_{\mathrm{n}, \mathrm{r}}$ and $B_{\mathrm{n}, \mathrm{r}}$ are expressed in Gauss. Re-scaled plots of the skew and normal sextupole coefficients of magnet DCA312 are presented in Figs. 10(a) and 10(b). It appears 
that, for the un-allowed $A_{2, \mathrm{r}}$, where no effects are expected from the persistent magnetization currents, the width of the anomalous hysteresis is roughly constant as a function of current, and increases quasi-linearly as a function of ramp rate. The situation is more complicated for the allowed $B_{2, \mathrm{r}}$, where there is a large contribution from the persistent magnetization currents, which is expected to decrease as a function of current. However, there too, it can be verified that the amplitude of the ramp-rate related effect is roughly constant as a function of current and that it increases quasi-linearly as a function of ramp rate. Similar observations can be made for all the other re-scaled multipole fields.

(a)

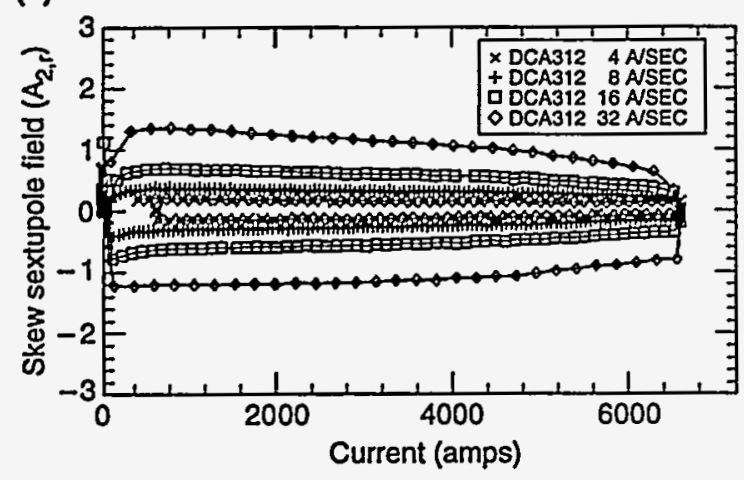

(b)

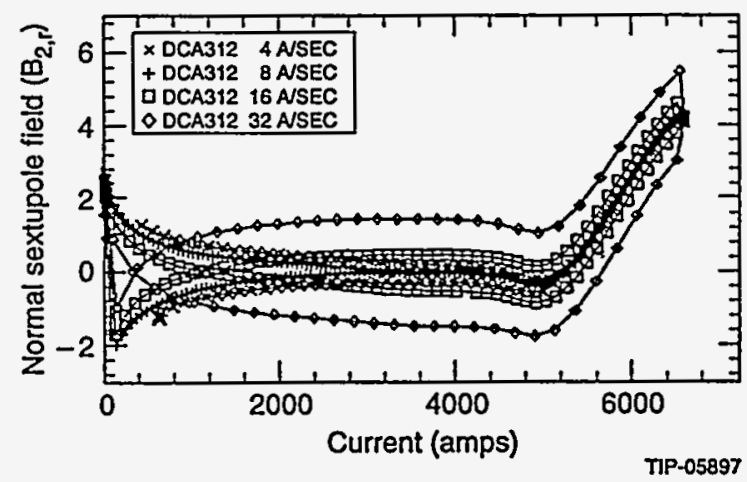

FIGURE 10: Re-scaled multipole fields as a function of current from measurements during a series of sawtooth ramps at increasing ramp rates performed on magnet DCA312: a) skew sextupole field $\left(A_{2, \mathrm{r}}\right)$, and $\left.\mathrm{b}\right)$ normal sextupole field $\left(B_{2, \mathrm{r}}\right)$. The multipole fields are in Gauss. The re-scaling corresponds to a subtraction of the transport-current contribution.

In addition, in the case of magnet DCA312, a set of magnetic measurements as a function of ramp rate was taken at three axial positions along the magnet: 1) $5.5 \mathrm{~m}$ from the magnet center toward the lead end, 2) $0.5 \mathrm{~m}$ from the magnet center toward the non-lead end, and 3) $5.5 \mathrm{~m}$ from the magnet center toward the non-lead end. (The distances are quoted with respect to the center of the measuring coil array). At the three positions, 
behaviors similar to that depicted above were observed. Figures 11(a) and 11(b) present summary plots as a function of ramp rate of the widths of the re-scaled skew and normal sextupole hystereses, $\Delta A_{2, \mathrm{r}}$ and $\Delta B_{2, \mathrm{r}}$, for the three sets of measurements. The hysteresis width is calculated as the average difference between the up- and down-ramp re-scaled multipole fields for currents in the range [2 kA, $3 \mathrm{kA}$ ]. It appears that, although the sign and amplitude of the effect vary from one position to the other, the ramp-rate dependence is always quasi-linear. It appears also that when fitting the data with first order polynomials, the intercepts are the same for the three positions: zero for the skew sextupole field and a negative number for the normal sextupole field. This is consistent with what can be expected from the effects of persistent magnetization currents. Similar observations can be made on the other multipole fields that can be characterized by the fitted slopes of $\Delta A_{n, I}$ and $\Delta B_{\mathrm{n}, \mathrm{r}}$ as a function of ramp rate. Note, however, that since the hysteresis width includes the contributions from both up- and down-ramps, the ramp rate dependence of the multipole fields is more accurately represented by half of the fitted slopes. Let $S_{\mathrm{A}_{\mathrm{n}}}$ and $S_{\mathrm{B}_{\mathrm{n}}}$ designate these half-slopes. Table IV summarizes the values of $S_{\mathrm{A}_{\mathfrak{n}}}$ and $S_{\mathrm{B}_{\mathfrak{n}}}$ for the measurements at the three positions.

In summary, it appears that the anomalous dynamic field behavior observed on the type-A magnets is ramp-rate related and that it ceases when the ramp is stopped. The amplitude and sign of the effect vary from multipole field to multipole field as well as along the magnet axis. However, for a given multipole field at a given position, the effect is independent of the current and increases quasi-linearly as a function of ramp rate. These observations, along with the ramp-induced heating brought to light by the quench 
performance and the special ramps, suggest that large and non-uniform eddy currents are the dominant cause of type-A behavior.

(a)

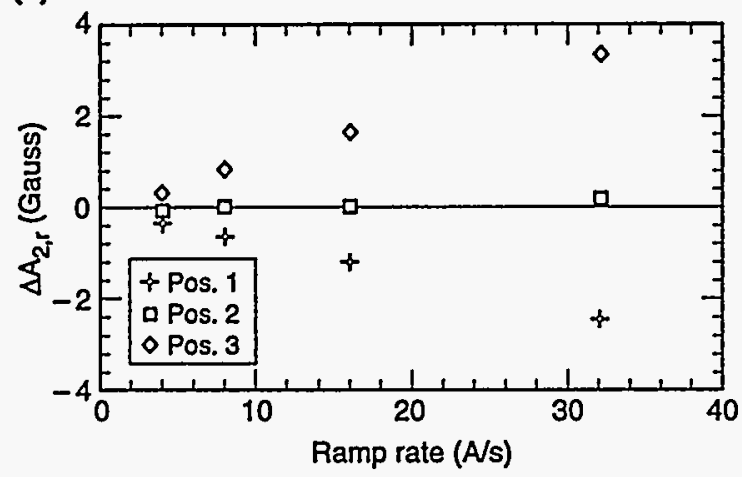

(b)

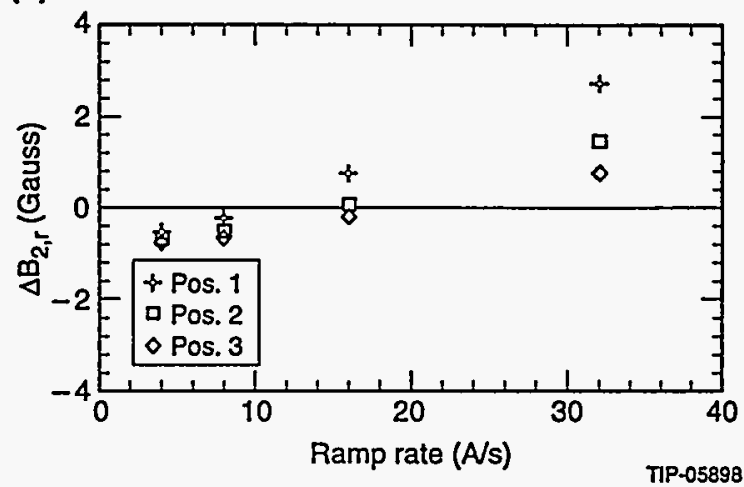

FIGURE 11: Width of re-scaled multipole field hystereses as a function of ramp rate for a set of magnetic measurements at three axial positions along magnet DCA312: a) skew sextupole field hysteresis width $\left(\triangle A_{2, \mathrm{r}}\right)$, and $\mathrm{b}$ ) normal sextupole field hysteresis width $\left(\Delta B_{2, \mathrm{r}}\right)$. The hystereses widths are expressed in Gauss and are calculated as the average differences between the up- and down-ramp re-scaled multipole fields for currents in the range $[2 \mathrm{kA}, 3 \mathrm{kA}]$. 
TABLE IV: Measured ramp rate dependence of multipole fields at three axial positions along the length of 5-cm aperture, 15-m long SSC dipole magnet prototype DCA312.a

Position $1 \quad$ Position $2 \quad$ Position 3

$\begin{array}{lccc}S_{\mathrm{A}_{1}}(\mathrm{Gauss} /(\mathrm{A} / \mathrm{s})) & -0.007 & -0.038 & 0.038 \\ \left.S_{\mathrm{B}_{1}}(\mathrm{Gauss} / \mathrm{A} / \mathrm{s})\right) & 0.034 & 0.042 & -0.052 \\ S_{\mathrm{A}_{2}}(\mathrm{Gauss} /(\mathrm{A} / \mathrm{s})) & -0.036 & 0.005 & 0.053 \\ \left.S_{\mathrm{B}_{2}}(\mathrm{Gauss} / \mathrm{A} / \mathrm{s})\right) & 0.060 & 0.040 & 0.028 \\ S_{\mathrm{A}_{3}}(\mathrm{Gauss} /(\mathrm{A} / \mathrm{s})) & -0.048 & -0.050 & -0.048 \\ \left.S_{\mathrm{B}_{3}}(\mathrm{Gauss} / \mathrm{A} / \mathrm{s})\right) & 0.020 & 0.013 & -0.005 \\ S_{\mathrm{A}_{4}}(\mathrm{Gauss} /(\mathrm{A} / \mathrm{s})) & -0.006 & 0.007 & 0.015 \\ S_{\mathrm{B}_{4}}(\mathrm{Gauss} /(\mathrm{A} / \mathrm{s})) & -0.024 & -0.029 & -0.038 \\ S_{\mathrm{A}_{5}}(\mathrm{Gauss} /(\mathrm{A} / \mathrm{s})) & -0.009 & -0.011 & -0.013 \\ S_{\mathrm{B}_{5}}(\mathrm{Gauss} /(\mathrm{A} / \mathrm{s})) & 0.003 & 0.000 & 0.000 \\ S_{\mathrm{A}_{6}}(\mathrm{Gauss} /(\mathrm{A} / \mathrm{s})) & -0.001 & 0.000 & 0.000 \\ S_{\mathrm{B}_{6}}(\mathrm{Gauss} /(\mathrm{A} / \mathrm{s})) & -0.004 & -0.004 & -0.005\end{array}$

${ }^{a}$ At the reference radius $(1 \mathrm{~cm})$. 


\subsection{Observation of periodic oscillations}

The multipole fields presented above were measured with either a $B$-series or a $F$-series mole, and, therefore, correspond to average values over a length of either $0.6 \mathrm{~m}$ or $1 \mathrm{~m}$.

It was reported recently that, when measured with a fine spatial resolution, the multipole fields of the HERA dipole magnets appeared to exhibit periodic oscillations about their mean values, with a wavelength equal to the pitch length of the inner-coil cable. ${ }^{33,36-38}$ Similar observations were made at $\mathrm{BNL}$ while measuring the sextupole fields of SSC dipole magnets using a three-Hall-probe array. ${ }^{39-41}$ The DESY measurements were taken at zero or constant transport-current and showed that the amplitude of these oscillations greatly depended on the excitation history. The three-Hall-probe measurements were taken both at constant transport-current and while ramping, and showed that, for most magnets, the amplitude of the oscillations varied as a function of current and ramp rate. One possible cause for these oscillations is thought to be imbalances in the current repartition among the cable strands. 42

In order to further investigate these phenomena, a series of measurements were taken on magnet DCA318 by means of a short mole developed by SSCL. The short mole is a modified $F$-series mole, with a 43 -mm long coil array comprising a tangential winding and the relevant bucking windings. The length of $43 \mathrm{~mm}$ was chosen because it corresponds to one half of the pitch length of the inner-coil cable. The test sequence for magnet DCA318 started with a cleansing quench and the usual pre-cycle. It was followed by a series of current loops up to $I_{\max }$ at rate $r_{0}$ during which measurements were taken. Between each of 
the loops, however, the short mole was moved along the magnet axis by a distance equal to $21.5 \mathrm{~mm}$.

Figures 12(a) through 12(d) present three-dimensional plots of the quadrupole and sextupole fields ( $z$-axis) as a function of position along the magnet axis ( $x$-axis) and current $(y$-axis) for a set of up-ramps from $115 \mathrm{~A}$ to $6000 \mathrm{~A}$ at $64 \mathrm{~A} / \mathrm{s}$. It appears clearly that the multipole fields oscillate as a function of axial position and that the amplitude of the oscillations increases as a function of current. It can also be verified that the wavelength of the oscillations is of the order of 4 measurement steps, i.e., $86 \mathrm{~mm}$, which corresponds to a full inner-coil cable pitch length. Similar observations can be made on most of the multipole fields of magnet DCA318. 


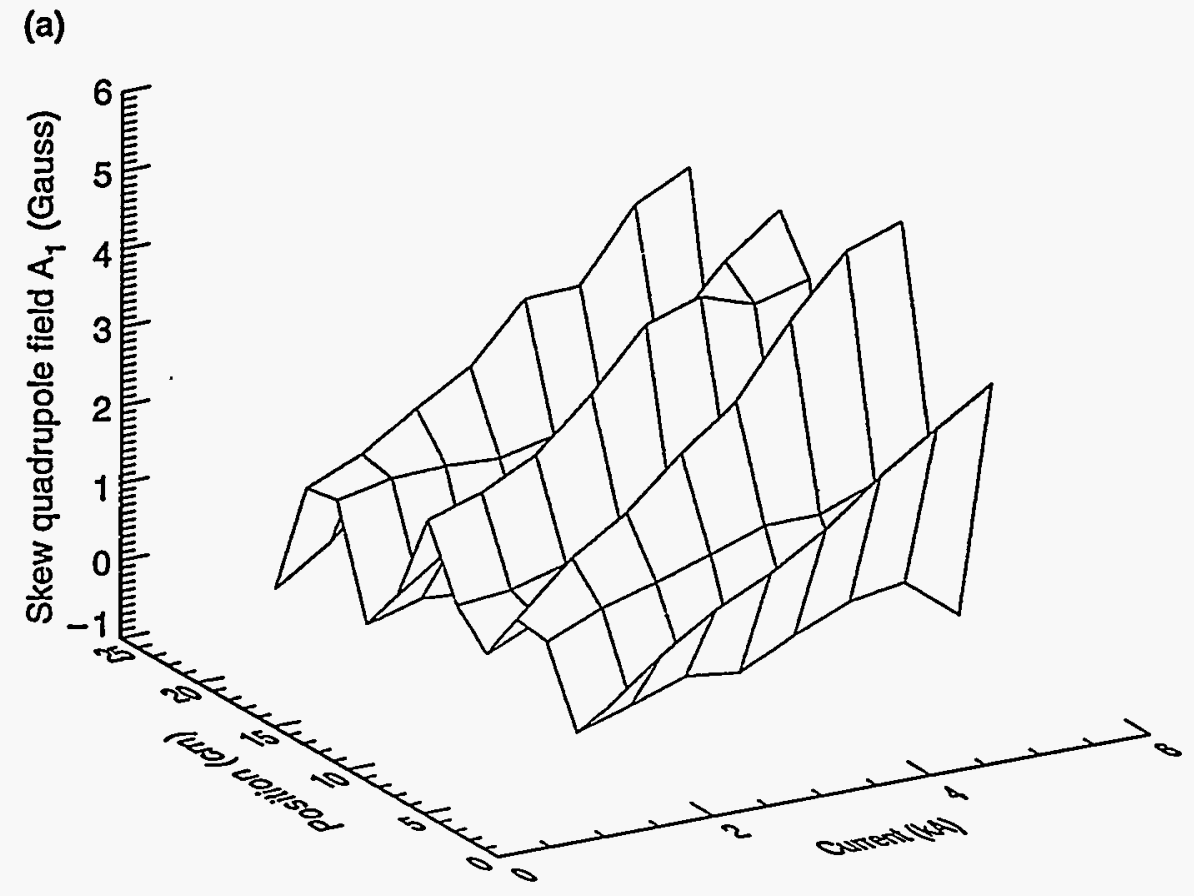

(b)

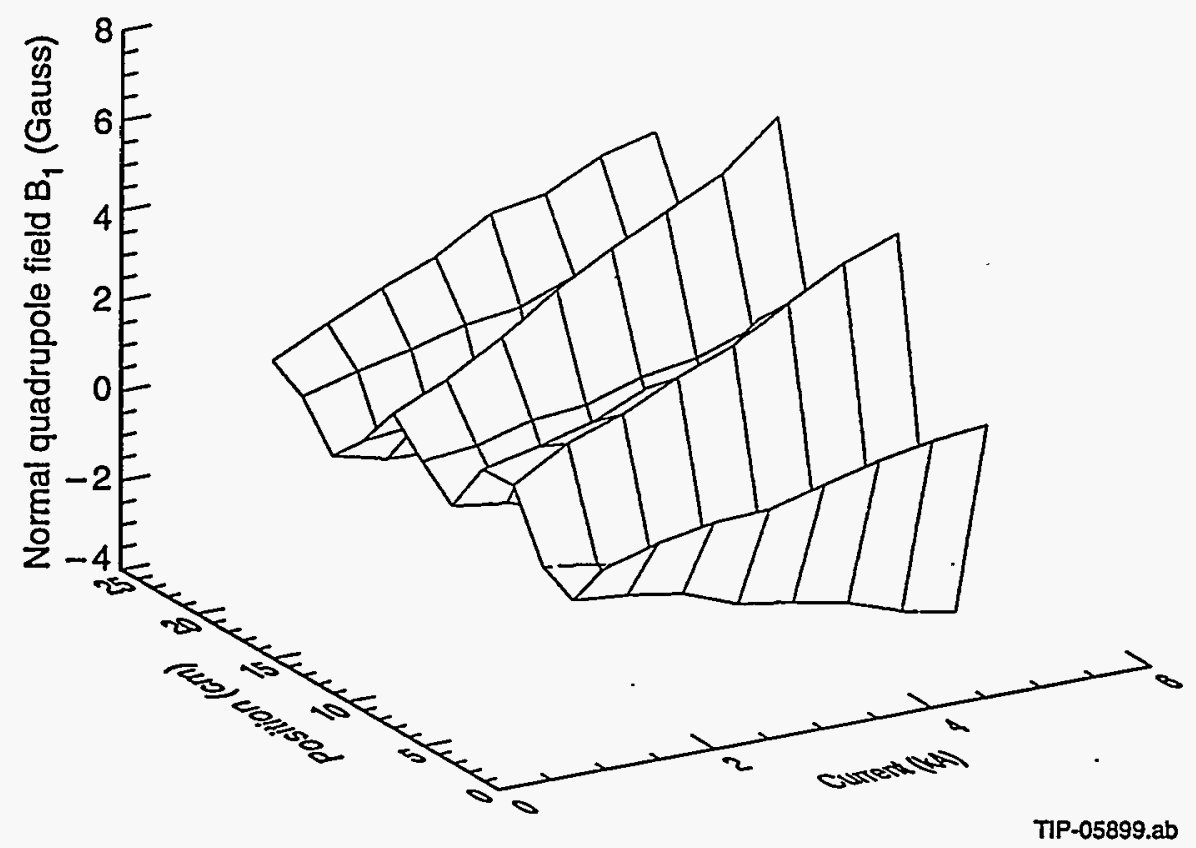

FIGURE 12: Multipole fields as a function of position and current as measured by a short mole during a series of current loops at $64 \mathrm{~A} / \mathrm{s}$ on magnet DCA318: a) skew quadrupole field, b) normal quadrupole field, c) skew sextupole field, and d) normal sextupole field. The multipole fields are in Gauss. The short mole length was $43 \mathrm{~mm}$. Between each of the current loops, the short mole was moved along the magnet axis by $21.5 \mathrm{~mm}$. 


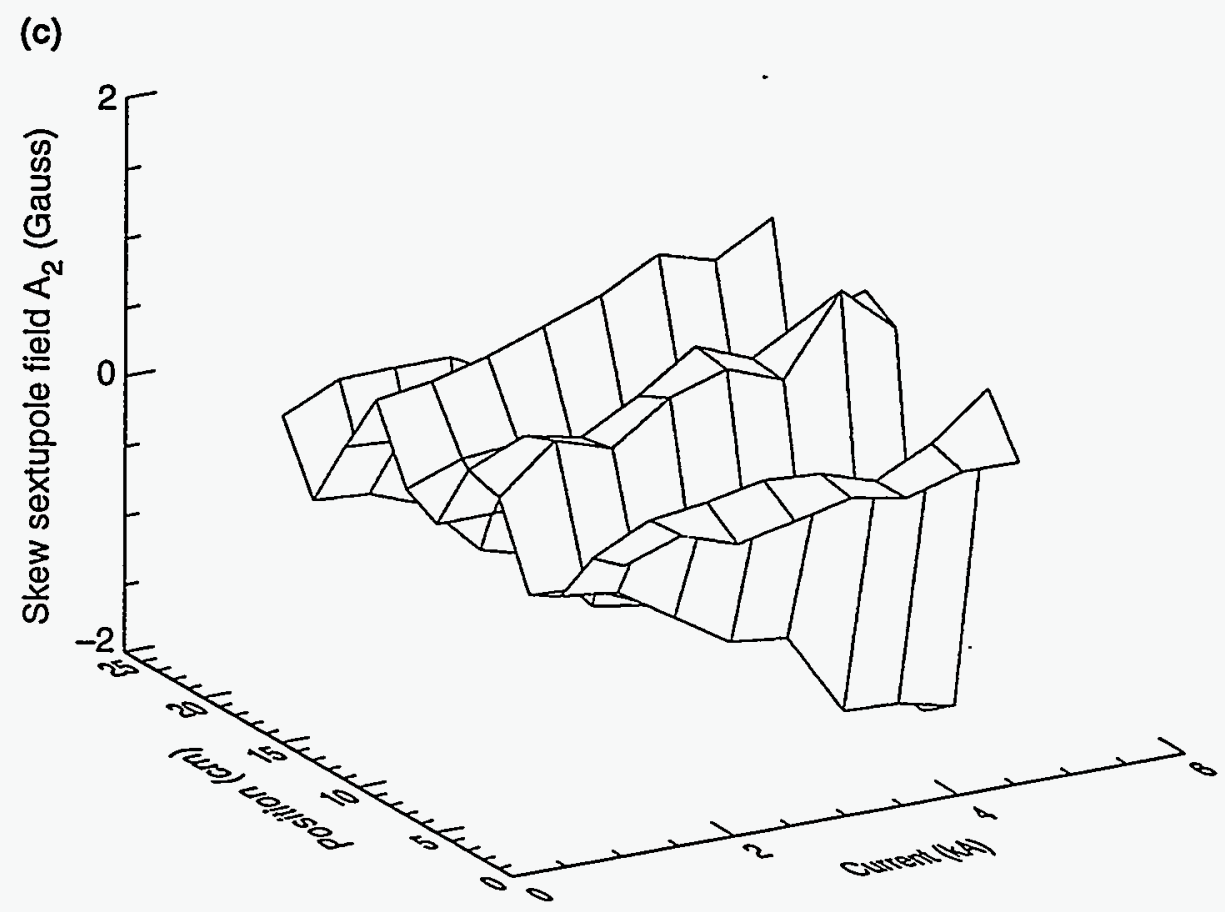

(d)

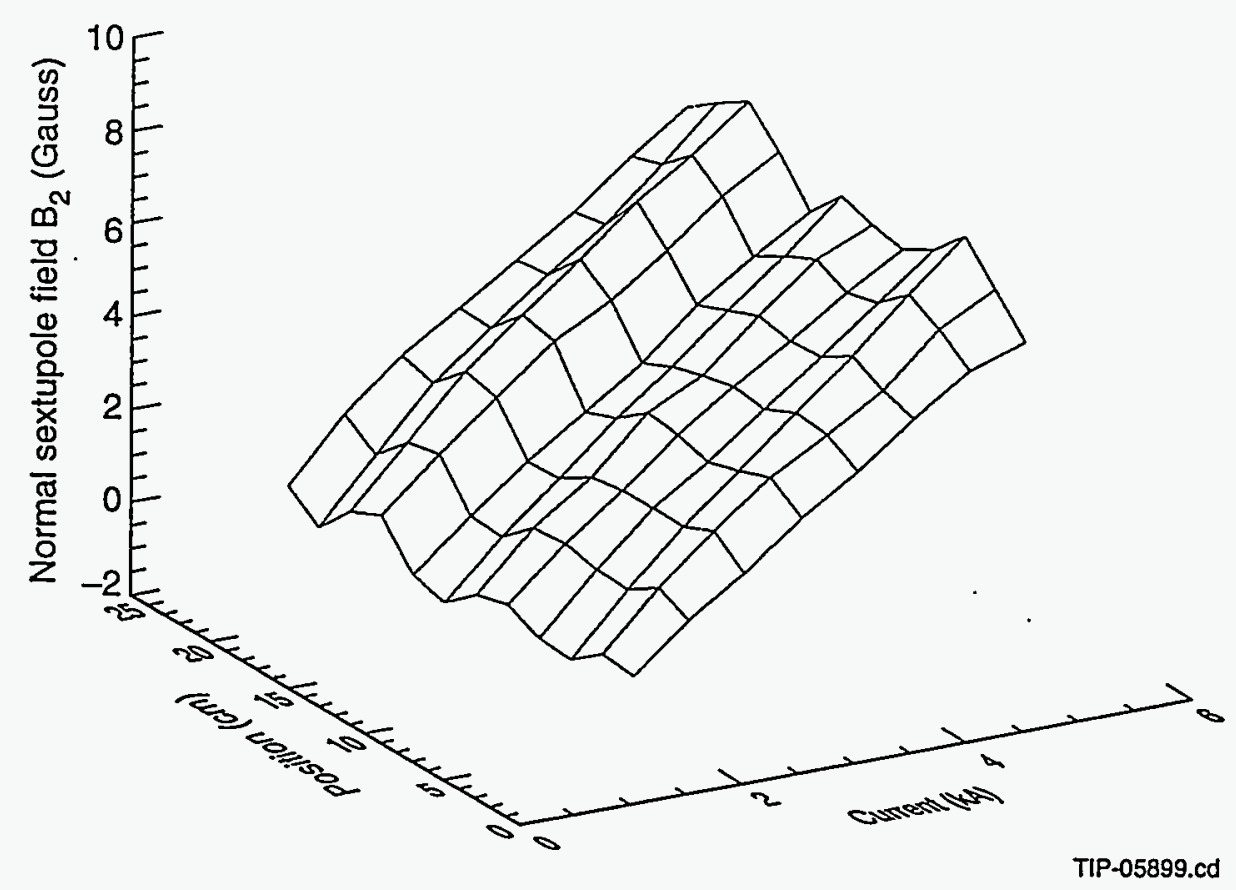

FIGURE 12: (Cont.) 
Superconducting magnets do not ordinarily dissipate energy when supplied with a constant current. However, a number of mechanisms that result in energy dissipation are known to occur when the current is cycled. Among them are losses due to the persistent magnetization currents generated in the superconductor, losses due to the magnetization of the iron yoke, and eddy-current losses. For a given current loop, the energy dissipated by the persistent magnetization currents and by the iron yoke magnetization is expected to be constant, independent of the ramp rate. The power dissipated by the eddy currents is expected to be proportional to the square of the ramp rate. For a given current cycle, the energy dissipated by the eddy currents is thus expected to increase linearly as a function of ramp rate. Measuring energy loss as a function of ramp rate thus provides a mean to assess the relative importance of eddy currents.

In addition to quench testing and magnetic measurements, energy loss measurements were performed on most of the magnets tested at $\mathrm{FNAL}^{43,44}$ and on a number of the magnets tested or retested at BNL. 45 The measurements were made electrically by means of digital multimeters sampling simultaneously the magnet voltage and current. The multimeters used at FNAL had a 7.5-digit resolution. They were configured to integrate the input reading over 10 power line cycles $(0.167 \mathrm{~s})$ and were triggered at a frequency of $4.5 \mathrm{~Hz}(0.222 \mathrm{~s})$. To compensate for the multimeter dead time (on the order of $0.056 \mathrm{~s}$ ), 4-Hz low-pass filters, with carefully matched time constants (on the order of $0.040 \mathrm{~s}$ ), were used on both the voltage and current inputs. The multimeters used at BNL had a 8.5-digit resolution. They were configured in order to maximize the number of data points taken, 
while keeping the integration time per data point equal to an integral number of power line cycles, $N_{\mathrm{L}}$. They were triggered at a frequency, $f$, defined as

$$
\frac{1}{f}=\left(\frac{N_{\mathrm{L}}}{f_{\mathrm{L}}}+0.002\right)
$$

where $f \mathrm{~L}$ is the power line frequency. $f \mathrm{~L}$ was measured internally, and $N_{\mathrm{L}}$ was varied from 2 to 10 . The dead time between two measurements was always $2 \mathrm{~ms}$.

A typical run consisted of a set of sawtooth ramps between $500 \mathrm{~A}$ and $5000 \mathrm{~A}$ with 5-s dwells at the minimum and maximum currents. The runs were repeated for a number of ramp rates in the range $16 \mathrm{~A} / \mathrm{s}$ to $250 \mathrm{~A} / \mathrm{s}$. For a given run at a given ramp rate, the energy loss was estimated by integrating numerically the product of the current and the voltage across the superconducting part of the magnet over a complete 500-5000-500 A cycle. For comparison, the energy dissipated by the persistent magnetization currents can be estimated using the DESY model mentioned earlier. For a 500-5000-500 A cycle at $4.35 \mathrm{~K}$, assuming that the inner (outer) strands contain 7250 (4200) filaments and that the superconductor critical current density is $3000 \mathrm{~A} / \mathrm{mm}^{2}$ at $4.22 \mathrm{~K}$ and $5 \mathrm{~T}$, we obtain: $40 \mathrm{~J} / \mathrm{m}$.

Figure 13(a) presents a summary plot of energy loss per cycle versus ramp rate, as measured on a number of FNAL-tested prototypes, while Fig. 13(b) presents a similar plot for some of the prototypes tested or retested at BNL. For each ramp rate, data were taken from a number of 500-5000-500-A cycles after at least three full cycles had been completed. For all the magnets measured at BNL and for magnets DCA312, DCA314, and DCA315 at FNAL, the energy loss per cycle appears to increase quasi-linearly as a function of ramp rate, as is expected from the effects of eddy currents. The intercepts and slopes of 
(a)

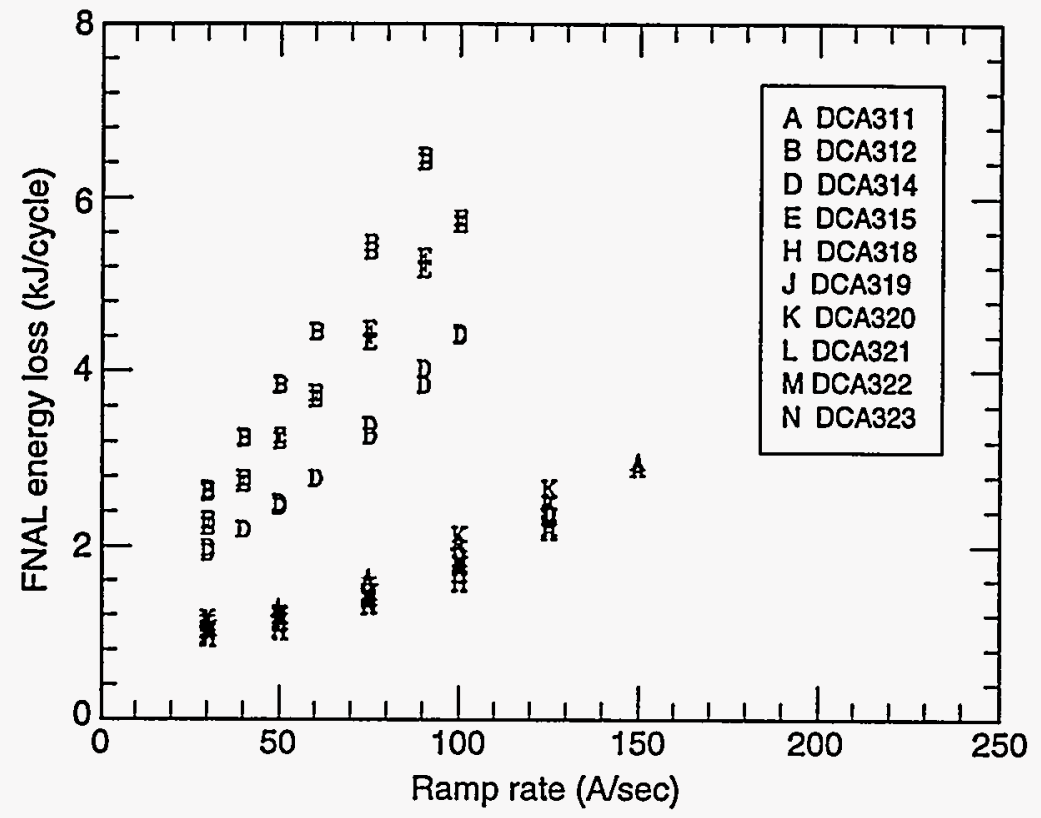

(b)

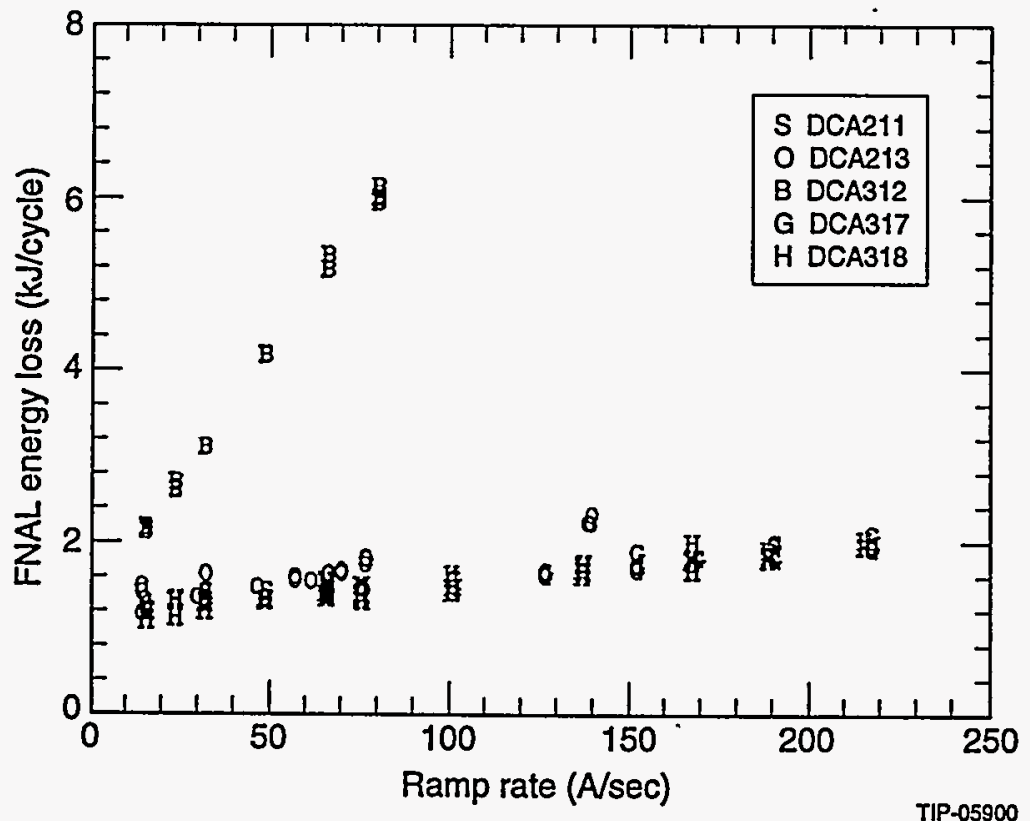

FIGURE 13: Energy loss for a 500-5000-500-A cycle versus ramp rate on selected 5-cm aperture, 15-m-long SSC dipole magnet prototypes: a) FNAL measurements and b) BNL measurements. 
first-order polynomial fittings of the data of Figs. 13(a) and 13(b) are listed in Tab. V. Figures 13(a) and 13(b) and Tab. V clearly show that the magnets with the largest losses are magnets DCA312, DCA315, and DCA314. These are the type-A magnets with the largest quench current degradation at high ramp rates and the most anomalous dynamic field behaviors. The energy loss data thus provide further evidences that the behavior of these magnets is eddy-current dominated. The BNL data also show that the magnets with the smallest losses are magnets DCA211, DCA317, and DCA318. Magnet DCA317 is the type-A magnet with the mildest quench current degradation. Magnet DCA318 is a typical type-B magnet, which exhibits a large drop of quench current at low ramp rates followed by a much milder degradation at larger rates. Also, in the range of investigation, the multipole fields of neither magnet DCA317 nor magnet DCA318 show a strong sensitivity to the ramp rate. The energy loss data thus confirm that eddy currents play little role in the behavior of these magnets.

Going back to Fig. 13(a), it appears that the energy losses of magnet DCA311 and of magnets DCA318 through DCA323 are very similar and increase somewhat quadratically as a function of ramp rate. This quadratic behavior, however, did not repeat when magnet DCA318 was re-measured at BNL, where the energy loss data reverted to a more conventional linear dependence. Table $\mathrm{V}$ also shows that the BNL-measured magnets have similar intercepts, of the order of $1200 \mathrm{~J}$. (Note that the energy loss data of magnet DCA211 are scarce and noisy). This is also the case for the three FNAL-measured magnets where a linear fitting is relevant (magnets DCA12, DCA314, and DCA315), except that their intercepts are of the order of $800 \mathrm{~J}$, thus about $30 \%$ lower than at BNL! These 
intercepts should correspond to the losses which are not ramp-rate dependent, i.e., the losses due to superconductor and iron magnetization. For magnets using cables made from similar strands and yoke laminations stamped from similar low carbon steel, the superconductor and iron magnetization losses, and thus, the intercepts, are expected to be reproducible.

TABLE V: Linear fitting parameters of the energy-loss data of Figs. 13(a) and 13(b) and estimated crossover resistances.

\begin{tabular}{|c|c|c|c|c|}
\hline $\begin{array}{l}\text { Magnet } \\
\text { Name }\end{array}$ & $\begin{array}{c}\text { Test } \\
\text { Facility }\end{array}$ & $\begin{array}{c}\text { Intercept } \\
\text { (J) }\end{array}$ & $\begin{array}{c}\text { Slope } \\
(\mathrm{J} /(\mathrm{A} / \mathrm{s}))\end{array}$ & $\begin{array}{c}\text { X-over } \\
\text { Res. } \\
(\mu \Omega)\end{array}$ \\
\hline DCA211 & $\mathrm{BNL}^{\mathrm{a}}$ & 1310 & 2 & $>160$ \\
\hline DCA213 & BNL & 1160 & 8 & $>40$ \\
\hline DCA311 & FNAL ${ }^{b}$ & 610 & 16 & \\
\hline \multirow[t]{2}{*}{ DCA312 } & FNAL & 710 & 64 & 5 \\
\hline & BNL & 1170 & 61 & 5 \\
\hline DCA314 & FNAL & 770 & 36 & 9 \\
\hline DCA315 & FNAL & 770 & 50 & 6 \\
\hline DCA317 & $\mathrm{BNL}$ & 1150 & 4 & $>80$ \\
\hline \multirow[t]{2}{*}{ DCA318 } & FNALb & 480 & 13 & \\
\hline & BNL & 1120 & 4 & $>80$ \\
\hline DCA319 & FNAL $^{b}$ & 470 & 14 & \\
\hline DCA320 & FNAL & 520 & 16 & \\
\hline DCA321 & FNAL $^{b}$ & 540 & 14 & \\
\hline DCA322 & FNAL $^{\mathrm{b}}$ & 530 & 14 & \\
\hline DCA323 & FNAL $^{b}$ & 600 & 11 & \\
\hline
\end{tabular}

a The data are noisy.

b The data appear to increase quadratically. 
The origin of the discrepancies between the FNAL and BNL measurements is not understood, rendering most interpretations of the energy loss data ${ }^{43}$ somewhat arguable. It is worth mentioning, however, that FNAL also built and tested a number of 5-cm aperture, 1.5-m long SSC dipole magnet models. For all the models which were measured, the energy loss appears to increase quasi-linearly as a function of ramp rate, with an estimated intercept of $100 \mathrm{~J} .{ }^{44}$ Considering that the ratio of superconductor and iron volume in long to short magnets is 10 while the ratio of magnetic lengths is 11 , this number scales advantageously to a value close to estimated intercepts of the BNL-measured long magnets.

Although the above observations partly discredit the energy loss data, it can still be noted that, for the magnet with the largest losses (magnet DCA312), the slope of the BNL data $(61 \mathrm{~J} /(\mathrm{A} / \mathrm{s}))$ is comparable to the slope of the FNAL data $(64 \mathrm{~J} /(\mathrm{A} / \mathrm{s}))$. The measured ramp-rate dependence of magnet $\mathrm{DCA} 312$ at $\mathrm{BNL}$ is thus similar to that measured at FNAL, and it is probably safe to extrapolate that the same would have been true for magnets DCA314 and DCA315. We therefore contend that the fitted slopes of magnets DCA312, DCA314, and DCA315 can be used for quantitative analyses, while the other magnets can be described as having smaller slopes than these three magnets. In the following, we shall refer to this slope as $S_{\mathrm{E}}$. 


\section{INFLUENCE OF EDDY CURRENTS}

\subsection{Eddy current sources}

All the data preceding suggest that the behavior of the type-A magnet is dominated by eddy currents. Let us start by reviewing the possible sources of eddy currents in a SSC-type magnet.

As we described earlier, the SSC magnet coils are wound with Rutherford-type conductors, which consist of a few tens of strands twisted together and shaped into a flat, two-layer, slightly keystoned cable. The strands themselves consist of thousands of superconducting filaments twisted together and embedded in a matrix of high purity copper. At liquid helium temperature, the resistivity of high purity copper becomes very low, eventually resulting in filament coupling. 46 Although the twisting of the filaments helps to reduce this effect, noticeable eddy currents flowing from one filament to another through the copper matrix can still be generated when the strands are subjected to a varying field. These eddy currents are referred to as intra-strand eddy currents.

Furthermore, the mid-thickness of the two-layer cable is smaller than twice the strand diameter and the contact surfaces at the crossovers between the strands of the two layers can be relatively large. Also, during magnet assembly, the coils are pre-compressed azimuthally. ${ }^{18}$ Large pressures are thus applied perpendicularly to the cables that keep the strands firmly in contact. The large contact surfaces and high pressures eventually result in low contact resistances at the strand crossovers that couple the cable strands. Loops are thus formed where significant eddy currents can take place when the cable is subjected to a varying field. These eddy currents are referred to as inter-strand eddy currents. 
In addition, eddy currents are generated in all the other conductive components of the magnet, such as copper wedges and iron yoke laminations, but these can be shown to only provide minor contributions. 47

This brief review thus points toward the cable and the cable strands as the largest sources of eddy currents. We shall now develop numerical models enabling one to compute the intra-strand and inter-strand eddy currents generated within a magnet coil along with the power they dissipate and the effects they produce on the multipole fields. These models, however, require a computation of the transport-current field. We thus shall begin by describing how to compute the transport-current field.

\subsection{Computing the transport-current field}

The transport-current field, $\mathbf{B}_{\mathfrak{t}}$, produced by the coil assembly of a dipole magnet like those pictured in Figs. 1(a) and 1(b) can be calculated by dividing each turn of the coil into elementary current-lines parallel to the $z$-axis.

Let $l$ be an index referring to the coil layer number, $k$ be an index referring to the turn number within a given coil layer, and $j$ be an index referring to the current-line number within a given turn. From Ampere's theorem, the field, $\mathbf{B}_{\mathbf{t}}^{\mathbf{l}, \mathbf{k}, \mathbf{j}}(x+\mathrm{i} y)$, generated by a given current-line is

$$
\mathrm{B}_{t}^{\mathrm{l}, \mathrm{k}, \mathbf{j}}(x+\mathrm{i} y)=\frac{\mu_{0} I_{t}^{1, k, j}}{2 \pi\left((x+\mathrm{i} y)-\mathbf{z}_{\mathbf{t}}^{\mathrm{l}, \mathrm{k}, \mathbf{j}}\right)},
$$


where $I_{1, k, j}$ is the current-line intensity and $z_{t}^{1, k, j}=x_{t}^{1, k, j}+i y_{t}^{1, k, j}$ is the current-line position in the complex plane. The current-line intensity is related to the transport-current intensity, $I_{t}$, by

$$
I_{\mathrm{t}}^{1, \mathrm{k}, \mathrm{j}}=\varepsilon_{\mathrm{l}, \mathrm{k}} \frac{I_{\mathrm{t}}}{n_{\mathrm{l}, \mathrm{k}}}
$$

where $n_{1, \mathrm{k}}$ is the number of current-lines used to represent turn $k$ of layer $l, \varepsilon_{1, \mathrm{k}}=-1$ for a current-line in quadrant 1 or $4\left(\operatorname{Re}\left(\mathbf{z}_{t}^{1, k, j}\right)>0\right)$, and $\varepsilon_{l, k}=+1$ for a current-line in quadrant 2 or $3\left(\operatorname{Re}\left(\mathbf{z}_{t}^{1, k, j}\right)<0\right)$.

If the current-line is located inside a circular iron yoke of radius, $R_{\mathrm{y}}$, the contribution of the iron yoke can be shown to be the same as that of a mirror current-line of intensity, $I_{t, m}^{l, k, j}$, and position, $\mathbf{z}_{\mathbf{t}, \mathbf{m}}^{\mathbf{l}, \mathbf{k}, \mathbf{j}}$, where 33

$$
I_{\mathrm{t}, \mathrm{m}}^{1, \mathrm{k}, \mathrm{j}}=\frac{\mu-1}{\mu+1} I_{t}^{1, \mathrm{k}, \mathrm{j}}
$$

and

$$
\mathbf{z}_{t, m}^{1, k_{j} \mathbf{j}}=\frac{R_{y}{ }^{2}}{\left(\mathbf{z}_{t}^{1, k_{\mathbf{y}}}\right)^{*}}
$$

Here $\mu$ designates the magnetic permeability of the iron yoke and $\left(\mathbf{z}_{t}^{1, k_{j} \mathbf{j}}\right)^{*}$ designates the complex conjugate of $\mathbf{z}_{\mathbf{t}}^{\mathbf{l}, \mathbf{k}, \mathbf{j}}$. Note that the mirror image method is only applicable if the iron yoke permeability is uniform.

The transport-current field produced by the magnet assembly is obtained by summing the contributions of all the elementary current lines. In the current range where the iron yoke is not saturated, $\mathbf{B}_{\mathbf{t}}$ may thus be written

$$
\mathrm{B}_{\mathrm{t}}(x+\mathrm{i} y)=I_{\mathbf{t}} \mathrm{T}_{\mathbf{t}}(x+\mathrm{i} y)
$$


where $\mathbf{T}_{\mathbf{t}}$ is the local transfer function given by

$$
\mathrm{T}_{\mathbf{t}}(x+\mathrm{i} y)=\frac{\mu_{0}}{2 \pi} \sum_{l=1}^{2} \sum_{k=1}^{K_{1}} \frac{\varepsilon_{\mathrm{l}, \mathrm{k}}}{n_{\mathrm{l}, \mathrm{k}}} \sum_{j=1}^{n_{1, \mathrm{k}}}\left[\frac{1}{(x+\mathrm{i} y)-\mathbf{z}_{\mathbf{t}}^{\mathbf{l}, \mathbf{k}, \mathbf{j}}}+\frac{\mu-1}{\mu+1} \frac{1}{(x+\mathrm{i} y)-\mathbf{z}_{\mathbf{t}, \mathbf{m}}^{\mathbf{l}, \mathbf{k}, \mathbf{j}}}\right] .
$$

Here, $K_{1}$ designates the total number of turns in layer $l$. Eq. (11) shows that, in the current range where the iron yoke is not saturated, $T_{t}$ is independent of the current and is only determined by coil geometry and by the inner radius and the magnetic permeability of the iron yoke. (In most practical cases, $\mu$ can be assumed to be infinite.)

For $|x+i y|<,\left|z_{t}^{1, k, j}\right|$, the first fraction of Eq. (11) can be expanded as a series

$$
\frac{1}{(x+\mathrm{i} y)-\mathbf{z}_{\mathbf{t}}^{1, k, \mathbf{j}} \mathbf{j}}=-\frac{1}{\mathrm{z}_{\mathrm{t}}^{1, \mathbf{k}, \mathbf{j}}} \frac{1}{1-\frac{x+\mathrm{i} y}{\mathrm{z}_{\mathrm{t}}^{1, k, j}}}=-\frac{1}{\mathrm{z}_{\mathrm{t}}^{1, \mathbf{k}, \mathbf{j}}} \sum_{n=0}^{\infty}\left(\frac{x+\mathrm{i} y}{\mathrm{z}_{\mathrm{t}}^{1, \mathbf{k}, \mathbf{j}}}\right)^{\mathrm{n}}
$$

and, for $|x+i y|<\mid z_{t, m}^{1, k, j}$, , a similar series expansion can be derived for the second fraction of Eq. (11).

Hence, within the coil aperture, $B_{t}$ may be written

$$
\mathbf{B}_{\mathrm{t}}(x+\mathrm{i} y)=\sum_{n=0}^{\infty}\left(B_{\mathrm{n}, \mathrm{t}}+\mathrm{i} A_{\mathrm{n}, \mathrm{t}}\left(\frac{x+\mathrm{i} y}{R_{\mathrm{ref}}}\right)^{\mathrm{n}}\right.
$$

where $R_{\text {ref }}$ is the reference radius introduced in Eq. (2), and $A_{\mathrm{n}, \mathrm{t}}$ and $B_{\mathrm{n}, \mathrm{t}}$ are the geometric components of the $(2 n+1)$-pole fields given by

$$
B_{\mathrm{n}, \mathrm{t}}+\mathrm{i} A_{\mathrm{n}, \mathrm{t}}=-\frac{\mu_{0} I_{\mathrm{t}}}{2 \pi R_{\mathrm{ref}}} \sum_{\mathrm{l}=1}^{2} \sum_{\mathrm{k}=1}^{\mathrm{K}_{1}} \frac{\varepsilon_{\mathrm{l}, \mathrm{k}}}{\mathrm{n}_{1, \mathrm{k}}} \sum_{\mathrm{j}=1}^{\mathrm{n}_{1, \mathrm{k}}}\left[\left(\frac{R_{\mathrm{ref}}}{\mathbf{z}_{\mathrm{t}}^{1, k, j}}\right)^{\mathrm{n}+1}+\frac{\mu-1}{\mu+1}\left(\frac{R_{\mathrm{ref}}}{\mathbf{z}_{\mathrm{t}, \mathrm{m}}, \mathbf{k}, \mathbf{j}}\right)^{\mathrm{n}+1}\right] .
$$

Equation (14) shows that $A_{\mathrm{n}, \mathrm{t}}$ and $B_{\mathrm{n}, \mathrm{t}}$ varies linearly as a function of $I_{\mathrm{t}}$. 
In practice, a good computational accuracy can be achieved by taking for $n_{1, k}$ an even number of the order of $N_{1}$, where $N_{1}$ is the number of strands in the layer-l cable, and by representing each turn by two layers of equally spaced current-lines (see Fig. 14). In this paper, we use

$$
n_{1, \mathrm{k}}=N_{\mathrm{l}}, \quad \text { for } N_{\mathrm{l}} \text { even, }
$$

and

$$
n_{\mathrm{l}, \mathrm{k}}=N_{\mathrm{l}}-1, \quad \text { for } N_{\mathrm{l}} \text { odd, }
$$

and we take for $\mathbf{z}_{t}^{1, k, j}$

$\mathbf{z}_{\mathrm{t}}^{1, \mathrm{k}_{\mathrm{j}}}=\left(1-\frac{2 j-1}{n \mathrm{l}, \mathrm{k}}\right)\left(\frac{3 \mathbf{Z}_{1}^{\mathrm{l}, \mathrm{k}}+\mathbf{Z}_{2}^{1, \mathrm{k}}}{4}\right)+\frac{2 j-1}{n \mathrm{l}, \mathrm{k}}\left(\frac{\mathbf{z}_{3}^{\mathrm{l}, \mathrm{k}}+3 \mathbf{Z}_{4}^{\mathrm{l}, \mathrm{k}}}{4}\right), \quad$ for $j, 1 \leq j \leq \frac{n \mathrm{l}, \mathrm{k}}{2}$,

and

$$
\begin{array}{r}
\mathbf{z}_{\mathbf{t}}^{1, k, j}=\left(1-\frac{2 j-n l, k-1}{n l, k}\right)\left(\frac{\mathbf{Z}_{1}^{\mathrm{l}, \mathrm{k}}+3 \mathbf{Z}_{2}^{\mathrm{l}, \mathrm{k}}}{4}\right)+\frac{2 j-n l, \mathrm{k}-1}{n l, \mathrm{k}}\left(\frac{3 \mathbf{Z}_{3}^{\mathrm{l}, \mathrm{k}}+\mathbf{Z}_{4}^{\mathrm{l}, \mathrm{k}}}{4}\right), \\
\quad \text { for } j, \frac{n 1, \mathrm{k}}{2}+1 \leq j \leq n 1, \mathrm{k},
\end{array}
$$

where $\mathbf{Z}_{1}^{1, k}$ through $\mathbf{Z}_{4}^{1, k}$ designate the positions in the complex plane of the four corners of turn $k$ of layer $l$ as defined in Fig. 14 . 


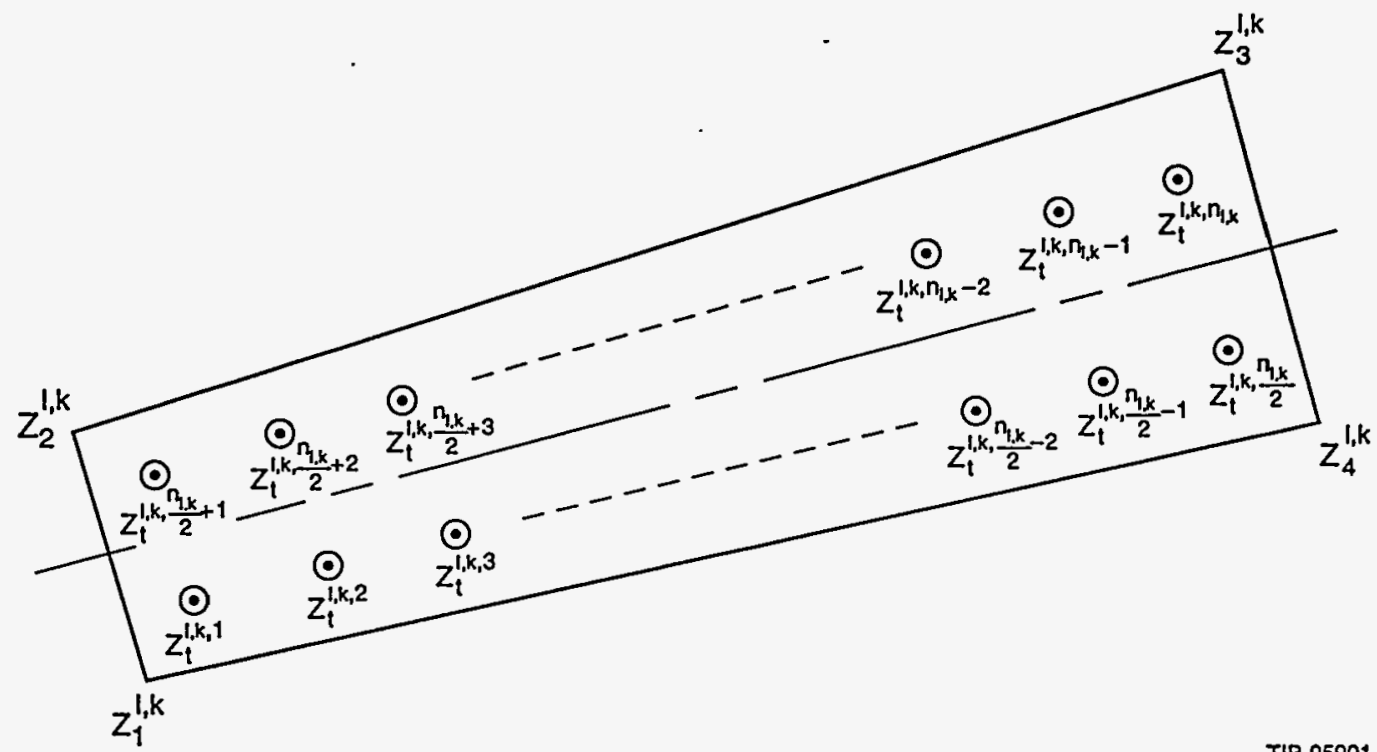

FIGURE 14: Current-line model for the calculation of the transport-current field produced by a given turn of a two-layer cosine-theta coil wound with Rutherford-type cables.

\subsection{Model for intra-strand eddy currents}

7.3.1. Case of a single strand Let us first consider a rectilinear and infinite strand, exposed to a uniform, time-dependent external field, $\mathbf{B}_{t}(t)$, perpendicular to its axis. The shielding currents generated within the strand can be shown to produce a magnetic moment, $\mathbf{M}_{\mathbf{S}}$, given by 46

$$
\mathbf{M}_{\mathrm{s}}=M_{\mathrm{sy}}+\mathrm{i} M_{\mathrm{sx}}=-\frac{2 \pi R_{3}^{2}}{\mu_{0}} \frac{\mathrm{d} \mathbf{B}_{\mathrm{t}}}{\mathrm{d} t} \tau_{\mathrm{s}}
$$

where $M_{\mathrm{sx}}$ and $M_{\mathrm{sy}}$ are the $x$ - and $y$-components of the magnetic moment, $\mu_{0}$ is the permeability of vacuum, $R_{3}$ is the strand radius, and $\tau_{\mathrm{S}}$ is the effective time constant of the shielding currents. The power, $P_{\mathrm{S}}$, dissipated per strand unit length by the shielding currents is given by 


$$
P_{\mathrm{s}}=\frac{2 \pi R_{3}^{2}}{\mu_{0}}\left|\frac{\mathrm{dB}}{\mathrm{dt}}\right|^{2} \tau_{\mathrm{s}}
$$

As can be seen from the micrograph presented in Fig. 15, the SSC strands consist of three concentric regions: 1 ) a copper inner core, of outer radius, $R_{1}$, and resistivity, $\rho_{1}$, 2) an annular multifilamentary composite, of outer radius, $R_{2}$, and transverse resistivity, $\rho_{2}$, and 3) a copper outer sheath, of resistivity, $\rho_{3}$. For such geometry, the effective time constant of the shielding currents can be estimated as ${ }^{48}$

$$
\tau_{\mathrm{s}}=\tau_{\text {core }}+\tau_{\text {composite }}+\tau_{\text {sheath }}
$$

where $\tau_{\text {core }}$ corresponds to the shielding currents generated in the inner core

$$
\tau_{\text {core }}=\frac{\mu_{0}}{2 \rho_{1}} \frac{R_{1}^{2}}{R_{3}^{2}}\left[1+\left(\frac{\pi}{L_{s}}\right)^{2} R_{1}^{2}\right]\left(\frac{L_{s}}{2 \pi}\right)^{2},
$$

$\tau_{\text {composite }}$ corresponds to the shielding currents generated in the annular multifilamentary composite

$$
\tau_{\text {composite }}=\frac{\mu_{0}}{2 \rho_{2}} \frac{R_{2}^{2}-R_{1}^{2}}{R_{3}^{2}}\left[1+\left(\frac{\pi}{L_{\mathrm{s}}}\right)^{2}\left(R_{1}^{2}+R_{2}^{2}\right)\right]\left(\frac{L_{\mathrm{s}}}{2 \pi}\right)^{2}
$$

and $\tau_{\text {sheath }}$ corresponds to the shielding currents generated in the outer sheath

$$
\tau_{\text {sheath }}=\frac{\mu_{0}}{2 \rho_{3}} \frac{R_{3}{ }^{2-} R_{2} 2}{R_{3}{ }^{2}}\left[\frac{R_{2}^{2}}{R_{2}{ }^{2}+R_{3}{ }^{2}}+\left(\frac{\pi}{L_{\mathrm{s}}}\right)^{2}\left(R_{2}{ }^{2}+R_{3}{ }^{2}\right)\right]\left(\frac{L_{\mathrm{s}}}{2 \pi}\right)^{2} .
$$

In Eqs. (20a) through (20c), $L_{s}$ designates the strand twist pitch. 


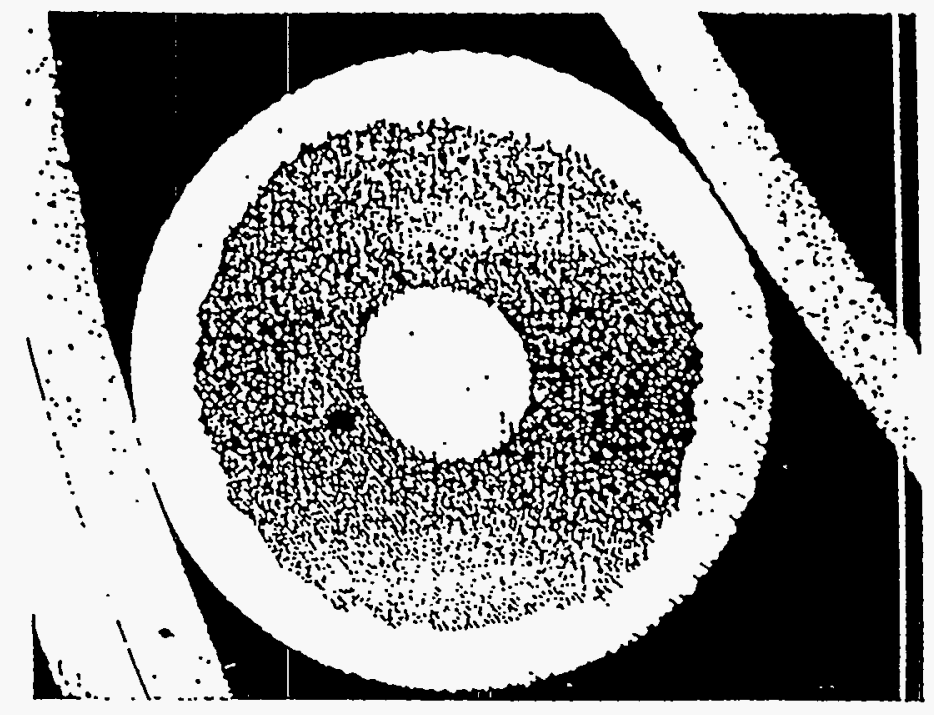

FIGURE 15: Micrograph of a SSC inner strand. The strand consists of three concentric regions: 1) a copper inner core, 2) an annular superconducting multifilamentrary composite, and 3) a copper outer sheath.

The resistivity of the inner core and of the outer sheath can be taken equal to the resistivity of bulk copper, $\rho_{\mathrm{b}}$, which depends on the bulk copper RRR, noted $R R R_{b}$, the local temperature, $T$, and the local field strength, $B_{\mathrm{t}}$. For the case of high contact resistance between the superconducting filaments and the copper matrix, the transverse resistivity of the multifilamentary composite can be evaluated as 49

$$
\rho_{2}=\frac{1+\lambda}{1-\lambda} \rho_{\mathrm{m}}
$$

where $\lambda$ is the fraction of superconductor in the composite and $\rho_{\mathrm{m}}$ is the copper matrix resistivity. 
For an hexagonal lattice of filaments, we have

$$
\lambda=\frac{\pi}{2 \sqrt{3}}\left(1+\frac{s}{d}\right)^{-2}
$$

where $d$ is the filament diameter and $s$ is the filament spacing. In the case of SSC strands, $d=6 \mu \mathrm{m}, s=1 \mu \mathrm{m}$, and $\lambda \approx 0.67$

In the case of SSC strands, the determination of $\rho_{\mathrm{m}}$ is complicated by the fact that, at low temperatures, the interfilament spacing is smaller than the electron mean free path in copper. Hence, at low temperatures, the filaments of the composite act as boundary scatterers, resulting in an enhancement of the copper matrix resistivity with respect to bulk copper. This enhancement can be estimated as 50

$$
\rho_{\mathrm{m}}=\rho_{\mathrm{b}}+\frac{6.5610^{-16}}{s}
$$

For $R_{R R}=200$, we get: $\rho_{\mathrm{b}}(10 \mathrm{~K}, 0 \mathrm{~T})=7.810^{-11} \Omega \mathrm{m}$ and $\rho_{\mathrm{m}}(10 \mathrm{~K}, 0 \mathrm{~T})=$ 7.3 $10^{-10} \Omega \mathrm{m}$, which shows that, at low temperatures, the copper matrix can be 10 times more resistive than the inner core or the outer shell.

Furthermore, the RRR values quoted in Tab. I as measured on cable short samples or on magnets during cold testing correspond to

$$
\mathrm{RRR}=\frac{\rho_{\mathrm{t}}(273 \mathrm{~K}, 0 \mathrm{~T})}{\rho_{\mathrm{t}}(10 \mathrm{~K}, 0 \mathrm{~T})}
$$

where $\rho_{\mathrm{t}}$ is the longitudinal resistivity of the cable. For SSC-type cables 


$$
\frac{1}{\rho_{\mathrm{t}}} \approx\left(1-\frac{1}{\lambda} \frac{1}{1+r_{\mathrm{cus}}}\right) \frac{1}{\rho_{\mathrm{b}}}+\frac{1-\lambda}{\lambda} \frac{1}{1+r_{\mathrm{cus}}} \frac{1}{\rho_{\mathrm{m}}},
$$

where $r_{\text {cus }}$ is the strand copper-to-superconductor ratio.

At $273 \mathrm{~K}, \rho_{\mathrm{m}} \approx \rho_{\mathrm{b}}$, and Eq. (25) can be written

$$
\rho_{\mathrm{t}}(273 \mathrm{~K}, 0 \mathrm{~T}) \approx \frac{1+r_{\text {cus }}}{r_{\text {cus }}} \rho_{\mathrm{b}}(273 \mathrm{~K}, 0 \mathrm{~T}) .
$$

At $10 \mathrm{~K}$, however, one needs to take into account the enhancement of the copper matrix resistivity due to boundary scattering. Eq. (25) then becomes

$$
\frac{1}{\rho_{\mathrm{t}}(10 \mathrm{~K}, 0 \mathrm{~T})} \approx\left(1-\frac{1}{\lambda} \frac{1}{1+r_{\mathrm{cus}}}\right) \frac{1}{\rho_{\mathrm{b}}(10 \mathrm{~K}, 0 \mathrm{~T})}+\frac{1-\lambda}{\lambda} \frac{1}{1+r_{\mathrm{cus}}} \frac{1}{\rho_{\mathrm{b}}(10 \mathrm{~K}, 0 \mathrm{~T})+\frac{6.5610^{-16}}{s}} \text {. }
$$

By combining Eqs. (24), (26a), and (26b), and introducing the definition of $R R R_{b}$, we obtain

$$
\mathrm{RRR} \approx \frac{\lambda\left(1+r_{\text {cus }}\right)-1}{\lambda r_{\text {cus }}} \mathrm{RRR}_{\mathrm{b}}+\frac{1-\lambda}{\lambda r_{\text {cus }}} \frac{1}{\frac{1}{\mathrm{RRR}_{\mathrm{b}}}+\frac{4.2310^{-8}}{s}}
$$

Here we have assumed: $\rho_{\mathrm{b}}(273 \mathrm{~K}, 0 \mathrm{~T}) \approx 1.5510^{-8} \Omega \mathrm{m}$.

Equation (27) allows one to determine $R R R_{b}$ from the measured $R R R$ of the cable. As an illustration, for $R R R=200$ and $r_{\text {cus }}=1.5$, we get: $R R R_{b} \approx 290$, which corresponds to the RRR value of the raw copper thought to have been used by the strand manufacturers. ${ }^{23}$ Thus, for the magnets listed in Tab. I as having a RRR of the order of 200 , we can conclude that coil curing was accompanied by a nearly full annealing of the copper. 
Having calculated $\rho_{1}, \rho_{2}$, and $\rho_{3}$, we can now go back to the estimation of the effective time constant of the shielding currents. Let $x_{\text {core }}$ designate the ratio of the inner core cross-sectional area to the strand cross-sectional area. We have

$$
R_{1}=\sqrt{x_{\text {core }}} R_{3},
$$

and

$$
R_{2}=\sqrt{x_{\text {core }}+\frac{1}{\lambda\left(1+r_{\text {cus }}\right)}} R_{3}
$$

For SSC inner strands, $R_{3}=0.404 \mathrm{~mm}$ and $x_{\text {core }} \approx 10 \%$. With $r_{\text {cus }}=1.5$, we get: $R_{1} \approx$ $0.128 \mathrm{~mm}$ and $R_{2} \approx 0.338 \mathrm{~mm}$, and with $L_{\mathrm{s}}=13 \mathrm{~mm}$, Eqs. (20a) through (20c) become

$$
\begin{gathered}
\tau_{\text {core }}=\frac{2.6910^{-13}}{\rho_{\mathrm{b}}\left(\mathrm{RRR}_{\mathrm{b}}, T, B_{\mathrm{t}}\right)}, \\
\tau_{\text {composite }}=\frac{3.2610^{-13}}{\rho_{\mathrm{b}}\left(\mathrm{RRR}_{\mathrm{b}}, T, B_{\mathrm{f}}\right)+6.5610^{-10}},
\end{gathered}
$$

and

$$
\tau_{\text {sheath }}=\frac{3.4510^{-13}}{\rho_{\mathrm{b}}\left(\mathrm{RRR}_{\mathrm{b}}, T, B_{\mathrm{t}}\right)}
$$

These time constants can be compared to the time constants derived from ac loss measurements on strand short samples reported in Reference 51. For RRR $=37$ (sample F-1), $T=10 \mathrm{~K}$, and $B=1 \mathrm{~T}: \tau_{\text {core }}=0.8 \mathrm{~ms}, \tau_{\text {composite }}=0.3 \mathrm{~ms}$, and $\tau_{\text {sheath }}=1.0 \mathrm{~ms}$, which gives: $\tau_{\mathrm{s}}=2.1 \mathrm{~ms}$, while the ac loss measurements yielded $3.6 \mathrm{~ms}$. For $\mathrm{RRR}=126$ (sample $\mathrm{F}-2), T=10 \mathrm{~K}$, and $B=1 \mathrm{~T}: \tau_{\text {core }}=2.2 \mathrm{~ms}, \tau_{\text {composite }}=0.4 \mathrm{~ms}$, and $\tau_{\text {sheath }}=2.9 \mathrm{~ms}$, which gives: $\tau_{\mathrm{s}}=5.5 \mathrm{~ms}$, while the ac loss measurements yielded $6.5 \mathrm{~ms}$. The predicted values 
thus appear to be of the right order of magnitude, but somewhat lower than the values derived from the test data.

7.3.2 Case of a magnet coil Having treated the case of a rectilinear and infinite strand, we can now go back to the case of a magnet coil. In the current-line model described in section 7.2, the effects of intra-stand eddy currents can be calculated by associating with each current-line a magnetic moment, $\mathbf{M}_{\mathbf{i n t r a}}^{\mathbf{l}, \mathbf{k}, \mathbf{j}}$, defined as

$$
\mathbf{M}_{\text {intra }}^{1, \mathbf{k}, \mathbf{j}}=-\frac{N_{\mathrm{l}}}{n_{\mathrm{l}, \mathrm{k}}} \frac{2 \pi\left(R_{3}^{1}\right)^{2}}{\mu_{0}} \frac{\mathrm{dB}_{\mathrm{t}}\left(\mathrm{z}_{\mathrm{t}}^{1, \mathrm{k}, \mathbf{j}}\right)}{\mathrm{dt}} \tau_{\mathrm{s}}^{1, \mathrm{k}, \mathrm{j}}
$$

where $R_{3}^{l}$ designates the outer radius of the strands of the layer-l cable, and $\tau_{\mathrm{s}}^{1, \mathrm{k}, \mathrm{j}}$ designates the effective time constant of the shielding currents estimated for a strand of the layer- $l$ cable at a field of $B_{\mathrm{t}}\left(\mathrm{z}_{\mathbf{t}}^{1, \mathbf{k}, \mathbf{j}}\right)=\left|\mathbf{B}_{\mathbf{t}}\left(\mathrm{z}_{\mathbf{t}}^{1, k, \mathbf{j}}\right)\right|$. When ramping the transport-current, the time derivative of $\mathbf{B}_{\mathfrak{t}}$ is simply given by

$$
\frac{\mathrm{d} \mathbf{B}_{t}\left(\mathbf{z}_{t}^{1, k, j}\right)}{\mathrm{dt}}=\frac{\mathrm{d} I_{t}}{\mathrm{~d} t} T_{t}\left(\mathbf{z}_{t}^{1, k, j}\right)
$$

The field, $\mathbf{B}_{\mathbf{i} \text { tra }}^{\mathbf{l}, \mathbf{k}, \mathbf{j}}$, produced by $\mathbf{M}_{\mathbf{i n t r a}}^{\mathbf{l}, \mathbf{k}, \mathbf{j}}$ may be derived by representing the magnetic moment by the current-line doublet shown in Fig. 16. This doublet consists of a current-line of intensity $\left(-I_{\mathrm{d}}^{1, \mathrm{k}, \mathrm{j}}\right)$ located at $\mathbf{z}_{\mathbf{t}}^{1, \mathrm{k}, \mathrm{j}}$, and a current-line of intensity $\left(+I_{\mathrm{d}}^{1, \mathrm{k}, \mathrm{j}}\right)$ located at $\left(z_{t}^{1, k, j}+d_{\text {intra }}^{1, k, j}\right)$, where $d_{\text {intra }}^{1, k, j}$ is perpendicular to the orientation of the vector magnetic moment. The doublet strength, defined as $\mid I_{d}^{1, k, j} d_{\text {intra }}^{1, k, j}$, is taken to be equal to $M_{1, \mathrm{k}, \mathrm{j}}=\left|\mathbf{M}_{\text {intra }}^{\mathrm{l}, \mathrm{k}, \mathrm{j}}\right|$. Let $M_{\text {intra, }}^{1, \mathrm{k}, \mathrm{j}}$ and $M_{\text {intra,y }}^{1, \mathrm{k}, \mathrm{j}}$ designate the $x$ - and $y$-components of the vector magnetic moment, we thus have 


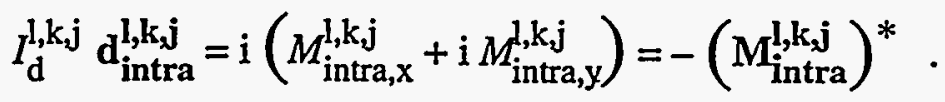

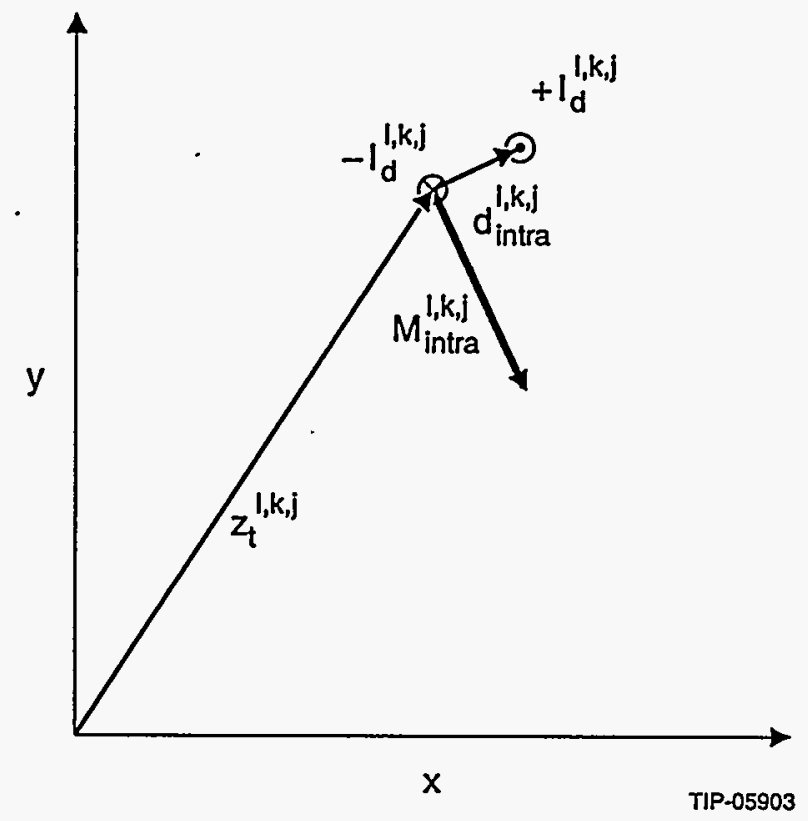

FIGURE 16: Representation of a vector magnetic moment by a current line doublet.

From the expressions derived above for the field produced by a current-line within a

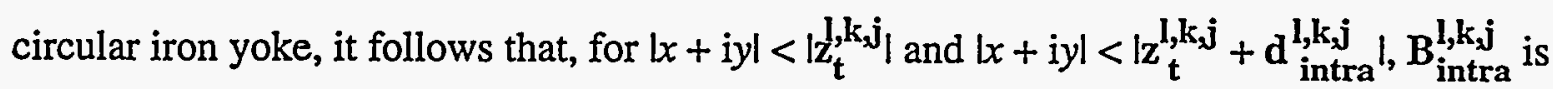
given by

$$
\mathbf{B}_{\text {intra }}^{1, k_{\mathbf{j}} \mathbf{j}}(x+\mathrm{i} y)=\sum_{n=0}^{\infty}\left(B_{\mathrm{n}, \mathrm{intra}}^{\mathrm{l}, \mathrm{k}, \mathrm{j}}+\mathrm{i} A_{\mathrm{n}, \mathrm{intra}}^{1, \mathrm{k}, \mathrm{j}}\right)\left(\frac{x+\mathrm{i} y}{R_{\text {ref }}}\right)^{\mathrm{n}}
$$

where

$$
\begin{aligned}
& B_{n, \text { intra }}^{1, k, j}+\mathrm{i} A_{n, \text { intra }}^{1, k, j}=-\frac{\mu_{0} I_{d}^{1, k, j}}{2 \pi} R_{\operatorname{ref}^{n}}\left\{-\left(z_{t}^{1, k, j}\right)^{-(n+1)}+\left(z_{t}^{1, k, j}+d_{\text {intra }}^{l, k, j}\right)^{-(n+1)}\right. \\
& \left.+\frac{\mu-1}{\mu+1} \frac{-\left[\left(z_{t}^{l, k, j}\right)^{*}\right]^{n+1}+\left[\left(z_{t}^{1, k, j}+d_{\text {intra }}^{l, k, j}\right)^{*}\right]^{n+1}}{R_{y^{2(n+1)}}}\right\} .
\end{aligned}
$$


For $\left.\right|_{\text {intra }} ^{1, k, j}|<<| z_{t}^{1, k, j} \mid$, we can write

$$
\begin{aligned}
\left(z_{t}^{1, k_{j} \mathbf{j}}+d_{\text {intra }}^{l, k_{j} \mathbf{j}}\right)^{-(n+1)} & =\left(z_{t}^{1, k, j}\right)^{-(n+1)}\left(1+\frac{d_{i n t r a}^{l, k, j}}{z_{t}^{l, k, j}}\right)^{-(n+1)} \\
& \approx\left(z_{t}^{l, k, j}\right)^{-(n+1)}-(n+1) d_{\text {intra }}^{l, k, j}\left(z_{t}^{l, k, j}\right)^{-(n+2)} .
\end{aligned}
$$

Similarly, we have

$$
\left[\left(\mathrm{z}_{\mathrm{t}}^{1, \mathbf{k}_{\mathbf{j}} \mathbf{j}}+\mathrm{d}_{\mathrm{intra}}^{\mathrm{l}, \mathrm{k}, \mathbf{j}}\right)^{*}\right]^{\mathrm{n}+1} \approx\left[\left(\mathrm{z}_{\mathrm{t}}^{\mathrm{l}, \mathrm{k}, \mathbf{j}}\right)^{*}\right]^{\mathrm{n}+1}+(n+1)\left(\mathrm{d}_{\text {intra }}^{\mathrm{l}, \mathrm{k}, \mathbf{j}}\right)^{*}\left[\left(\mathrm{z}_{\mathrm{t}}^{\mathrm{l}, \mathrm{k}, \mathbf{j}}\right)^{*}\right]^{\mathrm{n}}
$$

Combining Eqs. (33), (35), (36a), and (36b) then yields

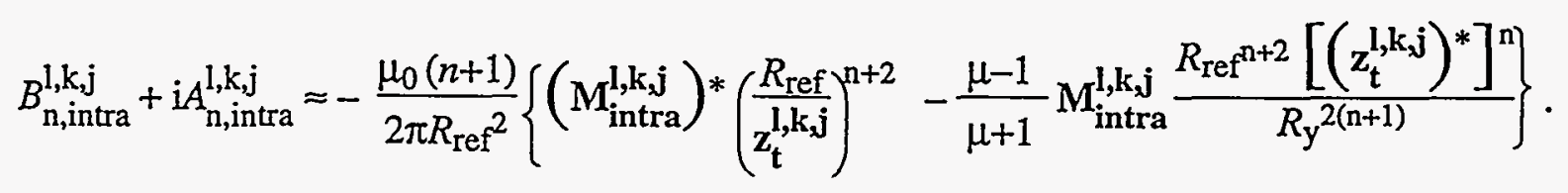

It can be seen from Eq. (37) that the dependence of $A_{\mathrm{n}, \mathrm{intra}}^{1, \mathrm{k}, \mathrm{j}}$ and $B_{\mathrm{n}, \mathrm{intra}}^{1, \mathrm{k}, \mathrm{j}}$ on the ramp rate is determined by the dependence of $\mathbf{M}_{\mathbf{i n t r a}}^{\mathbf{l}, \mathbf{k}, \mathbf{j}}$, which were shown to vary linearly as a function of $\left(\mathrm{d} I_{\mathrm{t}} / \mathrm{d} t\right)$.

The overall field, $\mathbf{B}_{\text {intra, }}$, produced by the intra-strand eddy currents generated within the magnet coil is obtained by summing the contributions of all the magnetic moments. Within the coil aperture, $B_{\text {intra }}$ may be written as

$$
\mathbf{B}_{\text {intra }}(x+\mathrm{i} y)=\sum_{n=0}^{\infty}\left(B_{\mathrm{n}, \text { intra }}+\mathrm{i} A_{\mathrm{n}, \text { intra }}\right)\left(\frac{x+\mathrm{i} y}{r_{0}}\right)^{\mathrm{n}},
$$

where $A_{\mathrm{n} \text {,intra }}$ and $B_{\mathrm{n} \text {,intra }}$ are the intra-strand eddy current components of the $(2 n+1)$-pole fields given by 


$$
B_{\mathrm{n}, \text { intra }}+\mathrm{i} A_{\mathrm{n}, \text { intra }}=\sum_{l=1}^{2} \sum_{k=1}^{K_{1}} \sum_{j=1}^{n_{1, k}} B_{n, \text { intra }}^{1, \mathrm{k}, \mathrm{j}}+\mathrm{i} A_{\mathrm{n}, \text { intra }}^{1, \mathrm{k}, \mathrm{j}}
$$

Similarly, the overall power, $P_{\text {intra }}$, dissipated per coil unit length by the intra-strand eddy currents is given by

$$
P_{\text {intra }}=\sum_{l=1}^{2} \sum_{k=1}^{K_{1}} \sum_{j=1}^{n_{1, k}} P_{\text {intra }}^{1, k, j},
$$

where $P_{\text {intra }}^{1, k, j}$ is the power loss per unit length associated with $M_{\text {intra }}^{1, k, j, j}$

$$
P_{\text {intra }}^{1, k, j}=\frac{N_{1}}{n_{1, k}} \frac{2 \pi\left(R_{3}^{l}\right)^{2}}{\mu_{0}}\left|\frac{\mathrm{dB}_{\mathfrak{t}}\left(\mathrm{z}_{\mathrm{t}}^{1, k, j}\right)}{\mathrm{dt}}\right|^{2} \tau_{\mathrm{s}}^{1, \mathrm{k}, \mathrm{j}} .
$$

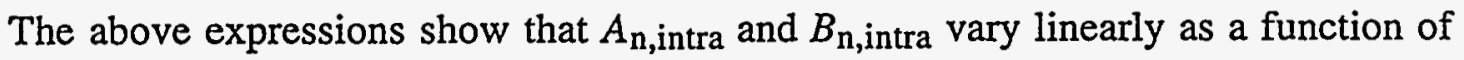
(dII/dt), while $P_{\text {intra }}$ is proportional to $\left(\mathrm{d} I_{t} / \mathrm{d} t\right)^{2}$.

7.3.3 Discussion The model described in the previous section enables one to calculate the field distortions and the power dissipation due to intra-stand eddy currents. Let us first discuss the field distortions.

As for the persistent magnetization currents, the distribution of magnetic moments determined above follows the symmetries of the transport-current field. Hence, if the strand properties are uniform, only the allowed multipole fields are affected. For a given magnetic design and given strands geometries, and assuming that the RRR is the same for the two inner and two outer coils, the dependence of $B_{2 \mathrm{~m} \text {,intra }}$ on $\left(\mathrm{d} \mathrm{I}_{\mathrm{t}} / \mathrm{d} t\right)$ can be expressed as

$$
B_{2 \mathrm{~m}, \text { intra }}=B_{2 \mathrm{~m}, \text { intra }}^{0}\left(\mathrm{RRR}, \mathrm{I}_{\mathrm{t}}\right) \frac{\mathrm{d} I_{\mathrm{t}}}{\mathrm{d} t} .
$$


Here, the dependence of $B_{2 \mathrm{~m} \text {,intra }}^{0}$ on $I_{\mathrm{t}}$ comes from the fact that, in Eq. (31), $\mathbf{M}_{\mathbf{i n t r a}}^{\mathrm{l}, \mathrm{k}_{\mathbf{j}} \mathbf{j}}$ is a function of $\tau_{s}^{1, k, j}$, which itself is a function of $\rho_{\mathrm{b}}$, and, thus, depends on the transport-current field at $\mathbf{z}_{\mathbf{t}}^{\mathbf{l}, \mathbf{k} \mathbf{j}}$.

Table VI summarizes the values of $B_{0 \text {,intra }}^{0}, B_{2 \text {,intra }}^{0}$, and $B_{4 \text {,intra }}^{0}$ obtained for the 5-cm aperture SSC dipole magnet design at 2000 A. In these computations, we assumed: $r_{\text {cus }}=$ 1.5 and $x_{\text {core }}=0.1$ for the inner-layer cable, and $r_{\text {cus }}=1.8$ and $x_{\text {core }}=0.1$ for the outerlayer cable. All the other parameters were taken at their design values. The various lines of Tab. VI correspond to various RRR values. In addition, Tab. VI lists the effective time constants of the shielding currents calculated in average over the inner-layer and outerlayer line-currents. Intra-strand eddy currents appear to have a relatively small effect on the multipole fields (for instance, the values of $B_{2 \text {,intra }}^{0}$, and $B_{4 \text {,intra }}^{0}$ are one order of magnitude smaller than the values of $S_{\mathrm{B}_{2}}$ and $S_{\mathrm{B}_{4}}$ given in Tab. IV), and, therefore, cannot account for the observed anomalous field behavior of the type-A magnets. (Note also that the signs of $B_{2 \text {,intra }}^{0}$, and $B_{4 \text {,intra }}^{0}$ are opposite to the signs of $S_{\mathrm{B}_{2}}$ and $S_{\mathrm{B}_{4}}$ ).

Let us now discuss the power dissipation. Similarly to the multipole fields, the dependence of $P_{\text {intra }}$ on $\left(\mathrm{d} I_{\mathrm{t}} / \mathrm{d} t\right)$ can be expressed as

$$
P_{\text {intra }}=P_{\text {intra }}^{0}\left(\mathrm{RRR}, I_{\mathrm{t}}\right)\left(\frac{\mathrm{d} I_{\mathrm{t}}}{\mathrm{d} t}\right)^{2}
$$

Figure 17 presents plots of $P_{\text {intra }}^{0}$ as a function of $I_{\mathrm{t}}$ for various RRR values. The power dissipation appears to decrease as a function of transport current as can be expected from the effects of magneto-resistance. 
TABLE VI: Predicted field distorsions due to intra-strand eddy currents for 5-cm aperture SSC dipole magnets at $2000 \mathrm{~A}$.

\begin{tabular}{|c|c|c|c|c|c|}
\hline \multirow[t]{2}{*}{ RRR } & \multicolumn{2}{|c|}{$t_{\mathrm{s}}$} & \multirow{2}{*}{$\begin{array}{c}B_{0, \text { intra }}^{0} \\
\text { (Gauss/(A/s)) }\end{array}$} & \multirow{2}{*}{$\begin{array}{c}B_{2, \text { intra }}^{0} \\
((\text { Gauss } /(\mathrm{A} / \mathrm{s}))\end{array}$} & \multirow{2}{*}{$\begin{array}{c}B_{4, \text { intra }}^{0} \\
\text { (Gauss/(A/s)) }\end{array}$} \\
\hline & $\begin{array}{l}\text { inner }^{\mathrm{a}} \\
\text { (ms) }\end{array}$ & $\begin{array}{l}\text { outer }{ }^{b} \\
\text { (ms) }\end{array}$ & & & \\
\hline 100 & 4.1 & 4.9 & $-3.210^{-3}$ & $-1.010^{-3}$ & $0.08210^{-3}$ \\
\hline 150 & 5.0 & 6.6 & $-3.910^{-3}$ & $-1.210^{-3}$ & $0.09810^{-3}$ \\
\hline 200 & 5.7 & 8.0 & $-4.510^{-3}$ & $-1.410^{-3}$ & $0.1110^{-3}$ \\
\hline
\end{tabular}

a In average over inner-layer line-currents.

$\mathrm{b}$ In average over outer-layer line-currents.

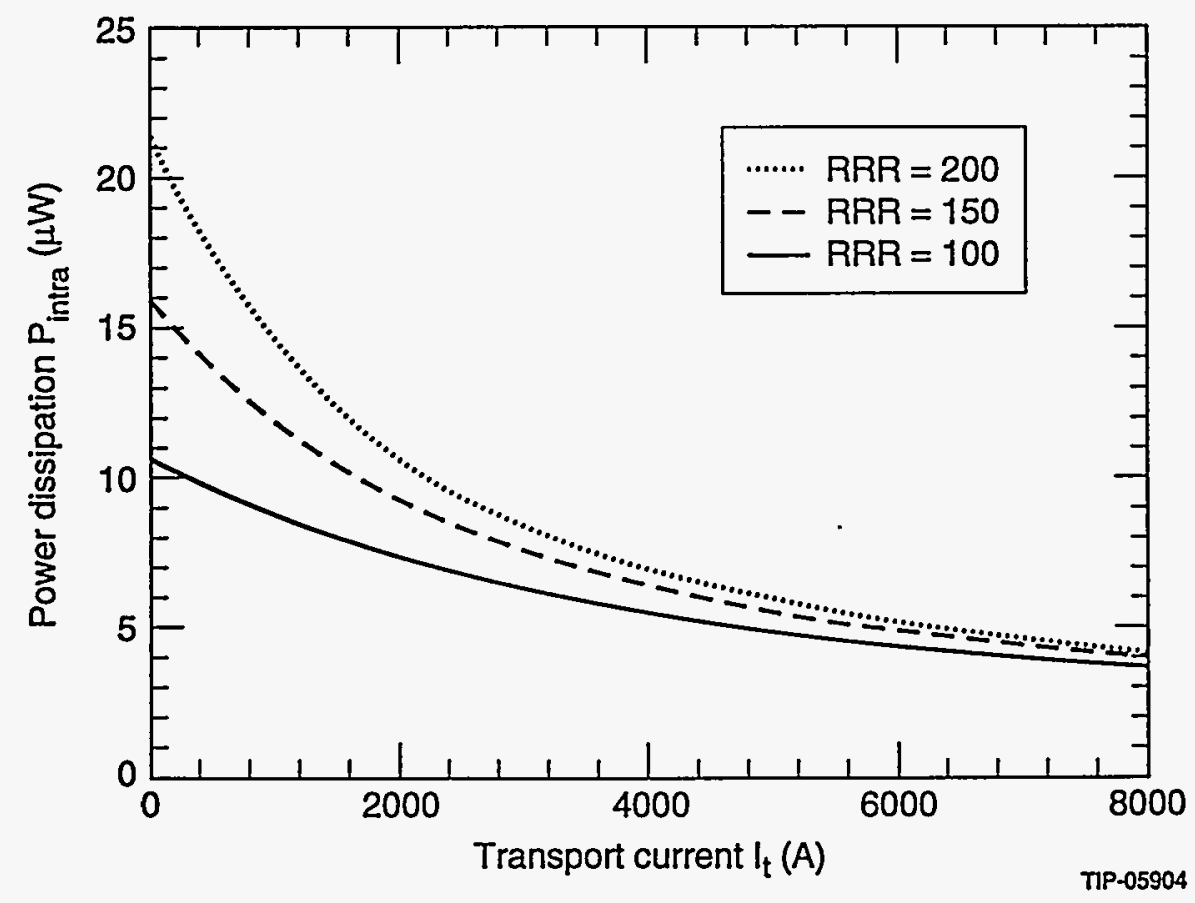

FIGURE 17: Computed power dissipated by intra-strand eddy currents per unit length of 5-cm aperture SSC dipole magnets as a function of transport current and coil RRR. (The two inner and two outer coils are assumed to have the same RRR.) 
The energy, $E_{\text {intra, }}$, dissipated per coil unit length during a current cycle can be estimated as

$$
E_{\text {intra }}=\int_{\text {cycle }} P_{\text {intra }} \mathrm{d} t=E_{\text {intra }}^{0}(\text { RRR,cycle }) \frac{\mathrm{d} I_{\mathrm{t}}}{\mathrm{d} t},
$$

where

$$
E_{\text {intra }}^{0}(R R R, \text { cycle })=\int_{\text {cycle }} P_{\text {intra }}^{0}\left(\mathrm{RRR}, I_{t}\right)\left|\mathrm{d} I_{t}\right| .
$$

For a 500-5000-500 A cycle, we get

$$
\begin{aligned}
& E_{\text {intra }}^{0}(R R R=100)=61.110^{-3} \mathrm{~J} / \mathrm{m} /(\mathrm{A} / \mathrm{s}), \\
& E_{\text {intra }}^{0}(R R R=150)=76.410^{-3} \mathrm{~J} / \mathrm{m} /(\mathrm{A} / \mathrm{s}),
\end{aligned}
$$

and

$$
E_{\text {intra }}^{0}(R R R=200)=87.510^{-3} \mathrm{~J} / \mathrm{m} /(\mathrm{A} / \mathrm{s})
$$

For a $15-\mathrm{m}$ long magnet, these values scale to numbers in the range 0.9 to $1.3 \mathrm{~J} /(\mathrm{A} / \mathrm{s})$, which appear to be of the same order of magnitude as the energy loss slopes measured on magnets DCA211, DCA318, and DCA317 at BNL (see Tab. V). Given that the time constants we calculated were smaller than those derived from test data on strand short samples, the power, and thus, the energy, we compute may be somewhat underestimated. Hence, we conclude that intra-strand eddy current losses may account for a significant fraction of the losses measured on these three magnets, while they cannot account for the larger losses measured on the other magnets. 


\subsection{Model for inter-strand eddy currents}

7.4.1 Case of a single cable Let us first consider a single Rutherford-type cable, exposed to a time-dependent external field. As we described earlier, and as is shown in Fig. 18, the Rutherford-type cables used in SSC magnets consist of a few tens of strands, twisted together, and shaped into a flat, two-layer, slightly keystoned conductor. The strands themselves are straight, except at the cable edges, where they are bent in a hairpinlike manner to ramp from one layer to the other. In such geometry, there are two types of inter-strand contacts: 1) line contacts between adjacent strands, and 2) surface contacts at the crossovers between strands of the two layers.

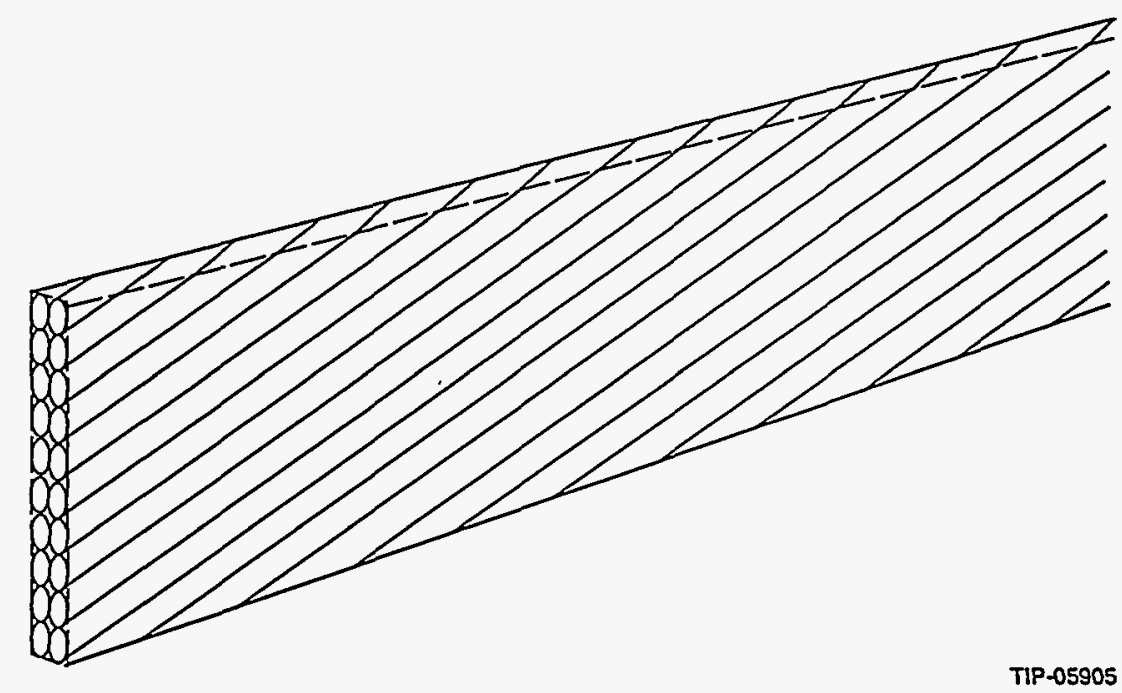

FIGURE 18: Rutherford-type cable used in superconducting particle accelerator magnets.

Following References 52 through 54, we assume that the crossover contact resistances are much smaller than the line contact resistances, and that the latter can be neglected. Hence, the smallest loops, where inter-strand eddy currents can be generated, are constituted by two adjacent strands of one layer crossing over two adjacent strands of the 
other layer. These loops are referred to as elementary loops, and the cable is represented by the model circuit of Fig. 19.

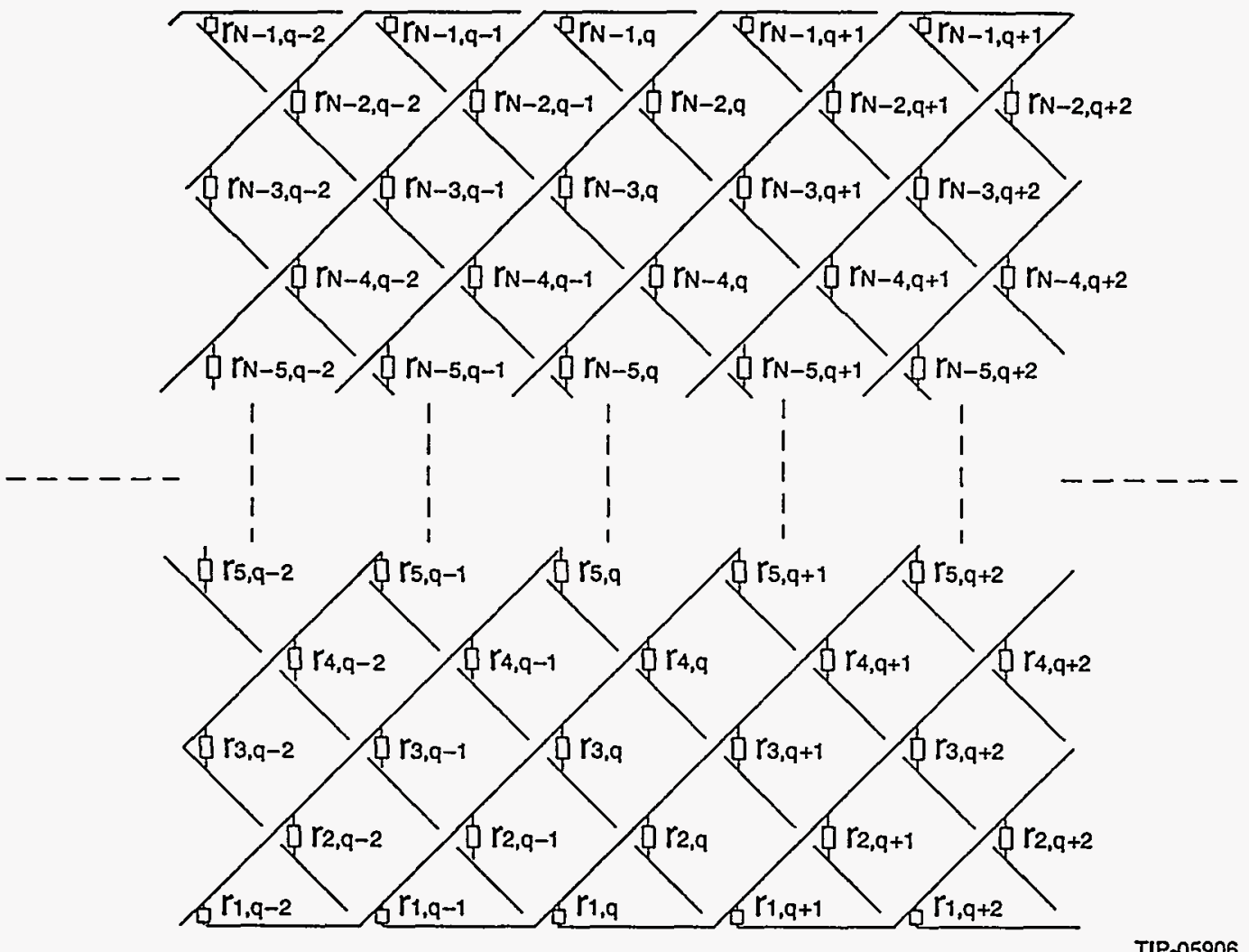

FIGURE 19: Model circuit for a $N$-strand, two-layer Rutherford-type cable exposed to a time dependent magnetic field. The cable parameters and the magnetic field, which may vary across the cable width, are assumed to be uniform along the cable length.

Two indexes are required to properly identify the crossover contacts of the model circuit of Fig. 19: one for the rows, $p$, where $1 \leq p \leq N-1$, and one for the columns, $q$, where $q$ can assume all relative integer values. A row is defined as a series of crossover contacts on a straight line parallel to the cable axis. A column is defined as a series of crossover contacts on a zigzag line across the cable width. The rows are counted starting from the thin 
edge of the slightly keystoned cable. The columns are counted from left to right, starting from an arbitrary position along the cable axis. The current circulating in a given cross-over resistance, $r_{\mathrm{p}, \mathrm{q}}$, is referred to as $i_{\mathrm{p}, \mathrm{q}}$, and is counted positively when flowing from the bottom to the top layer of the cable (see Fig. 20). The magnetic flux, $\Phi_{\mathrm{p}, \mathrm{q}}$, through an elementary loop is reckoned by the indexes of the crossover resistance at its left-hand side corner and is counted positively when penetrating the cable from the bottom. For a given column of a $N$-strand cable, there are $(N-1)$ cross-over resistances, cross-over currents, and elementary loops.
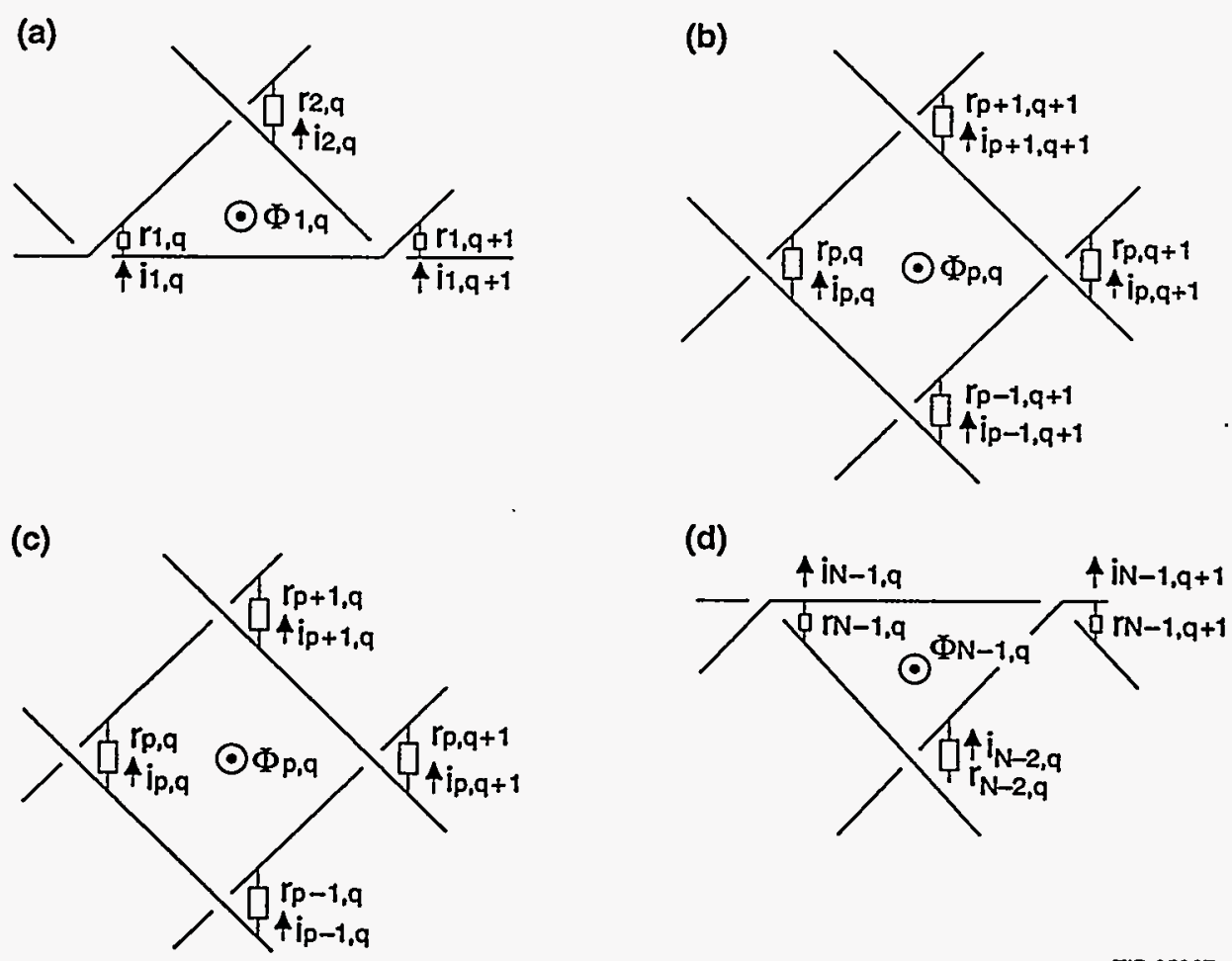

TIP-05907

FIGURE 20: Elementary loops of the model circuit for a Rutherford-type cable exposed to a time-dependent magnetic field: a) at the thin edge of the slightly keystoned cable, $b$ ) in the middle of the cable and for $p$ even, b) in the middle of the cable and for $p$ odd, and d) at the thick edge of the cable and for $N$ even. 
The crossover currents can be determined by applying Faraday's law to the $(N-1)$ elementary loops of the $q$-th column. For the loop at the cable thin edge (see Fig. 20(a)), we get

$$
r_{1, \mathrm{q}} i_{1, \mathrm{q}}+r_{1, \mathrm{q}+1} i_{1, \mathrm{q}+1}-r_{2, \mathrm{q}} i_{2, \mathrm{q}}=\frac{\mathrm{d} \Phi_{1, \mathrm{q}}}{\mathrm{d} t}
$$

For the loops in the middle of the cable, and for $p$ even, $2 \leq p \leq N-2$ (see Fig. 20(b)), we get

$$
r_{\mathrm{p}, \mathrm{q}} i_{\mathrm{p}, \mathrm{q}}+r_{\mathrm{p}, \mathrm{q}+1} i_{\mathrm{p}, \mathrm{q}+1}-r_{\mathrm{p}+1, \mathrm{q}+1} i_{\mathrm{p}+1, \mathrm{q}+1}-r_{\mathrm{p}-1, \mathrm{q}+1} i_{\mathrm{p}-1, \mathrm{q}+1}=\frac{\mathrm{d} \Phi_{\mathrm{p}, \mathrm{q}}}{\mathrm{d} t}
$$

while for $p$ odd, $3 \leq p \leq N-2$ (see Fig. 20(c)), we get

$$
r_{\mathrm{p}, \mathrm{q}} i_{\mathrm{p}, \mathrm{q}}+r_{\mathrm{p}, \mathrm{q}+1} i_{\mathrm{p}, \mathrm{q}+1}-r_{\mathrm{p}+1, \mathrm{q}} i_{\mathrm{p}+1, \mathrm{q}}-r_{\mathrm{p}-1, \mathrm{q}} i_{\mathrm{q}-1, \mathrm{q}}=\frac{\mathrm{d} \Phi_{\mathrm{p}, \mathrm{q}}}{\mathrm{d} t}
$$

For the loop at the thick edge of the cable, and if $N$ is even (see Fig. 20(d)), we get

$$
r_{\mathrm{N}-1, \mathrm{q}} i_{\mathrm{N}-1, \mathrm{q}}+r_{\mathrm{N}-1, \mathrm{q}+1} i_{\mathrm{N}-1, \mathrm{q}+1}-r_{\mathrm{N}-2, \mathrm{q}} i_{\mathrm{N}-2, \mathrm{q}}=\frac{\mathrm{d} \Phi_{\mathrm{N}-1, \mathrm{q}}}{\mathrm{d} t} \text {, }
$$

while if $N$ is odd, we get

$$
r_{\mathrm{N}-1, \mathrm{q}} i_{\mathrm{N}-1, \mathrm{q}}+r_{\mathrm{N}-1, \mathrm{q}+1} i_{\mathrm{N}-1, \mathrm{q}+1}-r_{\mathrm{N}-2, \mathrm{q}+1} i_{\mathrm{N}-2, \mathrm{q}+1}=\frac{\mathrm{d} \Phi_{\mathrm{N}-1, \mathrm{q}}}{\mathrm{d} t} .
$$

A method to determine the general solutions of the system of Eqs. (47) is developed in Reference 54 . The problem, however, can be simplified considerably by assuming that the crossover resistances, the elementary fluxes, and the crossover currents are uniform along the cable axis, and, for $p, 1 \leq p \leq N-1$, and for all $q$, satisfy the conditions 


$$
\begin{gathered}
r_{\mathrm{p}, \mathrm{q}+1}=r_{\mathrm{p}, \mathrm{q}}, \\
\Phi_{\mathrm{p}, \mathrm{q}+1}=\Phi_{\mathrm{p}, \mathrm{q}},
\end{gathered}
$$

and

$$
i_{\mathrm{p}, \mathrm{q}+1}=i_{\mathrm{p}, \mathrm{q}}
$$

Then, the dependence of Eqs. (47a) through (47e) on the column number vanishes, and the system can be written

$$
\begin{gathered}
2 r_{1} i_{1}-r_{2} i_{2}=\frac{\mathrm{d} \Phi_{1}}{\mathrm{~d} t} \\
2 r_{\mathrm{p}} i_{\mathrm{p}}-r_{\mathrm{p}+1} i_{\mathrm{p}+1}-r_{\mathrm{p}-1} i_{\mathrm{p}-1}=\frac{\mathrm{d} \Phi_{\mathrm{p}}}{\mathrm{d} t}, \quad \text { for } p, 2 \leq p \leq N-2,
\end{gathered}
$$

and

$$
2 r_{\mathrm{N}-1} i_{\mathrm{N}-1}-r_{\mathrm{N}-2} i_{\mathrm{N}-2}=\frac{\mathrm{d} \Phi_{\mathrm{N}-1}}{\mathrm{~d} t}
$$

(Note that Eqs. (49a) through (49c) apply for both $N$ even and $N$ odd.)

The solutions of the system of Eqs. (49) are

$$
i_{1}=\frac{1}{N r_{1}} \sum_{m=1}^{N-1}(N-m) \frac{\mathrm{d} \Phi_{\mathrm{m}}}{\mathrm{d} t}
$$

and

$$
i_{\mathrm{p}}=p \frac{r_{1}}{r_{\mathrm{p}}} i_{1}-\frac{1}{r_{\mathrm{p}}} \sum_{m=1}^{p-1}(p-m) \frac{\mathrm{d} \Phi_{\mathrm{m}}}{\mathrm{d} t}, \quad \quad \text { for } p, 2 \leq p \leq N-1 .
$$

In addition, the power, $P_{\mathrm{c}}$, dissipated per cable unit length by the crossover currents can be derived as 


$$
P_{\mathrm{c}}=\frac{N}{L_{\mathrm{c}}} \sum_{p=1}^{N-1} r_{\mathrm{p}} i_{\mathrm{p}}{ }^{2}
$$

where $L_{\mathrm{c}}$ is the cable pitch length.

Given the values of the elementary fluxes and of the crossover resistances, Eqs. (50a), (50b), and (51) enable one to calculate the crossover currents and their power dissipation. The next step is to calculate the eddy currents induced along the cable strands, to which we shall refer as branch currents.

Looking again at the model circuit of Fig. 19, let $J_{1, \mathrm{q}}$ designate the current circulating in the branch from $r_{1, \mathrm{q}}$ to $r_{1, \mathrm{q}+1}$ at the thin edge of the cable (see Fig. 21(a)), and let $J_{\mathrm{N}, \mathrm{q}}$ designate the current circulating in the branch from $r_{\mathrm{N}-1, \mathrm{q}}$ to $r_{\mathrm{N}-1, \mathrm{q}+1}$ at the thick edge of the cable (see Fig. 21(d)). For the top-layer branches in the middle of the cable, let $J_{\mathrm{p}, \mathrm{q}}^{\text {top }}$ designate the current circulating in the branch from $r_{\mathrm{p}-1, \mathrm{q}}$ to $r_{\mathrm{p}, \mathrm{q}}$, if $p$ is even, $2 \leq p \leq N-1$, and in the branch from $r_{\mathrm{p}-1, \mathrm{q}}$ to $r_{\mathrm{p}, \mathrm{q}+1}$, if $p$ is odd, $3 \leq p \leq N-1$ (see Figs. 21(b) and 21(c)). For the bottom-layer branches in the middle of the cable, let $J_{\mathrm{p}, \mathrm{q}}^{\mathrm{bot}}$ designates the current circulating in the branch from $r_{\mathrm{p}, \mathrm{q}}$ to $r_{\mathrm{p}-1, \mathrm{q}+1}$, if $p$ is even, $2 \leq p \leq N-1$, and in the branch from $r_{\mathrm{p}, \mathrm{q}}$ to $r_{\mathrm{p}-1, \mathrm{q}}$, if $p$ is odd, $3 \leq p \leq N-1$. For a given column of a $N$-strand cable, there are $(N-2)$ top-layer branch currents, and $(N-2)$ bottom-layer branch currents, which, with the two edge branch currents, make a total of $2(N-1)$ branch currents. 
(a)

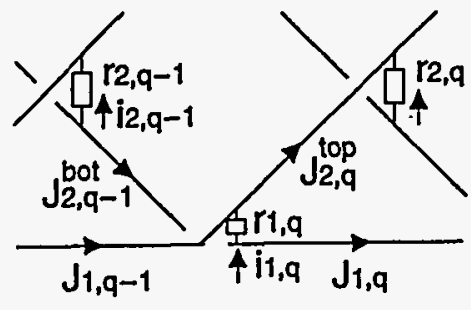

(c)

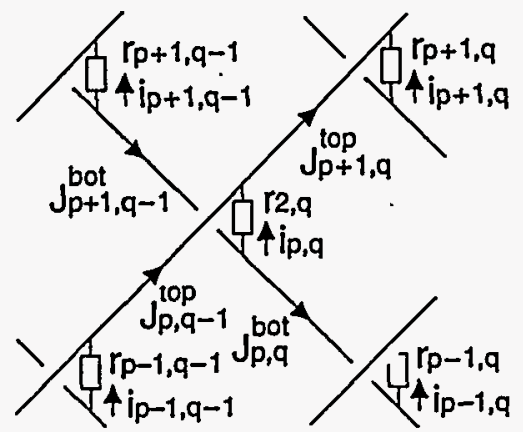

(b)

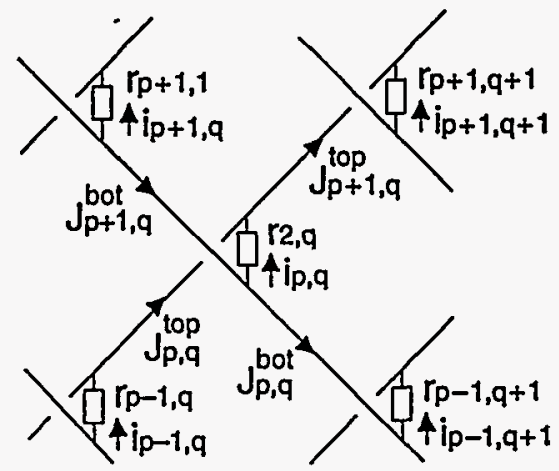

(d)

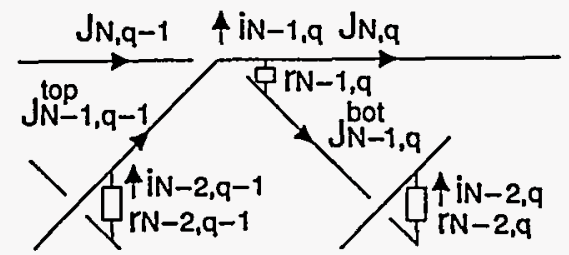

TIP-05908

FIGURE 21: Nodes of the model circuit for a Rutherford-type cable exposed to a timedependent magnetic field: a) at the thin edge of the slightly keystoned cable, b) in the middle of the cable and for $p$ even, $c$ ) in the middle of the cable and for $p$ odd, and d) at the thick edge of the cable and for $N$ even.

The branch currents can be determined by applying Kirchoff's law at the nodes at both extremities of the $(N-1)$ crossover resistances. For the top and bottom nodes of $r_{1, q}$ (see Fig. 21(a)), we get

$$
J_{1, \mathrm{q}-1}+i_{1, \mathrm{q}}=J_{2, \mathrm{q}}^{\mathrm{top}}
$$

and

$$
J_{2, \mathrm{q}-1}^{\mathrm{bot}}-i_{1, \mathrm{q}}=J_{1, \mathrm{q}}
$$


For the top and bottom nodes of $r_{\mathrm{p}, \mathrm{q}}$, and for $p$ even, $2 . \leq p \leq N-2$ (see Fig. 21(b)), we get

$$
J_{\mathrm{p}, \mathrm{q}}^{\mathrm{top}}+i_{\mathrm{p}, \mathrm{q}}=J_{\mathrm{p}+1, \mathrm{q}}^{\mathrm{top}}
$$

and

$$
J_{\mathrm{p}+1, \mathrm{q}}^{\mathrm{bot}}-i_{\mathrm{p}, \mathrm{q}}=J_{\mathrm{p}, \mathrm{q}}^{\mathrm{bot}}
$$

while for $p$ odd, $3 \leq p \leq N-2$ (see Fig. 21(c)), we get

$$
J_{\mathrm{p}, \mathrm{q}-1}^{\mathrm{top}}+i_{\mathrm{p}, \mathrm{q}}=J_{\mathrm{p}+1, \mathrm{q}}^{\mathrm{top}}
$$

and

$$
J_{\mathrm{p}+1, \mathrm{q}-1}^{\mathrm{bot}}-i_{\mathrm{p}, \mathrm{q}}=J_{\mathrm{p}, \mathrm{q}}^{\mathrm{bot}}
$$

For the top and bottom nodes of $r_{\mathrm{N}-1, \mathrm{q}}$, and if $N$ is even (see Fig. 21(d)), we get

$$
J_{\mathrm{N}-1, \mathrm{q}-1}^{\mathrm{top}}+i_{\mathrm{N}-1, \mathrm{q}}=J_{\mathrm{N}, \mathrm{q}}
$$

and

$$
J_{\mathrm{N}, \mathrm{q}-1}-\dot{i}_{\mathrm{N}-1, \mathrm{q}}=J_{\mathrm{N}-1, \mathrm{q}}^{\mathrm{bot}},
$$

while if $N$ is odd, we get

$$
J_{\mathrm{N}-1, \mathrm{q}}^{\mathrm{top}}+\dot{\mathrm{i}}_{\mathrm{N}-\mathrm{l}, \mathrm{q}}=J_{\mathrm{N}, \mathrm{q}}
$$

and

$$
J_{\mathrm{N}, \mathrm{q}-1}-\dot{i}_{\mathrm{N}-1, \mathrm{q}}=J_{\mathrm{N}-1, \mathrm{q}}^{\mathrm{bot}}
$$

The general solutions of the system of Eqs. (52) can be determined by following a method similar to the method developed in Reference 54 for the crossover currents. Once 
again, however, the problem can be simplified considerably by assuming that the crossover currents and the branch currents are uniform along the cable axis, and, for $p, 1 \leq p \leq N-1$, and for all $q$, satisfy Eq. (48c) and the conditions

$$
J_{\mathrm{p}, \mathrm{q}+1}^{\mathrm{top}}=J_{\mathrm{p}, \mathrm{q}}^{\mathrm{top}}
$$

and

$$
J_{\mathrm{p}, \mathrm{q}+1}^{\mathrm{bot}}=J_{\mathrm{p}, \mathrm{q}}^{\mathrm{bot}}
$$

With these assumptions, the dependence of Eqs. (52a) through (52j) on the column number vanishes, and it can be shown readily that

$$
J_{\mathrm{p}}^{\mathrm{bot}}=J_{\mathrm{p}}^{\mathrm{top}}, \quad \text { for } p, 2 \leq p \leq N-1 .
$$

Let $J_{\mathrm{p}}$ designate this common value. It thus appears that, in the middle of the cable, the branch currents can be regarded as flowing along zigzag paths parallel to the cable rows.

Taking Eq. (53) into account, the above system of $2(N-1)$ unknowns and $2(N-1)$ equations can be reduced to the following system of $N$ unknowns and $(N-1)$ equations

$$
J_{\mathrm{p}}=J_{\mathrm{p}-1}+i_{\mathrm{p}}, \quad \text { for } p, 2 \leq p \leq N
$$

The system of Eq. (54) has one more unknown than equation, and thus, is undetermined. An additional equation can be written by expressing that no net current is expected to result from the cable eddy currents. Hence, the sum of the branch currents flowing through a given cross-section of the conductor should be zero

$$
\sum_{p=1}^{N} J_{\mathrm{p}}=0
$$


Combining Eq. (55) and the system of Eq. (54) yields

$$
J_{1}=-\frac{1}{N} \sum_{m=1}^{N-1}(N-m) i_{\mathrm{m}}
$$

and

$$
J_{\mathrm{p}}=J_{1}+\sum_{m=1}^{p-1} i_{\mathrm{m}} . \quad \text { for } p, 2 \leq p \leq N
$$

Eqs. (56a) and (56b) enable one to calculate the branch currents as a function of the crossover currents, which, given the elementary fluxes and the crossover resistances, can be determined using Eqs. (50a) and (50b).

If we further assume that the crossover resistances and the elementary fluxes are all equal, Eqs. (50), (51), and (56) can be written

$$
\begin{array}{cc}
i_{\mathrm{p}}=\frac{p(N-p)}{2 r_{\mathrm{c}}} \frac{\mathrm{d} \Phi}{\mathrm{d} t}, & \text { for } p, 1 \leq p \leq N-1, \\
J_{\mathrm{p}}=\frac{-N\left(N^{2}-1\right)+2 p(p-1)(3 N-2 p+1)}{24 r_{\mathrm{c}}} \frac{\mathrm{d} \Phi}{\mathrm{d} t}, & \text { for } p, 1 \leq p \leq N,
\end{array}
$$

and

$$
P_{\mathrm{c}}=\frac{N^{2}\left(N^{4}-1\right)}{120 L_{\mathrm{c}} r_{\mathrm{c}}}\left(\frac{\mathrm{d} \Phi}{\mathrm{d} t}\right)^{2}
$$

where $r_{\mathrm{c}}$ and $\Phi$ designate the common values of crossover resistances and elementary fluxes. Here we have used the well known identities

$$
\sum_{p=1}^{N} p=\frac{N(N+1)}{2}
$$




$$
\sum_{p=1}^{N} p^{2}=\frac{N(N+1)(2 N+1)}{6},
$$

and

$$
\sum_{p=1}^{N} p^{3}=\frac{N^{2}(N+1)^{2}}{4}
$$

Considering that there are $N(N-1)$ elementary loops per cable pitch length, an estimate of $\Phi$ is simply given by

$$
\Phi \approx \frac{w_{\mathrm{c}} L_{\mathrm{c}}}{N(N-1)} B_{\mathrm{t}, \perp},
$$

where $w_{\mathrm{c}}$ is the cable width, and $B_{\mathrm{t}, \perp}$ is the supposedly uniform component of the external field perpendicular to the cable.

By combining Eqs. (58) and (61), on can derive the expression of the thin-edge branch current, $J_{1}$

$$
J_{1} \approx-\frac{(N+1) w_{\mathrm{c}} L_{\mathrm{c}}}{24 r_{\mathrm{c}}} \frac{\mathrm{d} B_{\mathrm{t}, \perp}}{\mathrm{d} t}
$$

which, with the thick-edge branch current, $J_{\mathrm{N}}$, can be verified to be the most intense.

By combining Eqs. (59) and (61), one can also derive the power dissipation

$$
P_{\mathrm{c}} \approx \frac{\left(N^{4}-1\right) w_{\mathrm{c}}^{2} L_{\mathrm{c}}}{120(N-1)^{2} r_{\mathrm{c}}}\left(\frac{\mathrm{d} B_{\mathrm{t}, \perp}}{\mathrm{d} t}\right)^{2} \approx \frac{N^{2} w_{\mathrm{c}}^{2} L_{\mathrm{c}}}{120 r_{\mathrm{c}}}\left(\frac{\mathrm{d} B_{\mathrm{t}, 1}}{\mathrm{~d} t}\right)^{2},
$$

where we recognize an expression similar to that presented in Reference 52. (Note that, in Eq. (63), $L_{c}$ corresponds to the full pitch length of the cable, while Reference 52 uses the cable half-pitch length). 
For the SSC inner cable, $N=30, w_{\mathrm{c}}=12.34 \mathrm{~mm}$, and $L_{\mathrm{c}}=86 \mathrm{~mm}$. Assuming a field ramp rate of $0.1 \mathrm{~T} / \mathrm{s}$ and a crossover resistance of $1 \mu \Omega$, we get: $\left|I_{1}\right| \approx 137 \mathrm{~A}$, and $P_{\mathrm{c}} \approx$ $1.1 \mathrm{~W} / \mathrm{m}$. This simple calculation shows that the branch currents can be very large and that the crossover currents can dissipate sizable power.

7.4.2 Case of a magnet coil Having treated the case of a rectilinear and infinite cable, we can now go back to the case of a magnet coil. Assuming that the elementary fluxes, the crossover resistances, the crossover currents, and the branch currents are uniform along the axis of every turn of every layer, the $\left(N_{1}-1\right)$ crossover currents, $i_{1, k, p}, 1 \leq p \leq N_{1}-1$, and $N_{1}$ branch currents, $J_{1, k, p}, 1 \leq p \leq N_{1}$, of turn $k$ of layer $l$ can be computed as

$$
i_{1, k, 1}=\frac{1}{N_{1} \eta_{1, \mathrm{k}, 1}} \sum_{m=1}^{N-1}(N-m) \frac{\mathrm{d} \Phi_{1, k, \mathrm{~m}}}{\mathrm{~d} t}
$$

and

$$
\begin{gathered}
i_{1, \mathrm{k}, \mathrm{p}}=p \frac{\eta_{1, \mathrm{k}, \mathrm{l}}}{\eta_{1, \mathrm{k}, \mathrm{p}}} i_{1, \mathrm{k}, 1}-\frac{1}{\eta_{1, \mathrm{k}, \mathrm{p}}} \sum_{m=1}^{p-1}(p-m) \frac{\mathrm{d} \Phi_{1, \mathrm{k}, \mathrm{m}}}{\mathrm{d} t}, \quad \text { for } p, 2 \leq p \leq N_{1}-1, \\
J_{1, \mathrm{k}, 1}=-\frac{1}{N_{1}} \sum_{m=1}^{N-1}(N-m) i_{1, \mathrm{k}, \mathrm{m}},
\end{gathered}
$$

and

$$
J_{1, \mathrm{k}, \mathrm{p}}=J_{1, \mathrm{k}, \mathrm{p}}+\sum_{m=1}^{p-1} i_{\mathrm{l}, \mathrm{k}, \mathrm{m}} . \quad \text { for } p, 2 \leq p \leq N
$$

where $r_{1, \mathrm{k}, \mathrm{p}}$ and $\Phi_{1, \mathrm{k}, \mathrm{p}}, 1 \leq p \leq N_{\mathrm{l}}-1$ designate the $\left(N_{1}-1\right)$ crossover resistances and elementary fluxes of the given turn. 
The position in the complex plane, $\mathrm{z}_{\Phi}^{\mathrm{l}, \mathrm{k}, \mathrm{p}}$, of the center of the $p$-th elementary loop of turn $k$ of layer $l$ can be estimated to be

$$
\begin{gathered}
\mathbf{z}_{\Phi}^{1, k, 1}=\left(1-\frac{1}{2\left(N_{l}-2\right)}\right)\left(\frac{\mathbf{Z}_{1}^{1, k}+\mathbf{Z}_{2}^{1, k}}{2}\right)+\frac{1}{2\left(N_{1}-2\right)}\left(\frac{\mathbf{Z}_{3}^{l, k}+\mathbf{Z}_{4}^{l, k}}{2}\right), \\
\mathbf{z}_{\Phi}^{1, k, p}=\left(1-\frac{p-1}{N_{l}-2}\right)\left(\frac{\mathbf{Z}_{1}^{1, k}+\mathbf{Z}_{2}^{1, k}}{2}\right)+\frac{p-1}{N_{1}-2}\left(\frac{\mathbf{Z}_{3}^{1, k}+\mathbf{Z}_{4}^{1, k}}{2}\right), \quad \text { for } p, 2 \leq p \leq N_{1}-2,
\end{gathered}
$$

and

$$
\mathbf{z}_{\Phi}^{1, \mathrm{k}, \mathrm{N}_{\mathrm{l}}-1}=\frac{1}{2\left(N_{\mathrm{l}}-2\right)}\left(\frac{\mathrm{Z}_{1}^{\mathrm{l}, \mathrm{k}}+\mathrm{Z}_{2}^{\mathrm{l}, \mathrm{k}}}{2}\right)+\left(1-\frac{1}{2\left(N_{\mathrm{l}}-2\right)}\right)\left(\frac{\mathbf{Z}_{3}^{\mathrm{l}, \mathrm{k}}+\mathbf{Z}_{4}^{\mathrm{l}, \mathrm{k}}}{2}\right)
$$

where $\mathbf{Z}_{1}^{1, k}$ through $\mathbf{Z}_{4}^{1, k}$ designate the positions of the four corners of the given turn (see Fig. 14).

Let $E_{\mathrm{x}}^{1, \mathrm{k}}$ and $E_{\mathrm{y}}^{1, \mathrm{k}}$ designate the $x$ - and $y$-components of a unit vector parallel to the midplane of turn $k$ of layer $l$. We have

$$
E_{\mathrm{x}}^{\mathrm{l}, \mathrm{k}}+\mathrm{i} E_{\mathrm{y}}^{\mathrm{l}, \mathrm{k}}=\frac{\mathrm{Z}_{3}^{1, \mathrm{k}}+\mathrm{Z}_{4}^{\mathrm{l}, \mathrm{k}}-\mathrm{Z}_{1}^{\mathrm{l}, \mathrm{k}}-\mathrm{Z}_{2}^{\mathrm{l}, \mathrm{k}}}{\left|\mathrm{Z}_{3}^{1, \mathrm{k}}+\mathrm{Z}_{4}^{1, \mathrm{k}}-\mathrm{Z}_{1}^{\mathrm{l}, \mathrm{k}}-\mathrm{Z}_{2}^{\mathrm{l}, \mathrm{k}}\right|}
$$

The components $F_{\mathrm{x}}^{\mathrm{l}, \mathrm{k}}$ and $F_{\mathrm{y}}^{\mathrm{l}, \mathrm{k}}$ of a unit vector perpendicular to the midplane of turn $k$ of layer $l$ are given by

$$
F_{\mathrm{x}}^{\mathrm{l}, \mathrm{k}}+\mathrm{i} F_{\mathrm{y}}^{\mathrm{l}, \mathrm{k}}=\mathrm{i}\left(E_{\mathrm{x}}^{\mathrm{l}, \mathrm{k}}+\mathrm{i} E_{\mathrm{y}}^{\mathrm{l}, \mathrm{k}}\right)=-E_{\mathrm{y}}^{\mathrm{l}, \mathrm{k}}+\mathrm{i} E_{\mathrm{x}}^{\mathrm{l}, \mathrm{k}}
$$

Hence, at position $\mathrm{z}_{\Phi}^{\mathbf{l}, \mathbf{k}, \mathbf{p}}$, the component of the transport-current field, $B_{\mathfrak{t}, \perp}$, perpendicular to the midplane of the cable may be derived as 


$$
\begin{aligned}
& B_{\mathrm{t}, \perp}\left(\mathrm{z}_{\Phi}^{\mathrm{l,k}, \mathrm{p}}\right)=B_{\mathrm{t}, \mathrm{x}} F_{\mathrm{x}}^{1, \mathrm{k}}+B_{\mathrm{t}, \mathrm{y}} F_{\mathrm{y}}^{1, \mathrm{k}} \\
& =\operatorname{Re}\left[\mathbf{B}_{\mathbf{t}}\left(\mathbf{z}_{\Phi}^{\mathrm{l}, \mathrm{k}, \mathrm{p}}\right)\left(E_{\mathrm{x}}^{\mathrm{l}, \mathrm{k}}+\mathrm{i} E_{\mathrm{y}}^{\mathrm{l}, \mathrm{k}}\right)\right]
\end{aligned}
$$

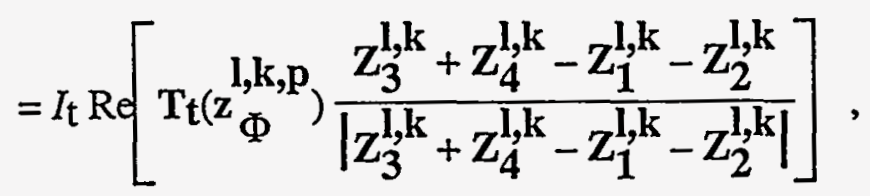

and its time derivative may derived as

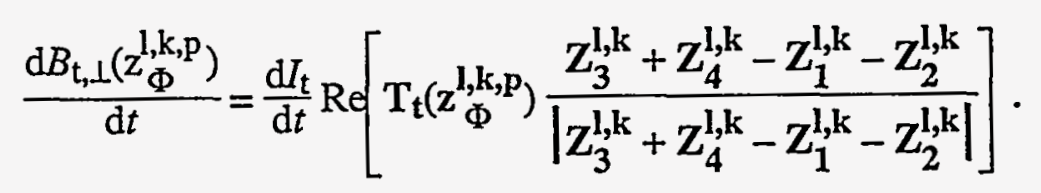

Considering that there are $N_{1}\left(N_{1}-1\right)$ elementary loops per pitch length of the layer-l cable, the time derivatives of the elementary fluxes can then be estimated as

$$
\frac{\mathrm{d} \Phi_{1, \mathrm{k}, \mathrm{p}}}{\mathrm{d} t} \approx \frac{w_{\mathrm{c}}^{1} L_{\mathrm{c}}^{1}}{2 N_{1}\left(N_{1}-1\right)} \frac{\mathrm{d} B_{\mathrm{t}, L}\left(\mathbf{z}_{\Phi}^{1, k, \mathbf{p}}\right)}{\mathrm{d} t}, \quad \text { for } p=1 \text { and } p=N_{1}-1
$$

and

$$
\frac{\mathrm{d} \Phi_{1, \mathrm{k}, \mathrm{p}}}{\mathrm{d} t} \approx \frac{w_{\mathrm{c}}^{1} L_{\mathrm{c}}^{1}}{N_{1}\left(N_{1}-1\right)} \frac{\mathrm{d} B_{\mathrm{t}, L}\left(\mathbf{z}_{\Phi}^{1, \mathbf{k}, \mathbf{p}}\right)}{\mathrm{d} t}, \quad \text { for } p, 2 \leq p \leq N_{1}-2
$$

where $w_{\mathrm{c}}^{1}$ and $L_{\mathrm{c}}^{1}$ designate the width and the pitch length of the layer-l cable. The difference between Eq. (71a) and Eq. (71b) arises from the fact that, in the model circuit of Fig. 19, the loops at the cable edges are smaller than the loops in the middle of the cable. It can be seen in Eqs. (71a) and (71b) that, as expected, the time derivatives of the elementary fluxes are proportional to $\left(\mathrm{d} I_{t} / d t\right)$. 
Given the values of the crossover resistances, and substituting the above expressions for the time derivatives of the elementary fluxes into Eqs. (64) and (65), it is now possible to calculate the crossover currents and the branch currents in each turn of the coil. As for the time derivatives of the elementary fluxes, these eddy currents are proportional to $\left(\mathrm{d} \mathrm{I}_{\mathrm{t}} / \mathrm{dt}\right)$.

In the above computation, we assumed that the crossover resistances, the elementary fluxes, and the inter-strand eddy currents were uniform along the cable axis. As a result, in the middle of the cable, the branch currents flow along zigzag paths parallel to the cable rows. Away from the cable, the field produced by a zigzag branch current can be approximated by the field produced by a rectilinear current-line of same intensity and located at the centerline of the zigzag path. Hence, the field distortions caused by the interstrand eddy currents can be calculated by associating with each turn of the coil, $N_{1}$ currentlines, of intensity, $I_{e}^{l, k, p}$, where

$$
I_{\mathrm{e}}^{1, \mathrm{k}, \mathrm{p}}=J_{1, \mathrm{k}, \mathrm{p}}, \quad \text { for } p, 1 \leq p \leq N_{\mathrm{l}},
$$

and located at $\mathbf{z}_{\mathbf{e}}^{\mathrm{l}, \mathbf{k}, \mathbf{p}}$, where

$$
z_{e}^{1, k, 1}=\frac{z_{1}^{1, k}+z_{2}^{1, k}}{2},
$$

$$
\mathbf{z}_{\mathbf{e}}^{1, k, p}=\left(1-\frac{2 p-3}{2\left(N_{1}-2\right)}\right)\left(\frac{\mathbf{Z}_{1}^{1, k}+\mathbf{Z}_{2}^{1, k}}{2}\right)+\frac{2 p-3}{2\left(N_{1}-2\right)}\left(\frac{\mathbf{Z}_{3}^{1, k}+\mathbf{Z}_{4}^{1, k}}{2}\right), \quad \text { for } p, 2 \leq p \leq N_{1}-1,
$$

and

$$
\mathrm{z}_{\mathrm{e}}^{1, \mathrm{k}, \mathrm{N}_{1}}=\frac{\mathrm{Z}_{3}^{1, \mathrm{k}}+\mathrm{Z}_{4}^{1, \mathrm{k}}}{2}
$$


The field, $\mathbf{B}_{\text {inter }}^{\mathbf{1 , k}}$, produced by the branch currents generated in turn $k$ of layer $l$ is obtained by summing the contributions from the $N_{1}$ current-lines associated with this turn, along with the contributions from the mirror images of these current lines in the iron yoke. Within the coil aperture, $\mathbf{B}_{\text {inter }}^{\mathbf{l , k}}$ may be written as

$$
\mathbf{B}_{\text {inter }}^{1, \mathrm{k}}(x+\mathrm{i} y)=\sum_{n=0}^{\infty}\left(B_{\mathrm{n}, \text { inter }}^{\mathrm{l}, \mathrm{k}}+\mathrm{i} A_{\mathrm{n}, \text { inter }}^{1, \mathrm{k}}\right)\left(\frac{x+\mathrm{i} y}{r_{0}}\right)^{\mathrm{n}},
$$

where

$$
B_{\mathrm{n}, \text { inter }}^{1, \mathrm{k}}+\mathrm{i} A_{\mathrm{n}, \text { inter }}^{1, \mathrm{k}}=-\sum_{p=1}^{N_{1}} \frac{\mu_{0} l_{\mathrm{e}}^{1, \mathrm{k}, \mathrm{p}}}{2 \pi R_{\mathrm{ref}}}\left[\left(\frac{R_{\mathrm{ref}}}{\mathrm{z}_{\mathrm{e}}^{1, \mathrm{k}, \mathrm{p}}}\right)^{\mathrm{n}+1}+\frac{\mu-1}{\mu+1}\left(\frac{R_{\mathrm{ref}}}{\mathrm{z}_{\mathrm{e}, \mathrm{m}}^{1, \mathrm{k}, \mathrm{p}}}\right)^{\mathrm{n}+1}\right] .
$$

Here, $\mathrm{z}_{\mathrm{e}, \mathrm{m}}^{\mathrm{l}, \mathrm{k}, \mathrm{p}}$ designates the location of the mirror image in the iron yoke of the current-line located at $\mathrm{z}_{\mathrm{e}}^{1, \mathrm{k}, \mathrm{p}}$ (see Eq. (9b)). It can be seen from Eq. (75) that the dependence of $A_{\mathrm{n}, \text { inter }}^{1, \mathrm{k}}$ and $B_{\mathrm{n}, \text { inter }}^{1, \mathrm{k}}$ on the ramp rate is determined by the dependence of $I_{\mathrm{e}}^{1, \mathrm{k}, \mathrm{p}}$, which were shown to vary linearly as a function of $\left(\mathrm{d} I_{t} / \mathrm{dt}\right)$.

The overall field, $\mathbf{B}_{\text {inter, }}$, produced by the inter-strand eddy currents generated within the magnet coil is obtained by summing the contributions from each turn. Within the coil aperture, we simply have

$$
\mathbf{B}_{\text {inter }}(x+\mathrm{i} y)=\sum_{n=0}^{\infty}\left(B_{\mathrm{n}, \mathrm{inter}}+\mathrm{i} A_{\mathrm{n}, \mathrm{inter}}\right)\left(\frac{x+\mathrm{i} y}{r_{0}}\right)^{\mathrm{n}}
$$

where $A_{\mathrm{n}, \text { inter }}$ and $B_{\mathrm{n} \text {,inter }}$ are the inter-strand eddy current components of the (2n+1)-pole fields given by 


$$
B_{\mathrm{n}, \text { inter }}+\mathrm{i} A_{\mathrm{n}, \text { inter }}=\sum_{l=1}^{2} \sum_{k=1}^{K_{1}} B_{\mathrm{n}, \text { inter }}^{\mathrm{l}, \mathrm{k}}+\mathrm{i} A_{\mathrm{n}, \text { inter }}^{\mathrm{l}, \mathrm{k}}
$$

Similarly, the overall power, $P_{\text {inter }}$, dissipated per coil unit length by the intra-strand eddy currents is given by

$$
P_{\text {inter }}=\sum_{l=1}^{2} \sum_{k=1}^{K_{1}} P_{\text {inter }}^{1, \mathrm{k}},
$$

where $P_{\text {inter }}^{1, k}$ is the power loss per unit length of turn $k$ of layer $l$

$$
P_{\text {inter }}^{1, \mathrm{k}}=\frac{N_{1}}{L_{\mathrm{c}}^{1}} \sum_{p=1}^{N_{\mathrm{l}}-1} \eta_{1, \mathrm{k}, \mathrm{p}} i_{\mathrm{p}}^{2}
$$

The above expressions show that $A_{\mathrm{n}, \text { inter }}$ and $B_{\mathrm{n}, \text { inter }}$ vary linearly as a function of ( $\mathrm{d}_{\mathrm{t}} / \mathrm{dt}$ ), while $P_{\text {inter }}$ is proportional to $\left(\mathrm{d}_{\mathrm{t}} / \mathrm{dt}\right)^{2}$.

7.4.3 Discussion The model described in the previous section enables one to calculate the field distortions and the power dissipation due to inter-strand eddy currents. The field distortions and the power dissipation are mainly determined by the values of crossover currents and branch currents, which themselves depend on the values of elementary fluxes and crossover resistances. Hence, for a given magnetic design and given cable geometries, the only variables in the model are the ramp rate and the values of crossover resistances. Let us, for now, assume that the crossover resistance is uniform throughout the coils, and let $r_{\mathrm{c}}$ designate this common value.

Similarly to the persistent magnetization currents and the intra-strand eddy currents, the distribution of inter-strand eddy currents determined above follows the symmetry of the 
transport-current field. Hence, if the crossover resistance is uniform, only the allowed multipole fields are affected. In this case, the dependence of $B_{2 \mathrm{~m} \text {,inter }}$ and $P_{\text {inter }}$ on $\left(\mathrm{d} I_{\mathrm{t}}, \mathrm{d} t\right)$ and $r_{\mathrm{c}}$ can be expressed as

$$
B_{2 \mathrm{~m}, \text { inter }}=\frac{B_{2 \mathrm{~m}, \text { inter }}^{0}}{r_{\mathrm{c}}} \frac{\mathrm{d} I_{\mathrm{t}}}{\mathrm{d} t}
$$

and

$$
P_{\text {inter }}=\frac{P_{\text {inter }}^{0}}{r_{\mathrm{c}}}\left(\frac{\mathrm{d} I_{\mathrm{t}}}{\mathrm{d} t}\right)^{2}
$$

In Eqs. (80) and (81), the inverse proportionality of $B_{2 \mathrm{~m} \text {,inter }}$ and $P_{\text {inter }}$ to the crossover resistance comes from the fact that the branch currents and the crossover currents are themselves inversely proportional to $r_{\mathrm{c}}$.

Table VII summarizes the values of $B_{0, \text { inter }}^{0}, B_{2, \text { inter }}^{0}, B_{4 \text {,inter }}^{0}$, and $P_{\text {inter }}^{0}$ obtained for a number of superconducting dipole magnet designs built around the world. Table VII includes the 5-cm aperture SSC dipole magnet design discussed in this paper, along with the old 4-cm aperture dipole magnet design considered at the beginning of the SSC project, 55 and an alternate $5-\mathrm{cm}$ aperture dipole magnet design (referred to as SSC/HEB), developed by SSCL, and given as a built-to-print package to WEC for their short model magnet program. ${ }^{56}$ It also includes the results of computations for the Tevatron dipole magnets, 57 the HERA dipole magnets, ${ }^{58}$ and the dipole magnets for the Relativistic Heavy 
TABLE VII: Predicted field distortions and power dissipation due to inter-strand eddy currents for various superconducting dipole magnet designs.

\begin{tabular}{|c|c|c|c|c|c|c|}
\hline & HERA & RHIC & $\begin{array}{c}\text { SSC } \\
\text { 4-cm Apert. }\end{array}$ & $\begin{array}{c}\text { SSC } \\
\text { 5-cm Apert. }\end{array}$ & SSC/HEB & Tevatron \\
\hline Transfer Function $(\mathrm{T} / \mathrm{kA})^{\mathrm{a}}$ & 0.935 & 0.709 & 1.025 & 1.048 & 1.028 & 0.953 \\
\hline $\begin{array}{l}\text { Inner Cable } \\
\text { Width (mm) } \\
\text { Twist Pitch (mm) } \\
\text { Number of Strands }\end{array}$ & $\begin{array}{l}10 \\
95 \\
24\end{array}$ & $\begin{array}{l}9.73 \\
74 \\
30\end{array}$ & $\begin{array}{c}9.296 \\
79 \\
23\end{array}$ & $\begin{array}{l}12.34 \\
86 \\
30\end{array}$ & $\begin{array}{l}12.34 \\
86 \\
30\end{array}$ & $\begin{array}{l}7.8 \\
57 \\
23\end{array}$ \\
\hline $\begin{array}{l}\text { Outer Cable } \\
\text { Width (mm) } \\
\text { Twist Pitch (mm) } \\
\text { Number of Strands }\end{array}$ & $\begin{array}{l}10 \\
95 \\
24\end{array}$ & $\begin{array}{l}\mathrm{n} / \mathrm{a} \\
\mathrm{n} / \mathrm{a} \\
\mathrm{n} / \mathrm{a}\end{array}$ & $\begin{array}{l}9.728 \\
74 \\
30\end{array}$ & $\begin{array}{l}11.68 \\
94 \\
36\end{array}$ & $\begin{array}{l}11.68 \\
94 \\
36\end{array}$ & $\begin{array}{l}7.8 \\
57 \\
23\end{array}$ \\
\hline Field Distortions ${ }^{\mathrm{b}}$ & & & & & & \\
\hline 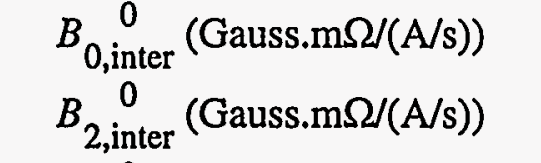 & +0.4 & +0.2 & +0.04 & +0.4 & +0.4 & 0.2 \\
\hline$B_{4, \text { inter }}^{0}($ Gauss.m $\Omega /(\mathrm{A} / \mathrm{s}))$ & -0.05 & -0.02 & -0.05 & -0.02 & -0.01 & -0.01 \\
\hline $\begin{array}{l}\text { Power Dissipation } \\
\quad P_{\text {inter }}^{0}\left(10^{-3} \mathrm{~W} \cdot \mathrm{m} \Omega / \mathrm{m} /(\mathrm{A} / \mathrm{s})^{2}\right)\end{array}$ & 0.9 & 0.3 & 0.7 & 2.4 & 2.2 & 0.4 \\
\hline
\end{tabular}

a On magnet axis.

${ }^{b}$ At the reference radius ( $1 \mathrm{~cm}$ for $\mathrm{SSC}$ designs, $2.5 \mathrm{~cm}$ for all other designs). 
Ion Collider (RHIC) now under construction at BNL. 59 The values in Tab. VII correspond to cases where all crossover contacts are assumed to be conductive. For the SSC designs, the multipole field values are quoted at a reference radius of $1 \mathrm{~cm}$, while for all other designs, the reference radius is $2.5 \mathrm{~cm}$. Table VII also lists the cable parameters relevant to the computation of inter-strand eddy currents.

In the case of the HERA dipole magnets, the cable strands were coated with a thin layer of $5 \mathrm{wt} \%$ silver-95 wt\% tin solder called stabrite. The purpose of this coating was to prevent the uncontrolled formation of a copper oxide layer on the strand surfaces, and to make the crossover resistances as uniform as possible along the cable and throughout the magnet coils. Measurements on short samples of HERA cables have shown: $r_{\mathrm{c}}=2.1 \pm$ $0.5 \mu \Omega .^{60}$ Introducing this value into Eq. (80) yields

$$
\frac{B_{2, \text { inter }}\left(R_{\mathrm{ref}}=2.5 \mathrm{~cm}\right)}{\left(\mathrm{d} I_{\mathrm{t}} / \mathrm{d} t\right)} \approx 0.2 \mathrm{Gauss} /(\mathrm{A} / \mathrm{s})
$$

This predicted ramp rate dependence of the normal sextupole field appears to be in good agreement with the results of magnetic measurements as a function of ramp rate recently carried out at DESY.61 Such an agreement gives us some confidence that the model we have developed has a sound basis.

In the case of the Tevatron dipole magnets, the coils were wound with so-called zebra cables.In a zebra cable, half of the strands are coated with stabrite, while the other half are coated with ebanol. Ebanol is a chemical which favors the development of black copper oxide on the strand surfaces. The stabrite- and ebanol-coated strands are alternated, yielding a pattern of black and silver stripes resembling a zebra. The purpose of this mixed 
coating was to reduce the number of crossover contacts which are conductive while still allowing some possibility of current redistribution among the cable strands. If we assume that the ebanol coating results in a perfect isolation, only the stabrite/stabrite crossovers let eddy currents flow, reducing the number of electrical paths to one fourth. Hence, compared to a case where all crossover contacts are assumed to be conductive, the effects of interstrand eddy currents for a zebra cable are expected to be four times smaller.

In the case of the SSC dipole magnets, the values quoted in Tab. VII show that the 5-cm aperture designs are much more sensitive to inter-strand eddy currents than the 4-cm aperture design. This increase in sensitivity can be understood when considering that the 5-cm aperture designs rely on cables that are wider and have a larger number of strands. For instance, Eq. (63) shows that the power dissipation per cable unit length is roughly proportional to the square of the number of strands and to the square of the cable width. Hence, when going from a $23-$ strand, $9.3-\mathrm{mm}$ wide cable to a $36-$ strand, $12.3-\mathrm{mm}$ wide cable, the power dissipation in the inner coils can be expected to increase by a factor of 3 . This ratio is consistent with the increase in power dissipation per coil unit length seen in Tab. VII.

As for the intra-strand eddy currents, the energy, $E_{\text {inter, }}$ dissipated per coil unit length during a current cycle can be estimated as

$$
E_{\text {inter }}=\int_{\text {cycle }} P_{\text {inter }} \mathrm{d} t=\frac{E_{\text {inter }}^{0}(\text { cycle })}{r_{\mathrm{c}}} \frac{\mathrm{d} I_{\mathrm{t}}}{\mathrm{d} t},
$$


where

$$
E_{\text {inter }}^{0}(\text { cycle })=\int_{\text {cycle }} P_{\text {inter }}^{0}\left|\mathrm{~d} I_{\mathrm{t}}\right|=\frac{P_{\text {inter }}^{0}}{r_{\mathrm{c}}} \int_{\text {cycle }}\left|\mathrm{d} I_{\mathrm{t}}\right|
$$

For the 5-cm aperture SSC design and a 500-5000-500 A cycle, we get

$$
E_{\text {inter }}^{0}=9000 P_{\text {inter }}^{0}=21.6 \mathrm{~J} \cdot \mu \Omega / \mathrm{m} /(\mathrm{A} / \mathrm{s})
$$

To complete this discussion of the model with uniform crossover resistance, Figs. 22(a) and 22(b) present three-dimensional plots of $A_{1, \text { inter }}^{1, \mathrm{k}}$ and $B_{2, \text { inter }}^{1, \mathrm{k}}(z$-axis) as a function of turn position in the $x-y$ plane. The computation was done for the 5-cm aperture SSC dipole magnet cross-section, with $r_{\mathrm{c}}=1 \mu \Omega$ and $\mathrm{d} I / \mathrm{d} t=1 \mathrm{~A} / \mathrm{s}$. It can be seen clearly that the amplitude and sign of the contributions to the multipole fields of the inter-strand eddy currents generated in a given coil turn strongly depend on the turn position. In the case of the skew quadrupole field, the upper coil turns all yield a negative contribution, while the lower coil turns all yield a positive contribution. If the crossover

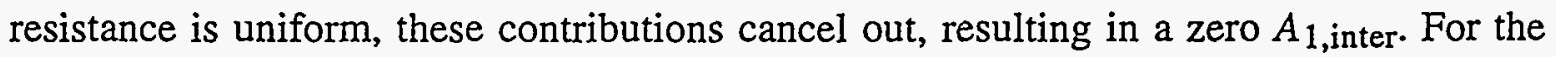
normal sextupole, the pattern is more complicated, with a change of sign in each coil quadrant, when going from the turns next to the pole to the turns close to the midplane. However, Fig. 22(b) shows also that the contributions of the turns close to the midplane are larger than the contributions of the turns next to the pole, thus resulting in a net, positive $B_{2, \text { inter. }}$ Furthermore, unlike what we obtained for the intra-strand eddy currents, the signs of $B_{2 \mathrm{~m} \text {,inter }}^{0}$ and $B_{2 \mathrm{~m} \text {,inter }}^{0}$ are the same as the signs of $S_{\mathrm{B}_{2}}$ and $S_{\mathrm{B}_{4}}$ in Tab. IV, and effects of 
(a)

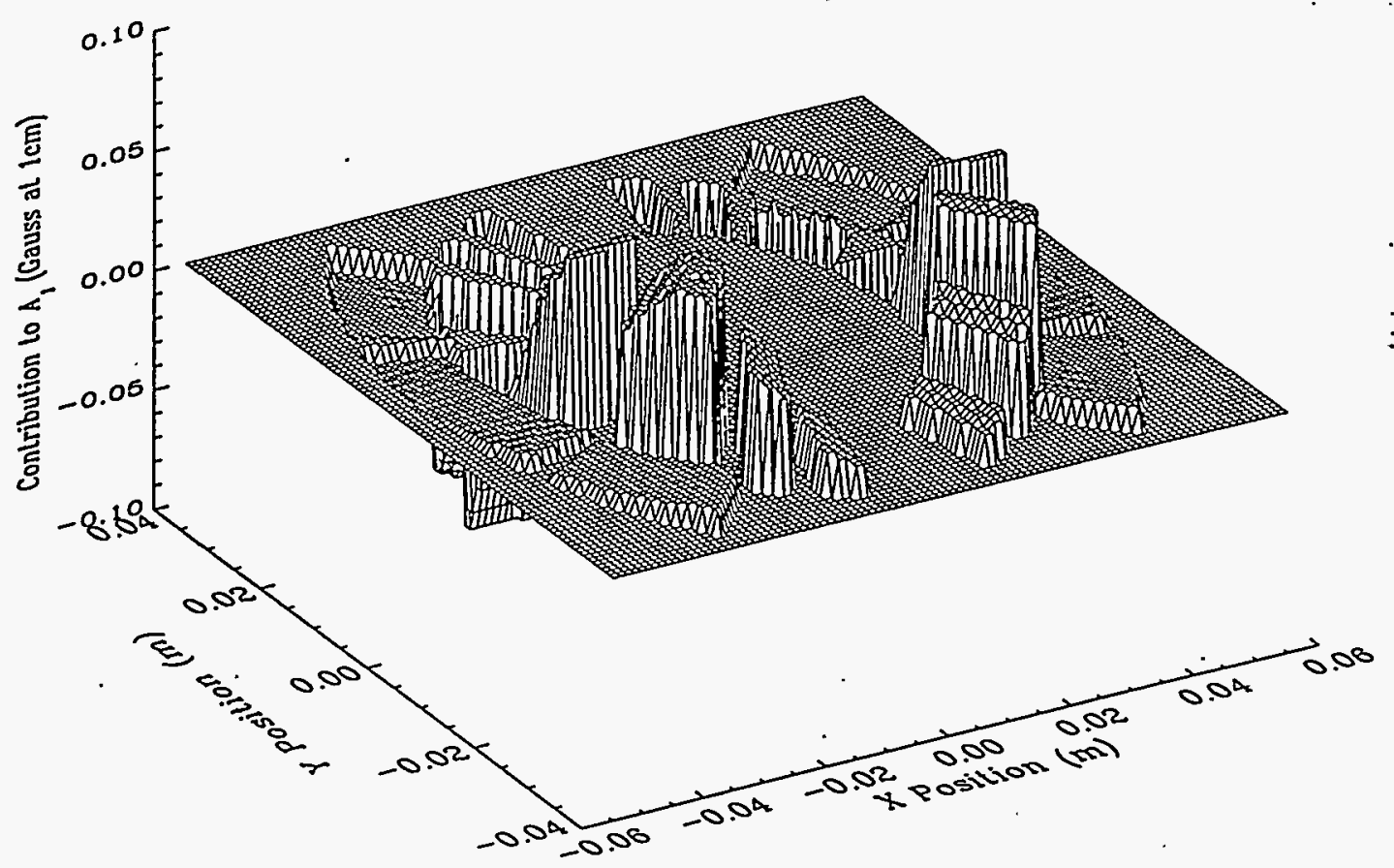

(b)

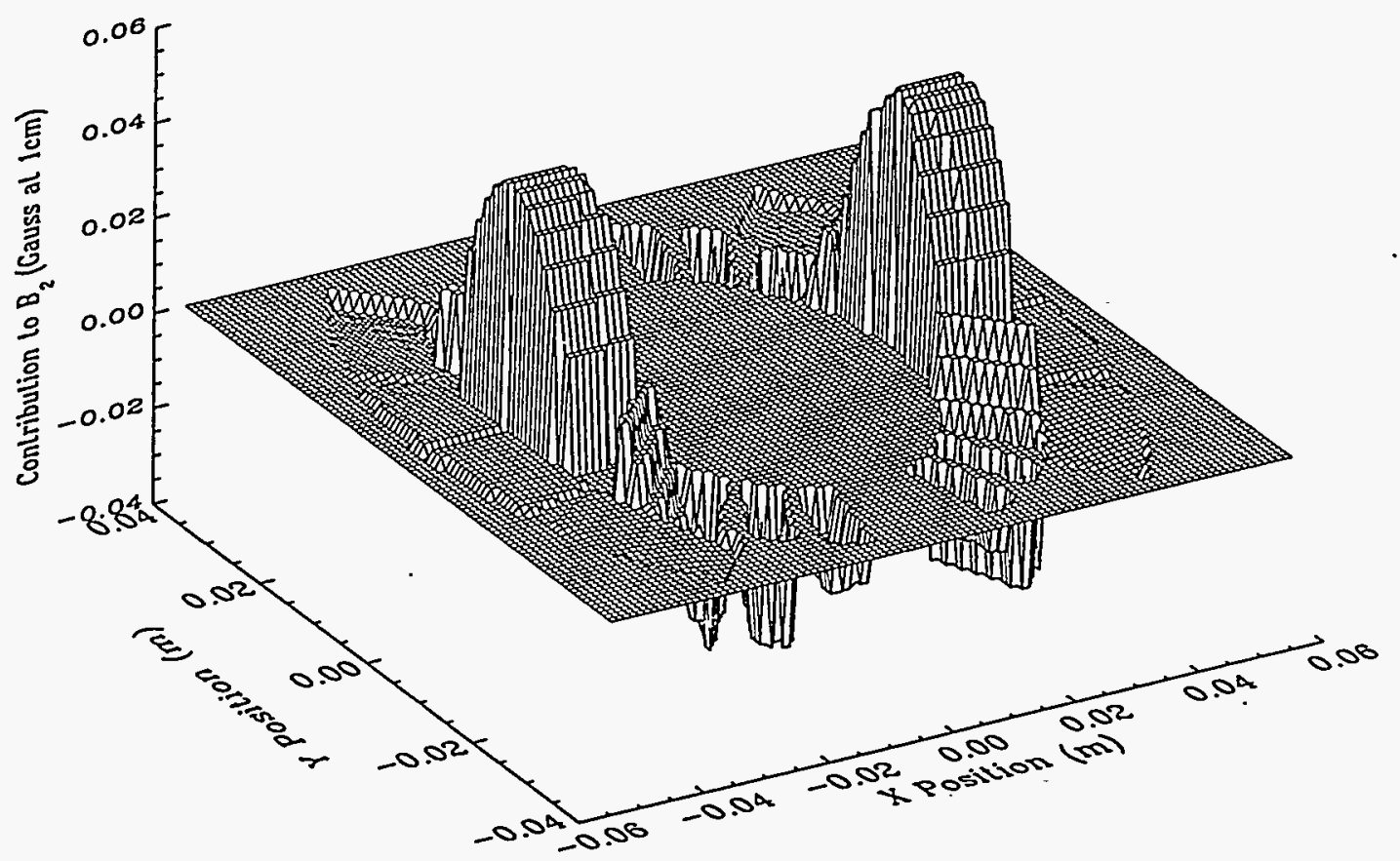

FIGURE 22: Contributions to the multipole fields of the inter-strand eddy currents generated in a given coil turn of the 5-cm aperture SSC dipole magnet cross-section as a function of turn position: a) skew quadruple field, b) normal sextupole field. The multipole fields are in Gauss. The crossover resistance is assumed to be uniform, equal to $1 \mu \Omega$, and the ramp rate is taken equal to $1 \mathrm{~A} / \mathrm{s}$. 
the same order of magnitude as $S_{\mathrm{B}_{2}}$ and $S_{\mathrm{B}_{4}}$ can be reproduced by considering crossover resistances of the order of, or less than, $10 \mu \Omega$. It thus appears that the inter-strand eddy current model we have developed may enable us to simulate behaviors similar to the observed anomalous behaviors of the type-A magnets.

\section{MODEL FOR TYPE-A BEHAVIOR}

Using the models of intra-strand and inter-strand eddy current developed above, let us now try to interpret some of the test data from the SSC dipole magnet prototypes.

\subsection{Interpretation of energy loss data}

As we described in the "Energy Loss Measurements" section (section 6), except for a number of FNAL-measured magnets where the data look suspicious, the energy loss per cycle appears to increase linearly as a function of ramp rate. This linear increase is consistent with what can be expected from the combined effects of the intra- and interstrand eddy currents. Hence, we are led to write that the slope of the measured energy losses, $S_{\mathrm{E}}$, is the sum of two terms

$$
S_{\mathrm{E}}=S_{\text {intra }}+S_{\text {inter }}
$$

where $S_{\text {intra }}$ is the slope of the intra-strand eddy current losses, and $S_{\text {inter }}$ is the slope of the inter-strand eddy current losses.

For the intra-strand losses, and assuming that the RRR is the same for the two inner and two outer coils, we simply have

$$
S_{\text {intra }}=E_{\text {intra }}^{0}(\mathrm{RRR}, \text { cycle }) L_{\mathrm{m}},
$$

where $L_{\mathrm{m}}$ designates the magnet length. 
For the inter-strand losses, and looking at Eq. (83), we are led to introduce an effective crossover resistance, $r_{c, e}$, defined as

$$
S_{\text {inter }}=\frac{E_{\text {inter }}^{0}(\text { cycle }) L_{\mathrm{m}}}{r_{\mathrm{c}, \mathrm{e}}} .
$$

This effective value corresponds to the average crossover resistance that is required to produce the observed effects.

In the case of the intra-srand eddy currents, we have shown that, for a RRR in the range 100 to 200 , and for a $500-5000-500 \mathrm{~A}$ cycle, $E_{\text {intra }}^{0}$ was in the range 0.06 to $0.09 \mathrm{~J} / \mathrm{m} /(\mathrm{A} / \mathrm{s})$. For a $15-\mathrm{m}$ long magnet, this yields values in the range 0.9 to $1.3 \mathrm{~J} /(\mathrm{A} / \mathrm{s})$. However, we also pointed out that our predictions may be somewhat underestimated. Since no systematic data on intra-strand losses are available, we thus shall use these values only for qualitative comparisons. In the case of the inter-strand eddy currents, an estimate of $E_{\text {inter }}^{0}$ is provided by Eq. (85). For a $15-\mathrm{m}$ long magnet, this yields a value of $324 \mathrm{~J} . \mu \Omega /(\mathrm{A} / \mathrm{s})$.

Looking now at the slopes of the energy loss data listed in Tab. V, it appears that, for the type-A magnets with the largest ramp rate sensitivity (magnets DCA312, DCA314, and DCA315), $S_{\mathrm{E}}$ is at least one order magnitude larger than the predicted effects of intrastrand eddy currents. Hence, it is reasonable to assume that, for these magnets, the losses are dominated by inter-strand eddy currents and that

$$
S_{\mathrm{E}} \approx S_{\text {inter }}
$$


An estimate of $r_{c, e}$ can then be obtained by combining Eqs. (88) and (89). The resulting values are listed in the last column of Tab. V. For these three magnets, the effective crossover resistance appears to be in the range 5 to $10 \mu \Omega$.

The interpretation of the energy loss data of the other magnets is somewhat more delicate. Indeed, in this case, $S_{\mathrm{E}}$ is of the same order of magnitude as the predicted effects of intra-strand eddy currents. It may even be that, for the magnets with the lowest slopes (magnets DCA211, DCA317, and DCA318 at BNL), the intra-strand eddy currents are the dominant effects. In the absence of reliable estimates for $E_{\text {intra }}^{0}$, only a lower bound of the effective crossover resistance can be obtained by writing

$$
S_{\mathrm{E}} \geq \frac{E_{\text {inter }}^{0}(\text { cycle }) L_{\mathrm{m}}}{r_{\mathrm{c}, \mathrm{e}}} .
$$

The resulting values are listed in the last column of Tab. V. (Note that the data from the FNAL-measured magnets which exhibit a quadratic increase were discarded.) It appears that the effective crossover resistances of magnets DCA317 and DCA318 are at least one order of magnitude larger than the effective crossover resistances of magnets DCA312, DCA314, and DCA315.

In summary, the ramp-rate dependence of the energy loss data of Figs. 13(a) and 13(b) can be explained in terms of intra- and inter-strand eddy currents, and the observed magnet-to-magnet variations can be explained by variations in the crossover resistances. 
8.2.1 Inter-strand eddy currents and un-allowed multipole fields As we discussed in the section "Influence of Intra-Strand Eddy Currents" (section 7.3.3), the amplitudes and signs of $B_{2 \mathrm{~m} \text {,intra }}^{0}$ and $B_{2 \mathrm{~m} \text {,intra }}^{0}$ cannot account for the values of $S_{\mathrm{B}_{2}}$ and $S_{\mathrm{B}_{4}}$ listed in Tab. IV. On the other hand, and as we discussed in the section "Influence of Inter-Strand Eddy Currents" (section 7.4.3), the signs of $B_{2 \mathrm{~m} \text {,inter }}^{0}$ and $B_{2 \mathrm{~m} \text {,inter }}^{0}$ are the same as the as the signs of $S_{\mathrm{B}_{2}}$ and $S_{\mathrm{B}_{4}}$, and effects of the same order of magnitude as $S_{\mathrm{B}_{2}}$ and $S_{\mathrm{B}_{4}}$ can be reproduced by considering crossover resistances of the order of, or less than, $10 \mu \Omega$. In addition, it appeared that the effective crossover resistances estimated from the energy loss data on magnets DCA312, DCA314, and DCA315 were in the range 5 to $10 \mu \Omega$. Interstrand eddy currents thus look like a good candidate for explaining the anomalous dynamic field behavior of the type-A magnets.

We have shown (also in section 7.4.3) that, if the crossover resistance is uniform throughout the coils, the inter-strand eddy currents only affect the allowed multipole fields. However, for the prototypes with the most anomalous dynamic field behaviors, eddycurrent-related effects were observed in most of the un-allowed multipole fields. To explain such contributions to un-allowed multipole fields, one has thus to consider eddy current distributions which violate the symmetries of the transport-current field. For instance, to explain an eddy current contribution to the skew quadrupole field, the eddy currents have to be top/bottom asymmetric. Similarly, to explain an eddy current contribution to the normal quadrupole field, the eddy currents have to be left/right asymmetric. Furthermore, in the inter-strand eddy current model the we developed, the only parameter susceptible to vary, 
and to result in asymmetric eddy current distributions, is the crossover resistance. Hence, eddy current related effects in the un-allowed multipole fields can only be explained if the crossover resistance is not uniform but is assumed to vary from turn to turn as a function of the azimuth.

8.2.2 Determining the distribution of crossover resistance Having made the assumption that the crossover resistance was not uniform, we can now, for a given magnet, try to determine the azimuthal distribution that is required to simulate the observed effects. A first guess at this distribution can be obtained by considering that the main contribution to the multipole fields comes from the eddy currents flowing near the inner radius of the inner coils. Replacing the coil by a cylindrical and non-uniform current sheet, and taking an inverse Fourier transform, one can then determine the azimuthal current distribution that is needed to generate the measured multipole fields. The peaks and valleys of this distribution indicate where the extrema of crossover resistance are located. Having guessed a profile, and relying on the slope of the energy loss data to set a scale, we can now iterate on the full model, and determine a crossover resistance distribution, which results in an eddy current distribution, that can account for both the observed field behavior and the measured energy losses.

This cumbersome iterative process can be avoided by formulating our search for a crossover resistance distribution into an optimization problem. Let us first reduce the number of free parameters by assuming that the crossover resistances of a given turn are all the same, and let $\eta_{1, \mathrm{k}}$ designates this common value. Eqs. (75) and (79) can then be written 


$$
B_{\mathrm{n}, \text { inter }}^{1, \mathrm{k}}+\mathrm{i} A_{\mathrm{n}, \text { inter }}^{1, \mathrm{k}}=\frac{D_{\mathrm{n}, \text { inter }}^{1, \mathrm{k}}}{r \mathrm{l}, \mathrm{k}} \frac{\mathrm{d} I_{\mathrm{t}}}{\mathrm{d} t}+\mathrm{i} \frac{C_{\mathrm{n}, \text { inter }}^{1, \mathrm{k}}}{r l, \mathrm{k}} \frac{\mathrm{d} I_{\mathrm{t}}}{\mathrm{d} t}
$$

and

$$
P_{\text {inter }}^{1, \mathrm{k}}=\frac{Q_{\mathrm{inter}}^{\mathrm{l}, \mathrm{k}}}{\eta, \mathrm{k}}\left(\frac{\mathrm{d} \mathrm{I}_{\mathrm{t}}}{\mathrm{d} t}\right)^{2},
$$

where $C_{\mathrm{n}, \text { inter }}^{1, \mathrm{k}} D_{\mathrm{n}, \mathrm{inter}}^{1, \mathrm{k}}$, and $Q_{\mathrm{inter}}^{1, \mathrm{k}}$ are coefficients which only depend on the geometry, and which, for a given design, can be considered as constant. In addition, by integrating Eq. (92) in a way similar to what was done for Eq. (83), the energy, $E_{\text {inter }}^{1, k}$, dissipated per unit length of turn $k$ of layer $l$ during a current cycle can be written

$$
E_{\text {inter }}^{\mathrm{l}, \mathrm{k}}=\frac{F_{\text {inter }}^{\mathrm{l}, \mathrm{k}}}{\eta_{1, \mathrm{k}}} \frac{\mathrm{d} I_{\mathrm{t}}}{\mathrm{d} t}
$$

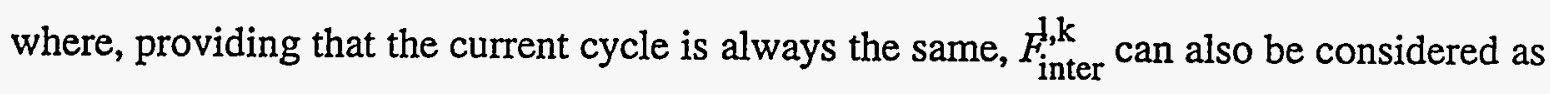
a constant coefficient.

By summing the contributions from all the turns and equating the results to the measured slopes of the multipole fields and the energy loss as a function of ramp rate, the following set of equations can be derived

$$
\begin{aligned}
& S_{\mathrm{A}_{\mathrm{n}}}=\sum_{l=1}^{2} \sum_{k=1}^{K_{1}} C_{\mathrm{n}, \text { inter }}^{\mathrm{l}, \mathrm{k}} g_{1, \mathrm{k}}, \\
& S_{\mathrm{B}_{\mathrm{n}}}=\sum_{l=1}^{2} \sum_{k=1}^{K_{1}} D_{\mathrm{n}, \text { inter }}^{\mathrm{l}, \mathrm{k}} g_{\mathrm{l}, \mathrm{k}},
\end{aligned}
$$

and

$$
\frac{S_{\mathrm{E}}}{L_{\mathrm{m}}}=\sum_{l=1}^{2} \sum_{k=1}^{K_{1}} F_{\text {inter }}^{\mathrm{l}, \mathrm{k}} g_{1, \mathrm{k}}
$$


where $g_{1, \mathrm{k}}=1 / r_{\mathrm{l}, \mathrm{k}}$ designate the crossover conductance. (In practice, equality constraints such as Eqs. (94a) and (94b) are only written for a limited number of multipole fields.)

Of course, the system of Eqs. (94) has many more unknowns than equations, and is undetermined. To converge towards a solution we therefore need to add more constraints and, if possible, an optimization function. First, we can express that the crossover conductances should all be positive. This yields the following set of inequality constraints

$$
g_{1, \mathrm{k}} \geq 0, \quad \text { for } l=1 \text { or } 2 \text {, and for } k, 1 \leq k \leq K_{1} \text {. }
$$

Second, we can express that, although the crossover conductance may vary from turn to turn, the variations are expected to be relatively smooth. Hence, we can look for the solutions of the system of Eqs. (94) which minimize the differences in conductance between adjacent turns of the same coil, as well as the overall standard deviation of the conductances over the coil cross section. This can be done by minimizing the function, $H$, defined as

$$
\begin{aligned}
H\left(g_{1, \mathrm{k}}, l=1 \text { or } 2,1 \leq k \leq K_{\mathrm{l}}\right) & =\sum_{l=1}^{2} \sum_{k=1}^{K_{1}-1} \Gamma_{\mathrm{l}, \mathrm{k}}\left(g_{1, \mathrm{k}+1}-g_{1, \mathrm{k}}\right)^{2} \\
& +\frac{\alpha}{K_{1}+K_{2}-1} \sum_{l=1}^{2} \sum_{k=1}^{K_{1}}\left(g_{1, \mathrm{k}}-\frac{1}{K_{1}+K_{2}} \sum_{q=1}^{2} \sum_{p=1}^{K_{1}} g_{\mathrm{q}, \mathrm{p}}\right)^{2} .
\end{aligned}
$$

Here, $\Gamma_{1, \mathrm{k}}=1$ if turn $k$ and $(k+1)$ are in the same coil of layer $l$, and $\Gamma_{1, \mathrm{k}}=0$ otherwise, while $\alpha$, is a free parameter which can be adjusted to get a stable solution. (In most practical cases, $\alpha$ can be set to 1 .) 
Our problem can now be formulated as determining the $\left(K_{1}+K_{2}\right)$ variables, $g_{1,1}, \ldots, g_{1, \mathrm{~K}_{1}}, g_{2,1}, \ldots, g_{1, \mathrm{~K}_{2}}$, which minimize the function $H$ defined by Eq. (96), while satisfying the equality constraints (94a), (94b), and (94c), and the inequality constraints (95). Such a problem falls into the realm of quadratic programming, ${ }^{62}$ and can be solved numerically, using for instance the subroutine $Q P R O G$ of the IMSL ${ }^{\circledR}$ MATH/LIBRARY ${ }^{\mathrm{TM}} .63$

8.2.3 Simulation results For the 5-cm aperture SSC dipole magnet design, the total number of variables (which is equal to the total number of turns) is 180 . As shown by the system of Eqs. (94), the total number of equality constraints depend on how many $S_{\mathrm{A}_{n}}$ and $S_{\mathrm{B}_{\mathrm{n}}}$ one wants to match. The computations presented below were done by matching the slopes of the multipole fields from the quadrupole $(n=1)$ to the 14-pole $(n=6)$. Hence, the total number of equality constraints was 13 , while the total number of inequality constraints was 180 . Furthermore, it was observed that, as a result of the optimization process, the crossover resistance variations in the outer coils were always small compared to the crossover resistance variations in the inner coils. Hence, the number of variables was reduced to 77 by assuming that the crossover resistance was uniform in the outer coils.

Figures 23(a) through 23(d) display the results of the optimization process described above for the data corresponding to position 1 of magnet DCA312. Figure 23(a) presents a three-dimensional plot of $g_{1, k}$ (z-axis) as a function of turn position in the $x-y$ plane. Figures 23(b) and 23(c) present similar three-dimensional plots for the resulting crossover current and branch current distributions at a ramp rate of $1 \mathrm{~A} / \mathrm{s}$, while Fig. 23(d) shows a plot of $P_{\text {inter }}^{\mathrm{l}, \mathrm{k}}$ as a function of turn position for the same ramp rate. As expected, some turns 
of the inner coils appear to have significantly larger crossover conductances than average, and these turns also correspond to peaks of eddy currents and power dissipation. The results presented here are representative of the results of the optimization process for the three axial positions on magnet DCA312, as well as of what is obtained for the other anomalous type-A magnets where measurements of the ramp rate dependence of the multipole fields are available. 


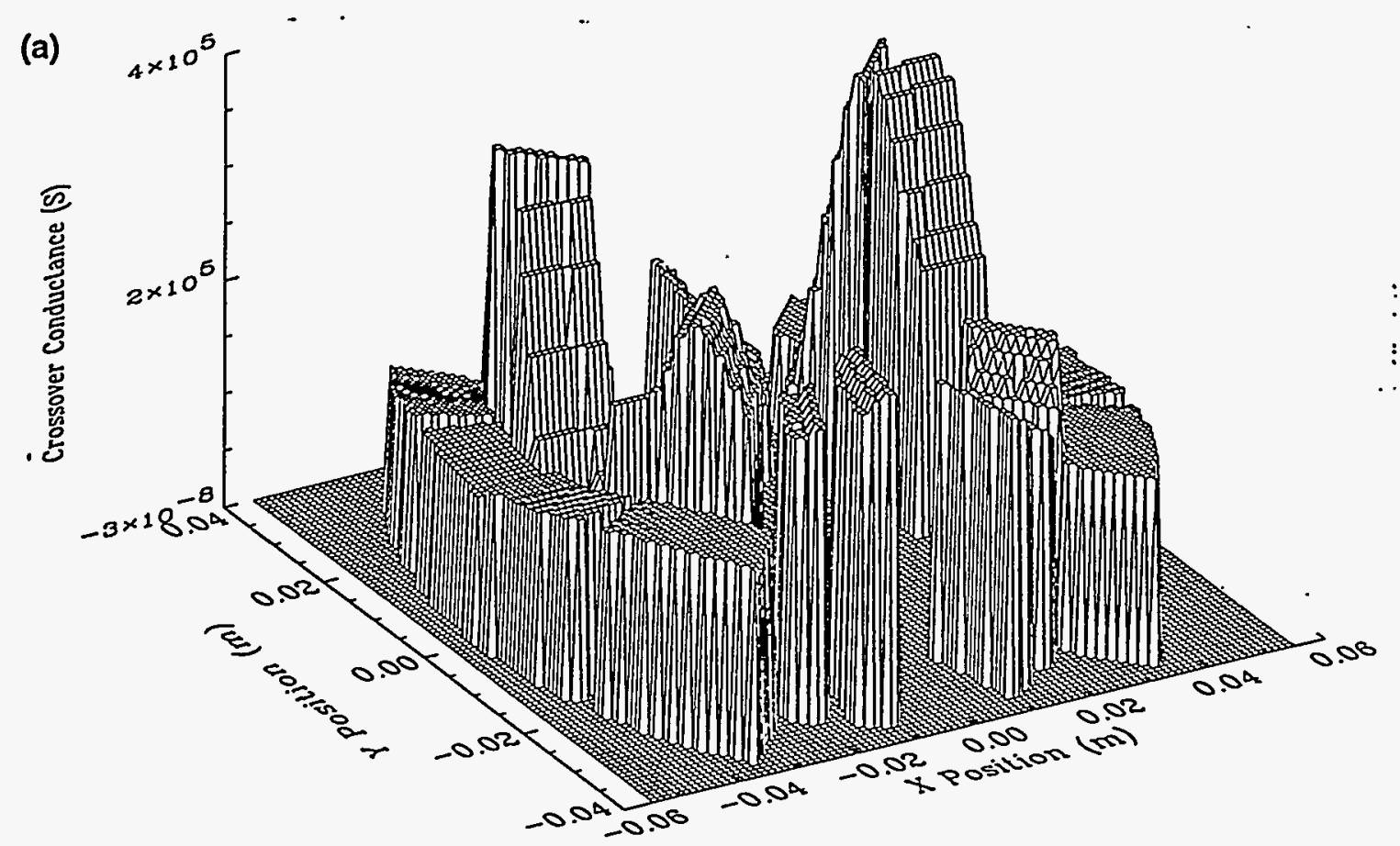

(b)

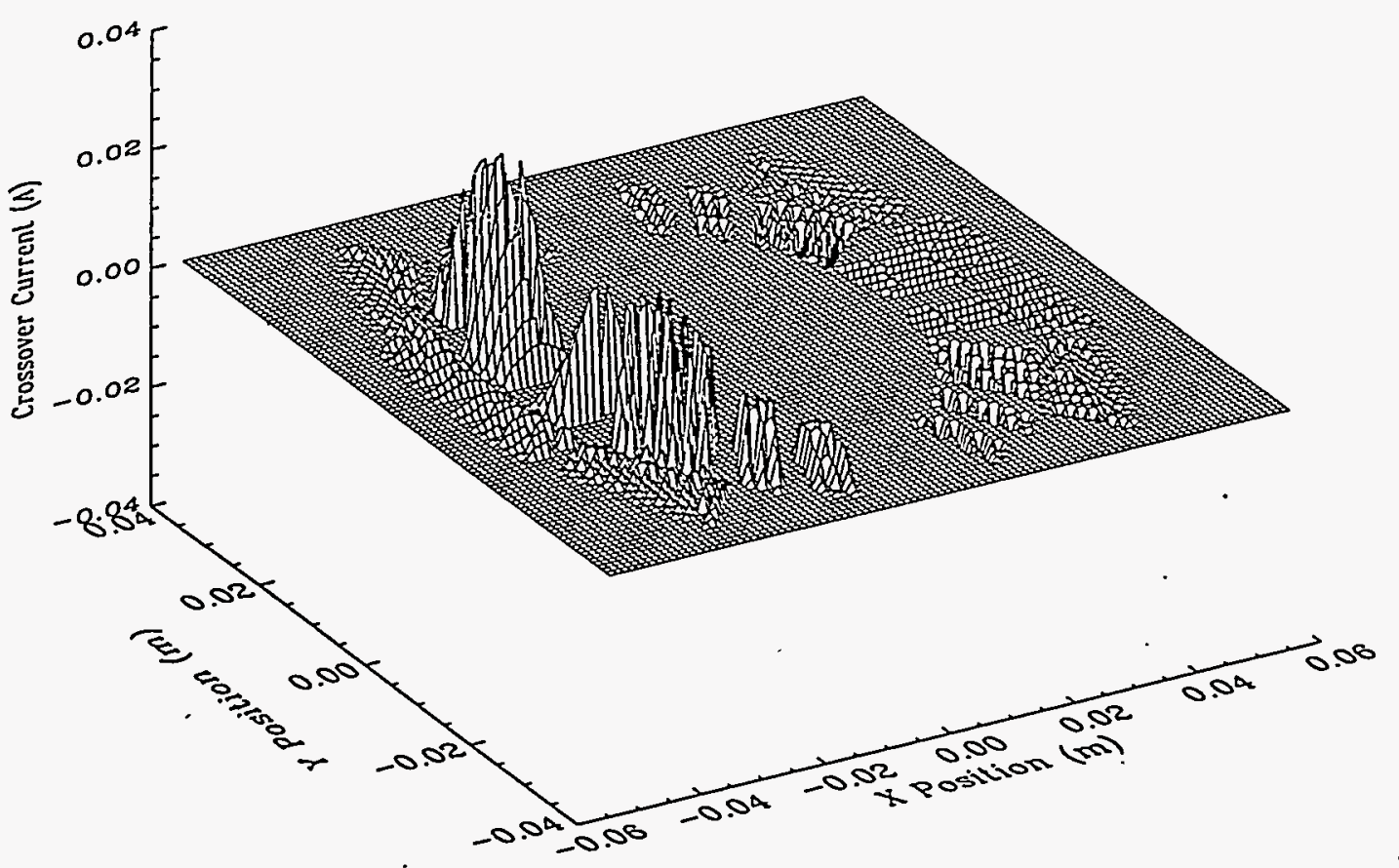

FIGURE 23: Results of the optimization process for position 1 of 5-cm aperture SSC dipole magnet prototype DCA312 at BNL: a) crossover conductance as a function of turn position, b) crossover current distribution, c) branch current distribution, and d) power dissipation per unit length as function of turn position. The ramps rate is taken equal to $1 \mathrm{~A} / \mathrm{s}$. 
(c)

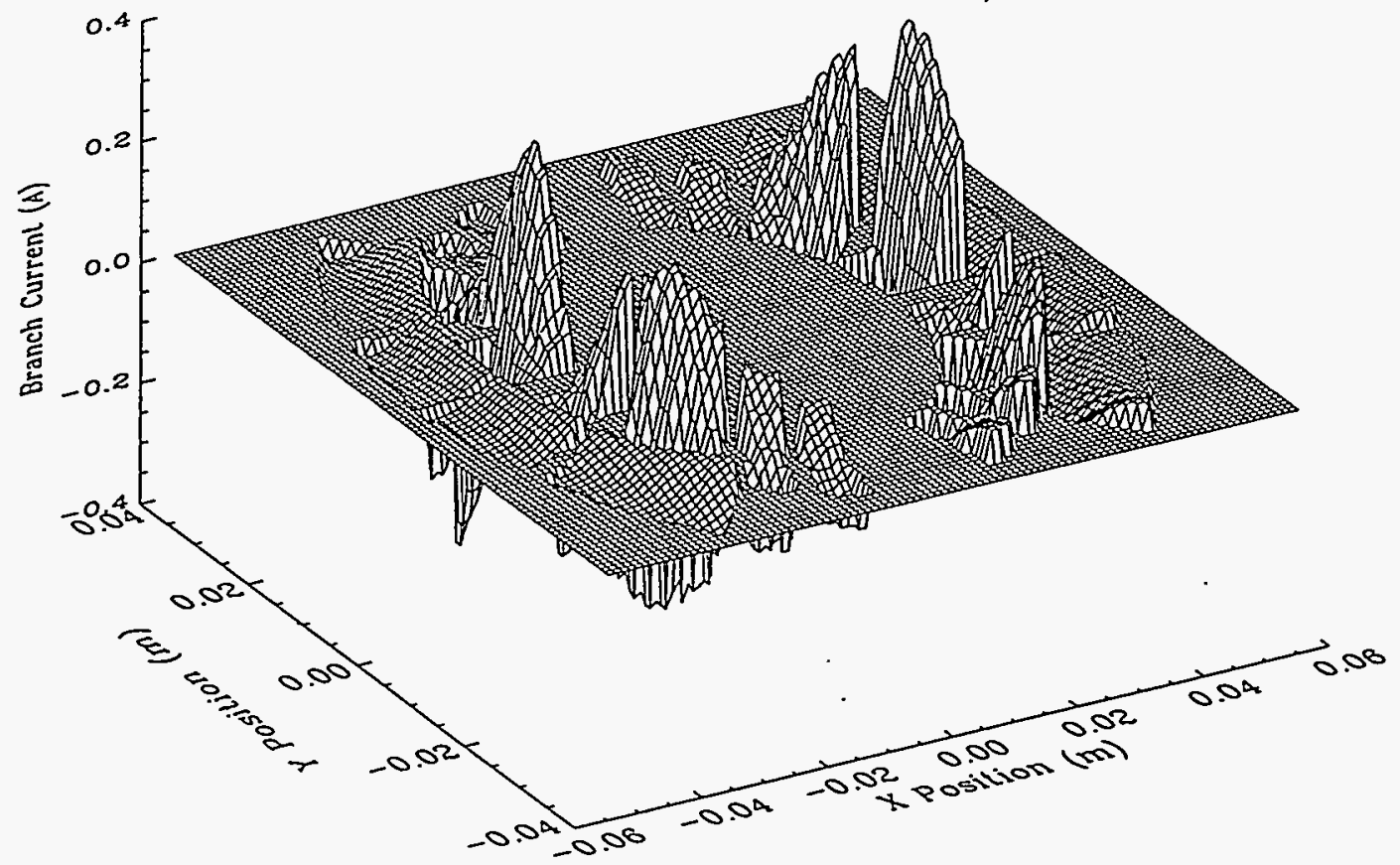

(d)

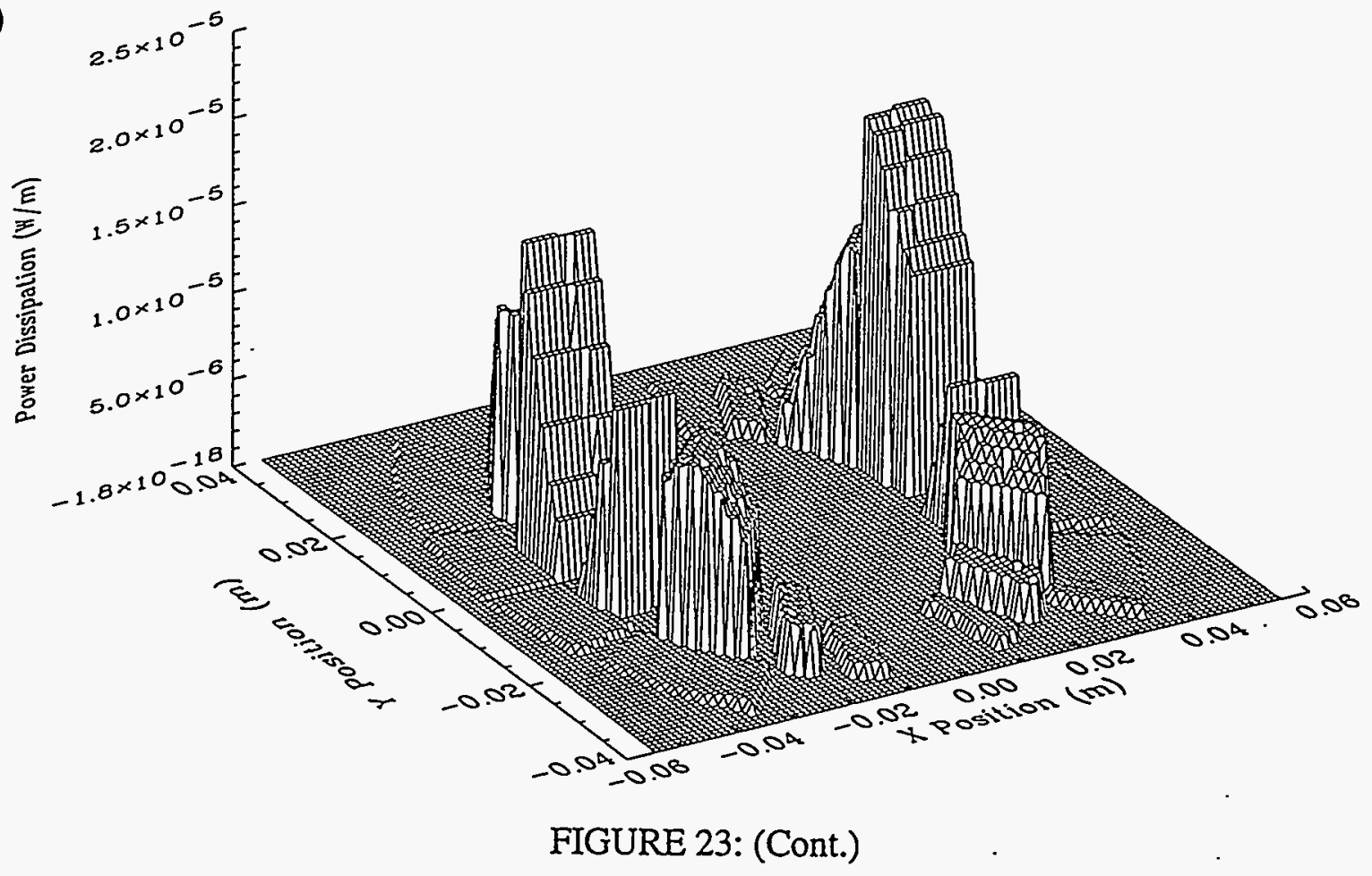

$-106$ 
8.2.4 Model validation A number of checks can be made to assess the soundness of our simulations. The most obvious one is to verify that the distributions of branch currents that we have inferred does reproduce the observed multipole field distortions. Figures 24(a) through 24(f) present comparative plots of predicted and measured multipoles fields as a function of current at position 1 of magnet DCA312 and for a ramp rate of $32 \mathrm{~A} / \mathrm{s}$. In these figures, the measured values correspond to the re-scaled multipole fields at the given position, while the computed values correspond to the sums of the predicted effects of the persistent magnetization currents and of the intra-strand and inter-strand eddy currents. It appears that, in the current range where the iron yoke saturation effects are negligible, the sum of the predicted effects is in good agreement with the measurements. This, of course, is not a surprise and only shows that our simulation process is self-consistent.

A more meaningful check can be made by comparing the azimuthal localizations of the minima of crossover resistance with the azimuthal localizations of the high ramp rate quenches. Indeed, as we already pointed out, one of the results of the optimization process is that some of the turns have a significantly lower crossover resistance than average, and that these turns also correspond to peaks of eddy currents and power dissipation. Two reasons then concur to lower the margin of these turns faster than average while ramping the magnet: 1) the overheating due to the peak of power dissipation, and 2) the larger branch currents, which, at the inner edge of the inner coil turns, flow in the same direction as the transport current. Hence, one would expect the high ramp rate quenches to originate at these locations. 
(a)

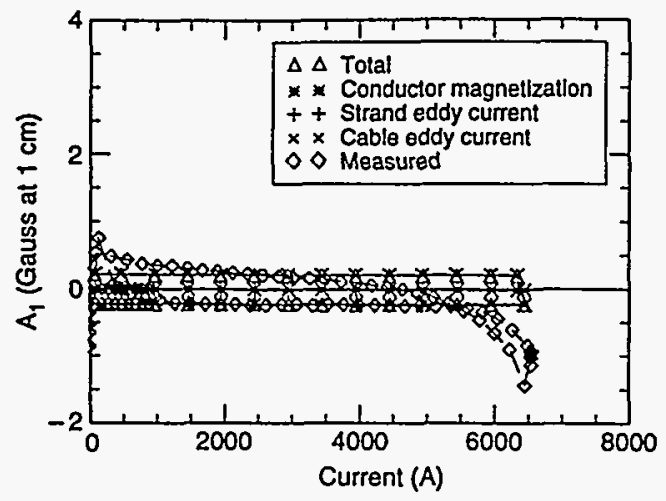

(c)

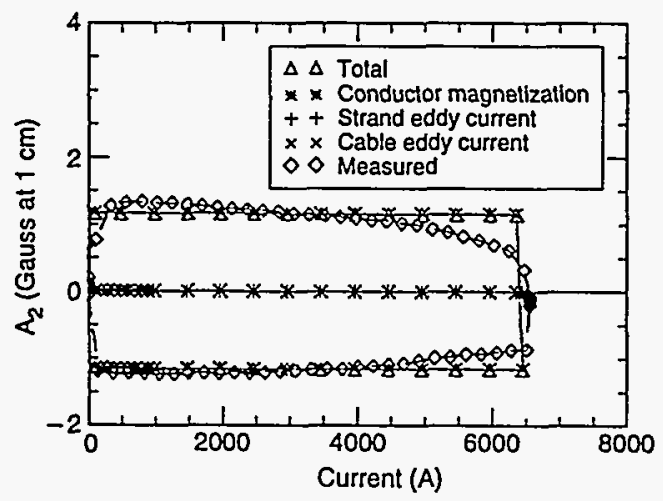

(e)

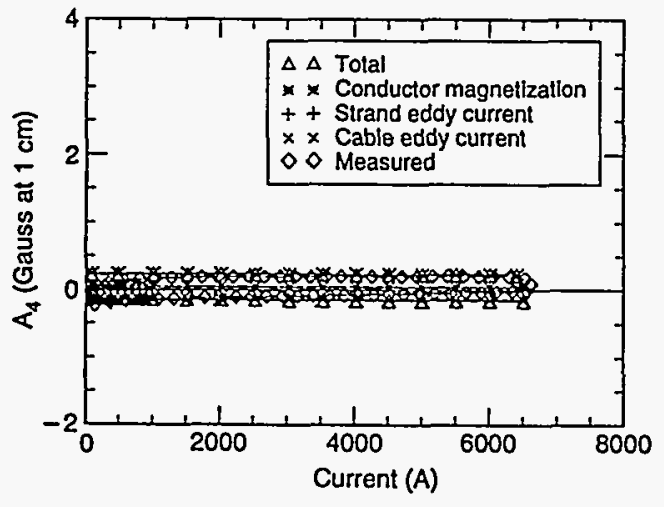

(b)

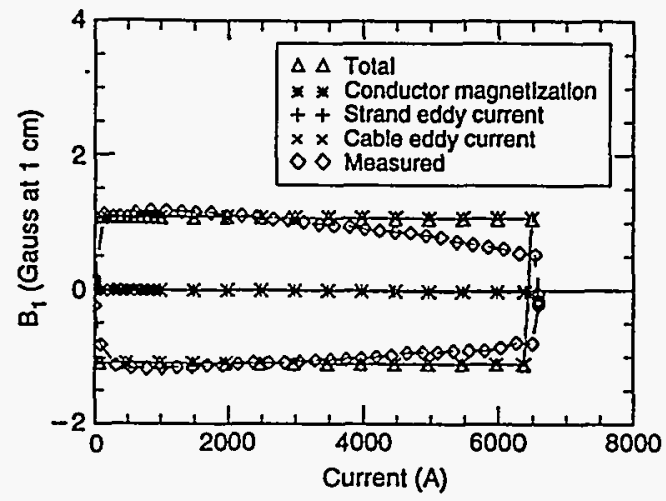

(d)

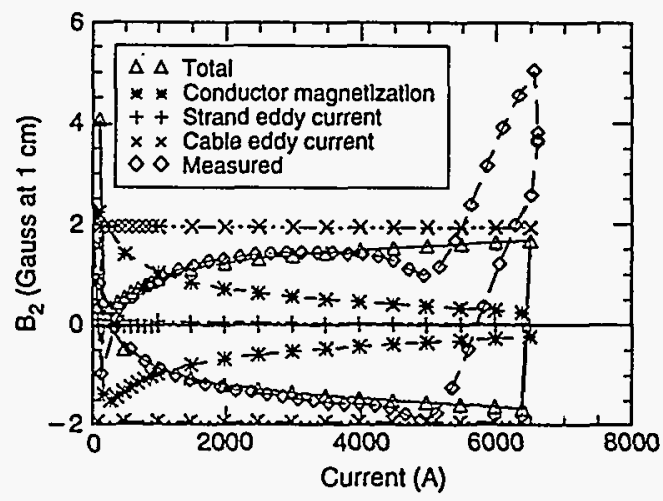

(f)

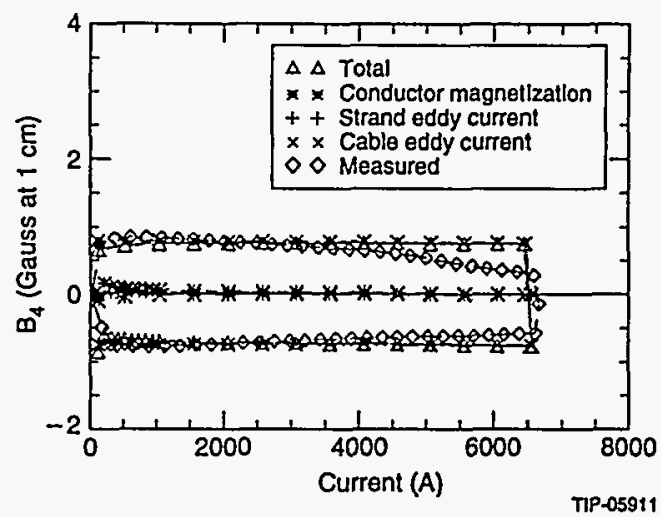

FIGURE 24: Comparison between predicted and measured multipoles fields as a function of current for position 1 of magnet DCA312 at $32 \mathrm{~A} / \mathrm{s}:$ a) skew quadrupole field, b) normal quadrupole field, c) skew sextupole field, d) normal sextupole field, e) skew decapole field, and f) normal decapole field. The measured values are in Gauss and correspond to the rescaled multipole fields at the given position. The computed values are also in Gauss and correspond to the sums of the predicted effects of the persistent magnetization currents and of the intra-strand and inter-strand eddy currents. 
Table VIII summarizes the estimated azimuthal localizations of the inner coil turns with the lowest crossover resistance for the three axial positions along magnet DCA312. Also listed in Tab. VIII are the corresponding values of crossover resistances, inner edge branch currents, and power dissipation per turn unit length for a ramp rate of $1 \mathrm{~A} / \mathrm{s}$. (Note that in the 5-cm aperture SSC cross-section, inner coil turns 6 and 7 are separated by a wedge, and that the magnetic flux embraced by turn 6 , which is closer to the midplane and more perpendicular to the field, is somewhat larger than the magnetic flux embraced by turn 7. Hence, for similar values of crossover resistance-as is the case for the computed crossover resistance distributions at the three axial positions along magnet DCA312-the eddy currents and the power generated in turn 6 are larger than in turn 7.)

TABLE VIII: Estimated localizations and estimated amplitudes of the minima of crossover resistance and of the maxima of eddy current and power dissipation at three axial positions along magnet DCA312.

\section{Position 1 Position 2 Position 3 \\ $5.5 \mathrm{~m}(\mathrm{LE}) \quad 0.5 \mathrm{~m}$ (NLE) $\quad 5.5 \mathrm{~m}$ (NLE)}

Turn Number (Quadrant)

$6 / 7(\mathrm{Q} 1)$

7 (Q2)

7 (Q2)

X-Over Res. $(\mathrm{m} \Omega)$

2.9

2.8

2.0

Peak Branch Curent (A)a

$0.37 / 0.29$

0.30

0.42

Power Dissipation $(\mathrm{mW} / \mathrm{m})^{\mathrm{a}}$

$20 / 12$

13

18

\footnotetext{
${ }^{a}$ At $1 \mathrm{~A} / \mathrm{s}$.
} 
The data in Tab. VIII show that for position 1, the turns with the lowest crossover resistances are turns 6 and 7 of quadrant 1 . This axial position $(5.5 \mathrm{~m}$ from the magnet center towards the lead end) coincides with the axial localization of the $100-\mathrm{A} / \mathrm{s}$ quenches, which, as can be seen in Tab. II, were estimated to have originated between turns 3 and 11 of quadrant 1 . The azimuthal localization of the $100-\mathrm{A} / \mathrm{s}$ quenches is thus consistent with the azimuthal localization of the minima of crossover resistance at the given axial position. Similarly, the data in Tab. VIII show that for position 3, which corresponds to the axial localization of the $150-\mathrm{A} / \mathrm{s}$ quenches $(5.5 \mathrm{~m}$ from the magnet center towards the non-lead end), the turn with the lowest crossover resistance is turn 7 of quadrant 2 . This azimuthal localization is consistent with the estimated azimuthal localizations of the $150-\mathrm{A} / \mathrm{s}$ quenches (between turns 6 and 12 of quadrant 2), and with the azimuthal localizations of the 200-A/s quenches (between turns 6 and 10 of quadrant 2), which also originated in the same area.

Before going further, let us comment on the fact that, according to the data in Tabs. IV and VII, between positions 1 and 3 of magnet DIA312, the quenches and the localization of the minima of crossover resistance shift from quadrant 1 to quadrant 2 . This can be understood qualitatively by considering that the values of $S_{\mathrm{B}_{1}}$ measured at these two positions are of opposite signs (see Tab. IV). As we have explained, a normal quadrupole field arises from left/right asymmetries in the eddy currents. Hence, a change in the sign of the quadrupole field indicates that, at one position, the eddy currents are larger in one side of the coil assembly (e.g., the right side at position 1), while, at the other position, they are larger in the other side of the coil assembly (e.g., the left side at position 3). In our 
simulation, such a shift in the localization of the peak of eddy currents is explained by a shift in the localization of the minima of crossover resistance. It is also reasonable to speculate that this shift is the cause of the shift in quench start localization. Note, however, that although we may have a qualitative understanding of why the quenches moved from quadrant 1 to quadrant 2, we cannot explain the change in axial localization.

For all the cases where the ramp rate dependence of the multipole fields were measured at, or near, the axial localizations of the high ramp rate quenches listed in Tab. II, it was verified that there was a good agreement between the estimated azimuthal localizations of the quenches determined from the pickup coils' data, and the azimuthal localizations of the minima of crossover resistance determined by the optimization process described above. This provides an independent verification of the soundness of our assumptions and of the simulation process we developed. In addition, the fact that the crossover resistances are not uniform, but vary from turn to turn, was confirmed recently by in-situ measurements performed on sections of collared-coil assembly cut from magnet DCA312.64

\subsection{Discussion}

In summary, the simulation described above can account for the observed dynamic field behavior, the measured eddy current losses, and the localization of the high ramp rate quenches of the anomalous type-A magnets. The origin of the anomalous type-A behavior is thus to be found in low and now uniform crossover resistances distributions. The next questions to ask are: what determine the values of the crossover resistances and how can such non-uniformities arise? 
Unlike the HERA strands, the SSC strands are bare. Hence, the crossover resistances are determined mainly by the thickness of the copper oxide layer that develops (or does not develop) during the various steps of cable manufacturing and magnet assembly. Little is known about the parameters that influence the natural growth of copper oxide and no attempts were made to control it. Also, as we described earlier, the SSC strands use a high purity copper (RRR > 300) and they are not heat-treated after the final drawing. As a result, the RRR of the as-received cables is of the order of 40 (see Tab. I). After winding completion, the coils are cured for a couple of hours at a nominal temperature of $135^{\circ} \mathrm{C}$ and under a nominal pressure of $70 \mathrm{MPa}$. Curing is always accompanied by a strong annealing, and the RRRs of the cured coils (as measured during magnet cold-test) are usually in excess of 100. Furthermore, it is known for a fact that the temperature and pressure distributions during coil curing are not uniform, 65 and that some of the turns, which may also be under stress concentration, are heated more rapidly and for a longer time than the rest of the coil. One can then speculate that these pressure and temperature variations, on the background of a un-annealed cable having a particularly low level of oxidation, may be large enough to produce the non-uniformities in the crossover resistances that we have inferred.

As we mentioned before, one of the type-A magnets (magnet DCA317) exhibited very little ramp rate sensitivity, as well in terms of quench current degradation, as in terms of energy losses and field distortions. The only difference between magnet DCA317 and the other type-A magnets built at FNAL is that it was collared twice, and that the delay between coil curing and final collaring was of the order of 120 days, compared to less than 50 days for the other magnets. The speculation here is that the longer delay between curing 
and collaring, during which the un-compressed coils were exposed to air, may have allowed the growth of a thicker copper oxide layer, thus resulting in larger crossover resistances. In addition, the cyclic constraints applied to the cables as a result of multipole collaring may have severed the micro-bridges that could have been formed at the crossovers between strands during coil curing, thus also resulting in larger crossover resistances. Unfortunately, we do not have any experimental facts to support (or contradict) these speculations.

The last question to ask is: why are the IGC strands more inclined to develop this kind of problems than the OST strands? None of the investigations that have been carried out to answer this question have been successful, and, up to this date, we do not have a convincing explanation for the difference in behavior.

\section{MODEL FOR TYPE-B BEHAVIOR}

From the data presented above, we concluded that eddy currents played little role in the behavior of the type-B magnets, and that the dramatic quench current degradation at low ramp rate was not due to ramp-induced heating. The causes for type- $B$ behavior thus have to be found elsewhere. Over the last couple years, many theories have been developed, and many potential causes have been identified, but the SSC project was canceled before any of them could be validated, either by experience or by inspection. Hence, up to this date, we only have a limited and qualitative understanding of what may be at the origin of the type-B behavior.

As we described earlier, the Rutherford-type conductor used in superconducting particle accelerator magnets consists of a few tens of strands, twisted together, and shaped into a flat, two-layer, slightly keystoned cable. Each cable strand is characterized by a 
$V-I$ curve and a self-inductance. Each strand pair is characterized by a mutual inductance. Also, all the strands are coupled through the crossover resistances and the splice resistances at the coil ends. During energization, the current distributes itself among the cable strands according to this intricate network of resistances and inductances.

Let us start by simplifying the problem and considering the case of a cable with insulated strands. In this case, an idea of how the current distributes itself, and how this distribution is affected by the ramp rate, can be obtained from the two-strand model developed in Reference 66 and reproduced in Fig. 25. When applying a constant voltage, $U$, to this model circuit, the current is shared between the two strands according to the system of equations

$$
\begin{aligned}
& U=L_{1} \frac{\mathrm{d} I_{1}}{\mathrm{~d} t}+M \frac{\mathrm{d} I_{2}}{\mathrm{~d} t}+r_{1} I_{1}, \\
& U=M \frac{\mathrm{d} I_{1}}{\mathrm{~d} t}+L_{2} \frac{\mathrm{d} I_{2}}{\mathrm{~d} t}+r_{2} I_{2},
\end{aligned}
$$

where $I_{1}$ and $I_{2}$ are the strands' currents, $L_{1}$ and $L_{2}$ are the strands' self-inductances, $M$ is their mutual inductance, and $r_{1}$ and $r_{2}$ are the splice resistances.

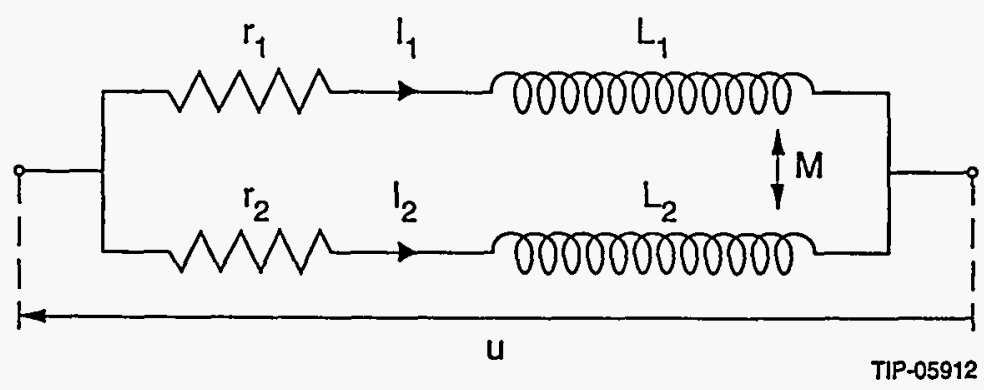

FIGURE 25: Model circuit for two electrically-insulated, magnetically-coupled strands with series resistances. 
This leads to the system of second order differential equations

$$
\begin{aligned}
& \left(L_{1} L_{2}-M^{2}\right) \frac{\mathrm{d} I_{1}^{2}}{\mathrm{~d} t^{2}}+\left(r_{1} L_{2}+r_{2} L_{1}\right) \frac{\mathrm{d} I_{1}}{\mathrm{~d} t}+r_{1} r_{2} I_{1}-r_{2} U=0, \\
& \left(L_{1} L_{2}-M^{2}\right) \frac{\mathrm{d} I_{2}^{2}}{\mathrm{~d} t^{2}}+\left(r_{1} L_{2}+r_{2} L_{1}\right) \frac{\mathrm{d} I_{2}}{\mathrm{~d} t}+r_{1} r_{2} I_{2}-r_{1} U=0 .
\end{aligned}
$$

The general solutions of the system of Eqs. (98) are

$$
\begin{aligned}
& I_{1}(t)=\frac{U}{r_{1}}+I_{1,+} \exp \left(-\frac{\mathrm{t}}{\tau_{+}}\right)+I_{1,-} \exp \left(-\frac{\mathrm{t}}{\tau_{-}}\right), \\
& I_{2}(t)=\frac{U}{r_{2}}+I_{2,+} \exp \left(-\frac{\mathrm{t}}{\tau_{+}}\right)+I_{2,-} \exp \left(-\frac{\mathrm{t}}{\tau_{-}}\right),
\end{aligned}
$$

where $I_{1,+}, I_{1,-}, I_{2,+}$, and $I_{2,-}$ are four integration constants to be determined from the initial conditions, and $\tau_{+}$and $\tau_{-}$are two time constants given by

$$
\begin{aligned}
& \frac{1}{\tau_{+}}=\frac{\left(r_{1} L_{2}+r_{2} L_{1}\right)+\sqrt{\left(r_{1} L_{2}-r_{2} L_{1}\right)^{2}+4 r_{1} r_{2} M^{2}}}{2\left(L_{1} L_{2}-M^{2}\right)}, \\
& \frac{1}{\tau_{-}}=\frac{\left(r_{1} L_{2}+r_{2} L_{1}\right)-\sqrt{\left(r_{1} L_{2}-r_{2} L_{1}\right)^{2}+4 r_{1} r_{2} M^{2}}}{2\left(L_{1} L_{2}-M^{2}\right)} .
\end{aligned}
$$

If the strands' initial currents are zero $\left(I_{1}(t=0)=I_{2}(t=0)=0\right)$, Eqs. (97) show that

$$
\begin{aligned}
& \frac{\mathrm{d} I_{1}(t=0)}{\mathrm{d} t}=\frac{L_{2}-M}{L_{1} L_{2}-M^{2}} U, \\
& \frac{\mathrm{d} I_{2}(t=0)}{\mathrm{d} t}=\frac{L_{1}-M}{L_{1} L_{2}-M^{2}} U .
\end{aligned}
$$


Hence, it appears that the initial rates of current increase are determined by the inductive elements of the circuit.

Furthermore, for $t \gg \tau_{-}$, Eqs. (99) show that

$$
I_{1} \approx \frac{U}{r_{1}},
$$

and

$$
I_{2} \approx \frac{U}{r_{2}} \text {. }
$$

Hence it appears that, for ramps of long duration, the current repartition among the cable strands is determined by the resistive elements of the circuit.

For the sake of the argument, let us now assume that $r_{1} \approx r_{2}$, and that $\left(L_{2}-M\right)$ is larger than $\left(L_{1}-M\right)$. Let us also assume that a quench only occurs when the current in at least one of the strands is larger than a given value, designated as $I_{\mathrm{q}}$. With these assumptions, Eqs. (101) show that, initially, the current in strand 1 increases more rapidly than in strand 2. However, for rates such that the duration of the ramp exceeds $\tau_{-}$, the current has time to redistribute itself through the splice resistances and Eqs. (102) show that it becomes uniform. Then, both strands reach $I_{\mathrm{q}}$ at the same time, and the total transport current, $I_{\mathrm{t}}=I_{1}+I_{2}$, at the time of the quench is

$$
I_{\mathrm{t}} \approx 2 I_{\mathrm{q}}, \quad \text { at low ramp rates. }
$$

For large ramp rates, the current does not have the time to redistribute itself, and strand 1 reaches $I_{\mathrm{q}}$ first. Asymptotes to $I_{1}$ and $I_{2}$ at large ramp rates can be determined by 
assuming that the currents increase linearly as a function of time, with the slopes given by Eqs. (101). Hence, the time $t_{\mathrm{q}}$ at which $I_{1}$ reaches $I_{\mathrm{q}}$ is

$$
t_{\mathrm{q}} \approx \frac{L_{1} L_{2}-M^{2}}{L_{2}-M} \frac{I_{\mathrm{q}}}{U}
$$

and the total transport current at $t_{\mathrm{q}}$ is

$$
I_{\mathrm{t}} \approx\left(1+\frac{L_{1}-M}{L_{2}-M}\right) I_{\mathrm{q}}<2 I_{\mathrm{q}}, \quad \text { at large ramp rates. }
$$

For intermediate ramp rates, the total quench current can be shown to drop from the value given by Eq. (103) to the value given by Eq. (105), following a curve resembling that of the type-B magnets.

Let us now go back to the case of a cable with non-insulated strands. There, the crossover resistances act as parallel paths through which, in addition to the splice resistances, the current redistributes itself. Attempts to describe such a cable lead rapidly to awkward equations, 66 which ${ }^{\circ}$ can be made even more cumbersome by taking into consideration the non-linear $V-I$ characteristics of the strands. ${ }^{67}$ However, common sense tells that the underlying physics remains the same: at large ramp rates, the current repartition is mainly determined by the inductive elements of the circuit, while the static current repartition is only determined by the resistive elements. If the strands are identical and interchangeable, they all carry the same current, and changing the ramp rate is not expected to have any influence. However, if for one reason or another, the strands are not identical or are not interchangeable, the static and dynamic current repartitions can be different. The speculation is then that, as the ramp rate is increased, the current repartition 
changes from one to the other, leading to the kind of quench behavior observed on the type-B magnets. The exact nature of the strand asymmetries at the origin of the current imbalances has yet to be identified.

\section{CASE OF MIXED TYPE-A AND TYPE-B BEHAVIORS}

As we mentioned earlier, the inner cables wound in the twenty 5-cm aperture, $15-\mathrm{m}$ long SSC dipole magnet prototypes were made with strands purchased from three different manufacturers. So far, we have only discussed the behavior of magnets relying on inner strands coming from IGC and OST. The third inner strand manufacturer was Supercon, Inc. Among the magnets with Supercon inner strands, BNL magnets DCA212 and DCA213, and FNAL magnets DCA322 and DCA323 use strands produced from the same series of billets ( 2700 series). Let us now review the ramp rate sensitivity data of these four magnets.

Figure 26 presents a summary plot of quench current versus ramp rate for magnets DCA212, DCA213, DCA322, and DCA323. (Note that some earlier version of this plot have been published which showed a somewhat erratic quench behavior for some of these magnets. It was later found that all the outlying quenches corresponded to pre-cycled ramps. For the plots in Fig. 26, we have retained only the quenches taken following the sequence: quench, ramp to quench.) The two FNAL magnets exhibit a behavior resembling that of the OST type-B magnets, with a significant decrease of the quench current at low ramp rates (although less pronounced than for most of the OST type-B magnets), followed by a much milder degradation at larger rates. Also, the shift in quench start localization from the inner coil pole turn to the multi-turn section near the inner coil midplane takes place between $4 \mathrm{~A} / \mathrm{s}$ and $16 \mathrm{~A} / \mathrm{s}$, which is similar to what was observed on the OST type-B 
magnets (where the shift occurred between $1 \mathrm{~A} / \mathrm{s}$ and $4 \mathrm{~A} / \mathrm{s}$ ), and is well below what was observed on the IGC type-A magnets (where the shift occurred between $25 \mathrm{~A} / \mathrm{s}$ and $50 \mathrm{~A} / \mathrm{s})$. The low ramp rate behavior of the two BNL magnets is identical to that of the FNAL magnets. At larger rates, however, the quench current degradation is much larger than for magnets DCA322 and DCA323, and is reminiscent of that seen on the IGC type-A magnets.

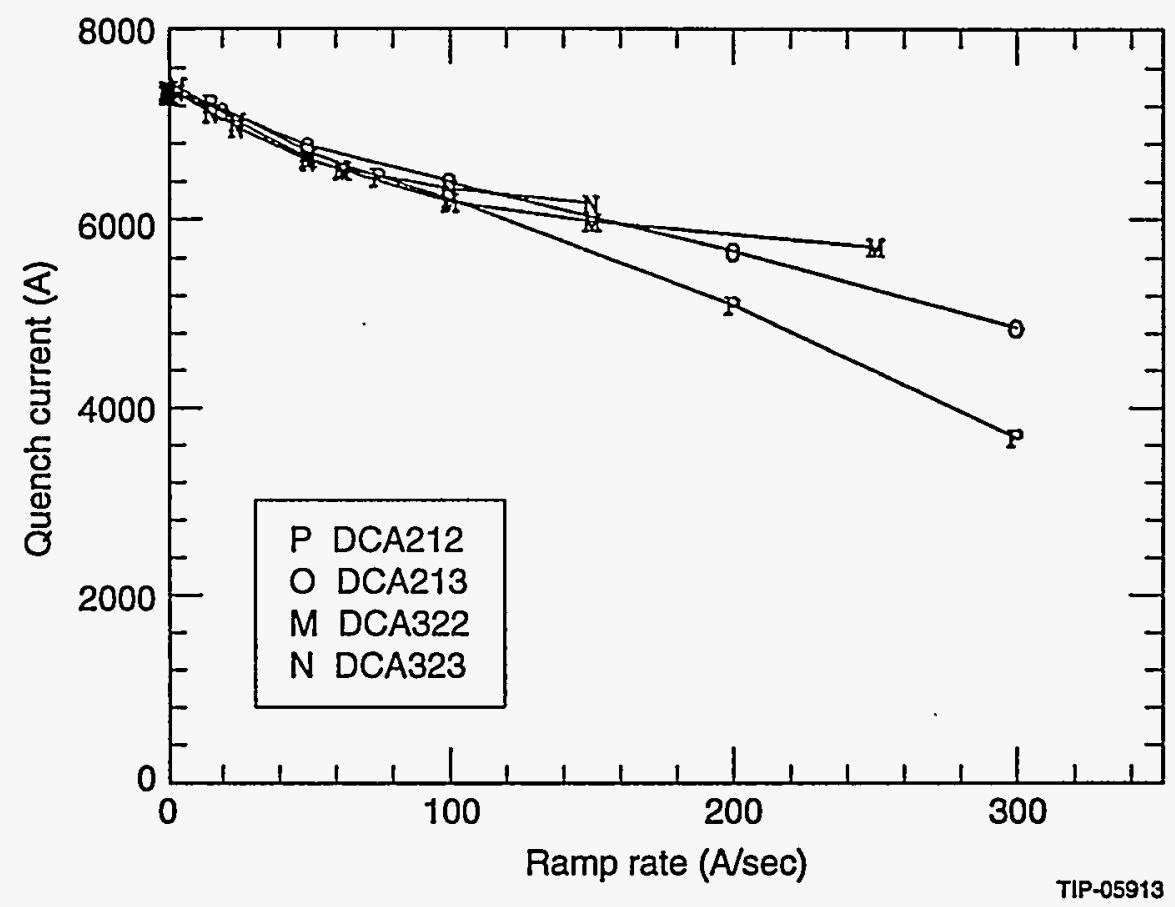

FIGURE 26: Ramp rate sensitivity of selected 5-cm aperture, 15-m long SSC dipole magnet prototypes using inner cable strands manufactured by Supercon (2700-series billets).

Looking now at the energy loss data of Fig. 13 and Tab. V, it appears that, the losses of magnets DCA322 and DCA323 are comparable to the FNAL-measured losses of magnets DCA311, DCA318, and DCA319. Although we rose some questions about the 
reliability of the low-level measurements, we concluded that these numbers could nevertheless be used for qualitative cross-magnet comparison. As such, the behavior of magnets DCA322 and DCA323 is indistinguishable from the behavior of the OST type-B magnets. At BNL, energy loss measurements were only performed on magnet DCA213, and the losses measured on this magnet appear unmistakably larger than the losses measured on magnets DCA211, DCA317, and DCA318. As for the quench performance, magnet DCA213 thus differentiates itself from the regular type-B magnets, and exhibits a behavior reminiscent of the type-A magnets.

In addition, it is worth mentioning that magnetic measurements as a function of ramp rate were performed on magnets DCA213 and DCA323 and that neither magnet exhibited anomalous dynamic field behaviors.

In summary, FNAL magnets DCA322 and DCA323 appear to behave like type-B magnets, while BNL magnets DCA212 and DCA213 present signs of mixed type-A and type-B behaviors: the low ramp rate behavior is similar to that of magnets DCA322 and DCA323, while the behavior at larger rates is reminiscent of the type-A magnets. One known difference between magnets DCA212 and DCA213, on one hand, and magnets DCA322 and DCA323, on the other hand, is the conductor insulation scheme and the associated coil curing cycle. The two BNL magnets rely on an all-Kapton ${ }^{\circledR}$ insulation scheme with a polyimide adhesive coating which requires a short step at $225^{\circ} \mathrm{C}$ in the cure cycle. The two FNAL magnets also use an all-polyimide insulation scheme, but the curing temperature was only $170^{\circ} \mathrm{C}$. As we described earlier, coil curing is always accompanied by a strong annealing of the conductor copper, and one expects the extend of this annealing 
to depend on the curing temperature. Table I(c) shows that the RRRs measured on magnets DCA322 and DCA323 are in the range 60 to 70 , while that measured on magnets DCA212 and DCA213 are of the order of 140. It thus appears that the two magnets with the higher peak curing temperature experienced a much stronger annealing. One may then speculate that this stronger annealing limited the growth of the copper oxide layer at the strand periphery, resulting in smaller crossover resistances, and causing magnets DCA212 and DCA213 to start exhibiting signs of type-A behavior.

\section{CONCLUSION}

The investigations reported here show that the type- $\mathrm{A}$ behavior can be explained in terms of inter-strand eddy currents arising from low and non-uniform resistances at the crossovers between the strands of the two-layer, Rutherford-type cable. They also suggest that anomalies in the transport-current repartition among the cable strands may be the cause of the type-B behavior. However, the SSC project was cancelled before the exact nature of these anomalies could be determined. It is nevertheless the hope of the authors that the experience and knowledge gained during the SSC magnet R\&D program will not get lost and that some of the work presented in this paper can be of use for other programs. 


\section{ACKNOWLEDGEMENTS}

The authors wish to acknowledge the contributions of A.A. Akhmetov, A.E. Badea, T. Bush, D.W. Capone, II, M. Coles, R. Coombes, J. DiMarco, G. Ganetis, M. Garber, C. Goodzeit, A.K. Ghosh, R. Gupta, A. Jain, K. Kim, V.T. Kovachev, J. Krzywinski, J. Kuzminski, P.O. Mazur, R.G. Mints, G. Morgan, J. Muratore, W. Nah, D. Orris, J. Ozelis, E.G. Pewitt, P. Radusewicz, W.B. Sampson, R. Scanlan, R.I. Schermer, G. Snitchler, R. Stiening, J.B. Strait, R. Thomas, J.C. Tompkins, M. Wake, P. Wanderer, E. Willen, Y. Yu, Y. Zhao, and H. Zheng. The authors are grateful to Y. Kimura, T. Shintomi, and K. Tsuchiya for their support in the completion of this work. They also wish to thank C. Swenson for many fruitful discussions. 


\section{REFERENCES}

1. J.R. Sanford and D.M. Matthews, eds., "Site Specific Conceptual Design of the Superconducting Super Collider," SSCL-SR-1056, (1990).

2. W. Burgett, M. Christianson, et al., "Full-Power Test of a String of Magnets Comprising a Half-Cell of the Superconducting Super Collider," Part. Accel., 43, pp. 41-75 (1993).

3. A. Devred, T. Bush, et al., "Review of SSC Dipole Magnet Mechanics and Quench Performance," Supercollider 4, pp. 113-135 (1992).

4. J. Strait, D. Orris, et al., "Quench Performance of Fermilab/General Dynamics Built Full Length SSC Collider Dipole Magnets," Supercollider 4, pp. 365-372 (1992).

5. M. Anerella, J. Cottingham, et al., "Construction and Test Results from 15-m Long, 50-mm Aperture SSC Collider Dipole Models," Supercollider 4, pp. 535-549 (1992).

6. W. Nah, A. Akhmetov, et al., "Quench Characteristics of 5-cm Aperture, 15-m Long SSC Dipole Magnet Prototypes," IEEE Trans. Applied Superconductivity, 3(1), pp. 658-661 (1993).

7. J. Kuzminski, T. Bush, at al., "Quench Performance of 50-mm Aperture, 15-m Long SSC Dipole Magnets Built at Fermilab," Int. J. Mod. Phys. A (proc. Suppl.) 2B, pp. 588-591 (1993).

8. P. Wanderer, M. Anerella, et al., "A Summary of SSC Dipole Magnet Field Quality Measurements," Supercollider 4, pp. 137-149 (1992).

9. S. Delchamps, M. Bleadon, et al., "Magnetic Field Measurements of Fermilab/General Dynamics Built Full Scale SSC Collider Dipole Magnets," Supercollider 4, pp. 251258 (1992). 
10. P. Wanderer, M. Anerella, et al., "Magnetic Design and Field Quality Measurements of Full Length 50-mm Aperture SSC Model Dipoles Built at BNL," Int. J. Mod. Phys. A (proc. Suppl.) 2B, pp. 641-643 (1993).

11. J. Strait, R. Bossert, et al., "Magnetic Field Measurements of Full Length 50-mm Aperture SSC Dipole Magnets at Fermilab," Int. J. Mod. Phys. A (proc. Suppl.) 2B, pp. 656-658 (1993).

12. T. Ogitsu and A. Devred, "Influence of Azimuthal Coil Size Variations on Magnetic Field Harmonics of Superconducting Particle Accelerator Magnets," Rev. Sci. Instrum., 65(6), pp. 1998-2005 (1994).

13. T. Ogitsu, Y. Zhao, et al., "Influence of Cable Eddy Currents on Magnetic Field Harmonics," KEK-Proc. 92-14, pp. 23-27 (1992).

14. Y. Zhao, A. Akhmetov, et al., "Current Dependence of Harmonic Field Coefficients of 5-cm Aperture, 15-m Long SSC Dipole Magnet Prototypes," IEEE Trans. Applied Superconductivity, 3(1), pp. 674-677 (1993).

15. R.I. Schermer, "Status of Superconducting Magnets for the Superconducting Super Collider," to appear in the Proceedings of the 13th International Conference on Magnet Technology, Victoria, BC, Canada, 20-24 September, 1993.

16. R.C. Gupta, S.A. Kahn, and G.H. Morgan, "SSC 50-mm Dipole Cross Section," Supercollider 3, pp. pp. 587-599 (1991).

17. T. Ogitsu, A. Akhmetov, et al., "Mechanical Performance of 5-cm Aperture, 15-m Long SSC Dipole Magnet Prototypes," IEEE Trans. Applied Superconductivity, 3(1), pp. 686-691 (1993).

18. A. Devred, T. Bush, et al., "About the Mechanics of SSC Dipole Magnet Prototypes," AIP Conference Proceedings, 249(2), pp. 1310-1374 (1992). 
19. R.P. Shutt and M.L. Rehak, "Transverse Cooling in SSC Magnets," Supercollider 1, M. McAshan, ed., pp. 209-207 (1990).

20. D. Christopherson, D. Capone, et al., "SSC $40 \mathrm{~mm}$ Cable Results and $50 \mathrm{~mm}$ Design Discussions," IEEE Trans. Magn., 27(2), pp. 1881-1883 (1991).

21. E. Gregory, "Conventional Wire and Cable Technology," AIP Conference Proceedings, 249(2), pp. 1198-1229 (1992).

22. D. Christopherson, D. W. Capone, et al., "Summary of the Performance of Superconducting Cable Produced for the Accelerator System String Test Program," Supercollider 4, pp. 25-32 (1992).

23. C. Swenson, private communication.

24. T.S. Jafferey, R. Coombes, et al., "Fermilab-Built SSC Collider Dipoles Using Low Temperature Curing Insulation Systems With and Without Glass Tape," Proc. 1993 IEEE Part. Acc. Conf., pp. 2769-2771 (1993).

25. D. Leroy, J. Krzywinski, et al., "Quench Observation in LHC Superconducting One Meter Long Dipole Models by Field Perturbation Measurements," IEEE Trans. Applied Superconductivity, 3(1), pp. 781-784 (1993).

26. T. Ogitsu, A. Devred, et al., "Quench Antenna for Superconducting Particle Accelerator Magnets," to appear in the Proceedings of the 13th International Conference on Magnet Technology, Victoria, BC, Canada, 20-24 September, 1993.

27. G. Ganetis, J. Herrera, et al, "Field Measuring Probe for SSC Magnets," Proc. 1987 IEEE Part. Acc. Conf., pp. 1393-1395 (1987).

28. W.H. Press, S.A. Teukolsky, et al., Numerical Recipes in Fortran: the Art of Scientific Computing, Cambridge University Press, pp. 490-529 (1992). 
29. M. Coles, J. DiMarco, et al., "Magnetic Field Multipole Measurement Accuracy in Superconducting Magnet," presented at the 5th International Industrial Symposium on the Super Collider, San Francisco, CA USA, 6-8 May, 1993.

30. C.P. Bean, "Magnetization of Hard Superconductors," Phys. Rev. Lett., 8(6), pp. 250253 (1962).

31. H. Brück, R. Meinke, et al., " Field Distortions from Persistent Currents in the Superconducting HERA Magnets," Particles and Fields, 44, pp. 385-392 (1989).

32. H. Brück, D. Gall, et al., "Persistent Current Effects in the Superconducting HERA Magnets and Correction Coils," Proc. 2nd Eur. Part. Acc. Conf., pp. 1160-1162 (1990).

33. P. Schmüser, "Superconducting Magnets for Particle Accelerators," AIP Conference Proceedings, 249(2), pp. 1099-1158 (1992).

34. R. Gupta, J.G. Cottingham, et al., "A Comparison of Calculations and Measurements of the Field Harmonics as a Function of Current in the SSC Dipole Magnets," Proc. 1991 IEEE Part. Acc. Conf., pp. $42-44$ (1991).

35. R.C. Gupta and A.K. Jain, "Variations in $a_{1}$ Saturation in SSC Collider Dipoles," Proc. 1993 IEEE Part. Acc. Conf., pp. 2778-2780 (1993).

36. H. Brück, D. Gall, et al., "Observation of a Periodic Pattern in the Persistent-Current Fields of the Superconducting HERA Magnets," DESY HERA 91-01 (1991).

37. H. Brück, D. Gall, et al., "Observation of a Periodic Pattern in the Persistent-Current Fields of the Superconducting HERA Magnets," Proc. 1991 IEEE Part. Acc. Conf., pp. 2149-2151 (1991).

38. H. Brück, D. Gall, et al., "Observation of a Periodic Pattern in the Persistent-Current Fields of the Superconducting HERA Magnets," presented at the 12th International Conference on Magnet Technology, Leningrad, USSR, June 23-28, 1991. 
39. A.K. Ghosh, K.E. Robins, and W.B. Sampson, "Axial Variations in the Magnetic Field of Superconducting Dipoles," Supercollider 4, J. Nonte, ed., pp. 765-772 (1992).

40. A.K. Ghosh, K.E. Robins, and W.B. Sampson, "Axial Variations in the Magnetic Field of Superconducting Dipoles and Quadrupoles," Proc. 1993 IEEE Part. Acc. Conf., pp. 2742-2744 (1993)

41. A.K. Ghosh, K.E. Robins, and W.B. Sampson, "The Ramp Rate Dependence of the Sextupole Field in Superconducting Dipoles," to appear in the Proceedings of the 13th International Conference on Magnet Technology, Victoria, BC, Canada, 20-24 September, 1993.

42. R. Stiening, "A Possible Mechanism for Enhanced Persistent Sextupole Decay in SSC Dipoles," SSCL-359 (1991).

43. S. Delchamps, R. Hanft, et al., "AC Loss Measurements of SSC Dipole Magnets," KEK-Proc. 92-14, pp. 19-22 (1992).

44. J.P. Ozelis, S. Delchamps, et al., "AC Loss Measurements of Model and Full Size $50 \mathrm{~mm}$ SSC Collider Dipole Magnet at Fermilab," EEE Trans. Applied Superconductivity, 3(1), pp. 678-681 (1993).

45. R. Thomas, private communication.

46. N.M. Wilson, Superconducting Magnets, Clarendon Press, Oxford, p. $174 \mathrm{ff}$ (1983).

47. R.I. Schermer, private communication.

48. G.B.J. Mulder and E.M.J. Niessen, "Coupling Losses of Multifilamentary Superconductors Having Several Concentric Regions and Mixed Matrix," IEEE Trans. Applied Superconductivity, 3(1), pp. 142-145 (1993).

49. W.J. Carr, Jr., "Conductivity, Permeability, and Dielectric Constant in a Multifilament Superconductor," J. Appl. Phys., 46, pp. 4043-4047 (1975). 
50. W.B. Sampson, M. Garber, and A.K. Ghosh, "Normal State Resistance and Low Temperature Resistance of Superconducting Cables for Accelerator Magnets," IEEE Trans. Magn., 25(2), pp. 2097-2100 (1989).

51. Y.Z. Lei, T. Shintomi, et al., "AC Loss Measurements of Rutherford Type Superconducting Cables under Mechanical Stresses," KEK-Proc. 92-14, pp. 23-27 (1992).

52. G.H. Morgan, "Eddy Currents in Flat Metal Field Superconducting Braids," J. Appl. Phys., 44, pp. 3319-3322 (1976).

53. V. E. Sytnikov and G. G. Svalov, "Coupling losses in superconducting transposed conductors located in changing magnetic field," Cryogenics, 29, p. 926-930 (1989).

54. A.A. Akhmetov, A. Devred, and T. Ogitsu, "Periodicity of Cross-Over Currents in a Rutherford-Type Cable Subjected to a Time-Dependent Magnetic Field," J. Appl. Phys., 75(6), pp. 3176-3183 (1994).

55. G.H. Morgan, private communication.

56. D. Orrell, private communication.

57. F.T. Cole, M.R. Donaldson, et al., eds., "A Report on the Design of the Fermi National Accelerator Laboratory Superconducting Accelerator," FNAL (1979).

58. H. Kaiser, "Design of Superconducting Dipole for HERA," Proceedings of the XIII-th International Conference on High Energy Physics Accelerators, Novosibirsk, USSR, August 7-11, 1986, pp. 49-52.

59. P.A. Thompson, R.C. Gupta, S.A. Kahn, H. Hahn, G.H. Morgan, P.J. Wanderer, and E. Willen, "Revised Cross Section for RHIC Arc Dipole Magnets," Proc. of 1991 IEEE Part. Acc. Conf., pp. 2245-2247 (1991).

60. A.K. Ghosh, private communication. 
61. H. Brück, private communication.

62. W.H. Press, S.A. Teukolsky, et al., Numerical Recipes in Fortran: the Art of Scientific Computing, Cambridge University Press, (1992) p. 423ff.

63. User's Manual of the IMSL ${ }^{\circledR}$ MATH/LIBRARY ${ }^{\text {TM }}$, MALB-USM-UNBND-EN89011.1, pp. 892-894 (1989).

64. V.T. Kovachev, M.J. Neal, et al., "Interstrand Resistance of SSC Magnets," submitted to Cryogenics.

65. R. Sims, private communication.

66. B. Turck, "Influence of a Transverse Conductance on Current Sharing in a Two-Layer Conductor Superconducting Cable," Cryogenics, 448-454 (1974).

67. A. Akhmetov, A. Devred, et al., "Current Loop Decay in Rutherford-Type Cables," to appear in the Proceedings of the 5th Annual International Industrial Symposium on the Super Collider, San Francisco, CA USA, 6-8 May, 1993. 